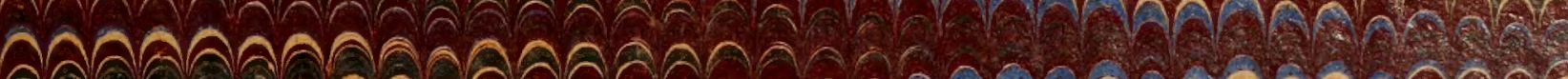

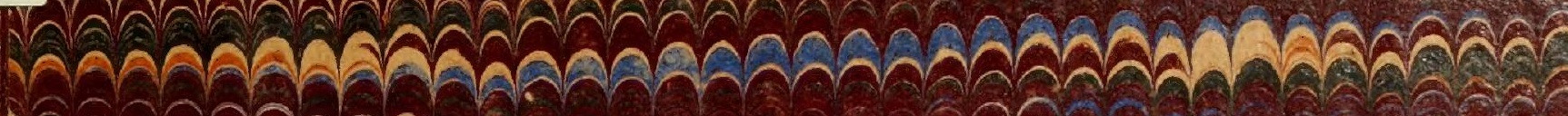
जิ

ลิÁ

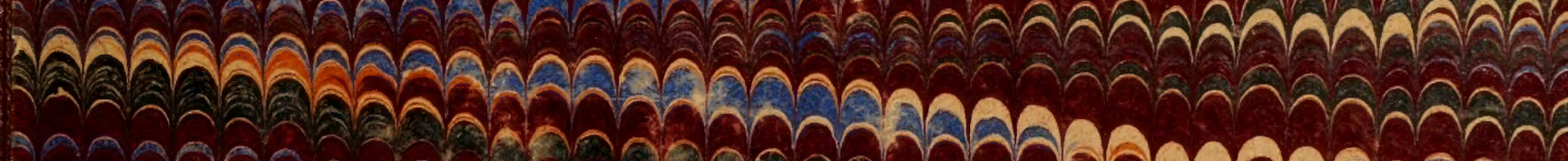

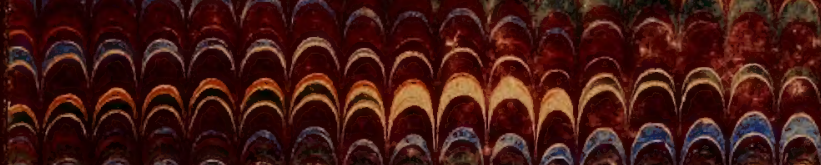

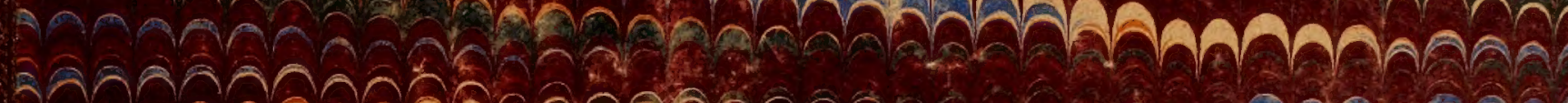
-

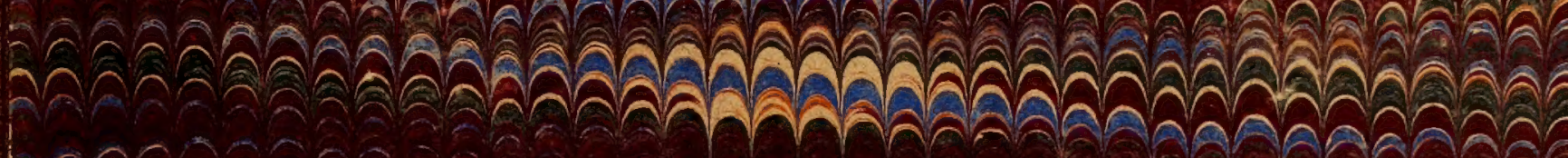
Sิ

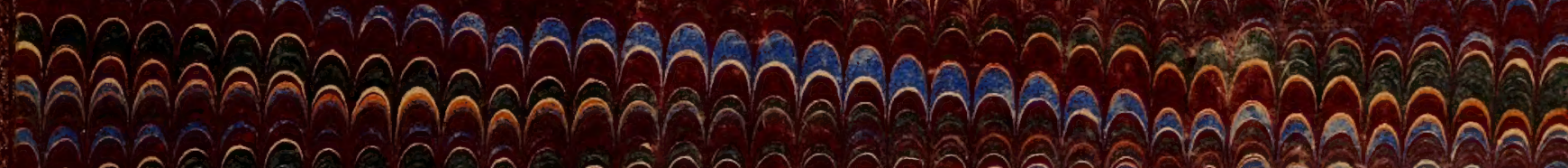

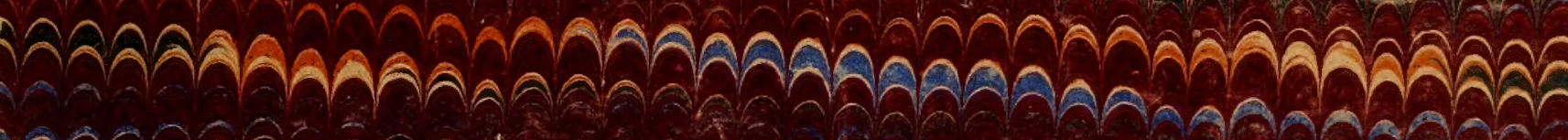

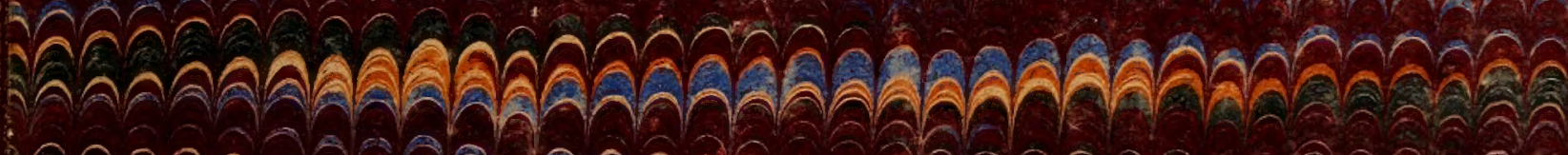
r

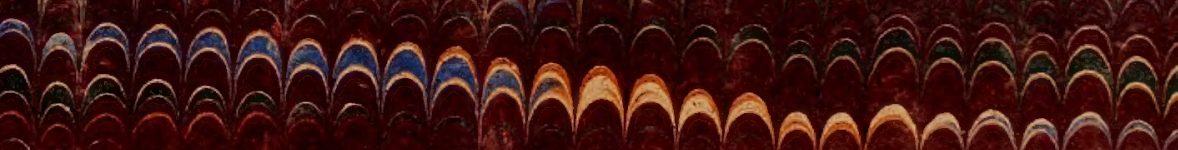

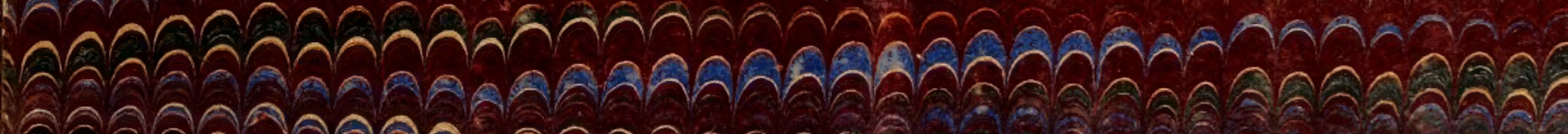
ลลลลลลลลลลดลลลลลว

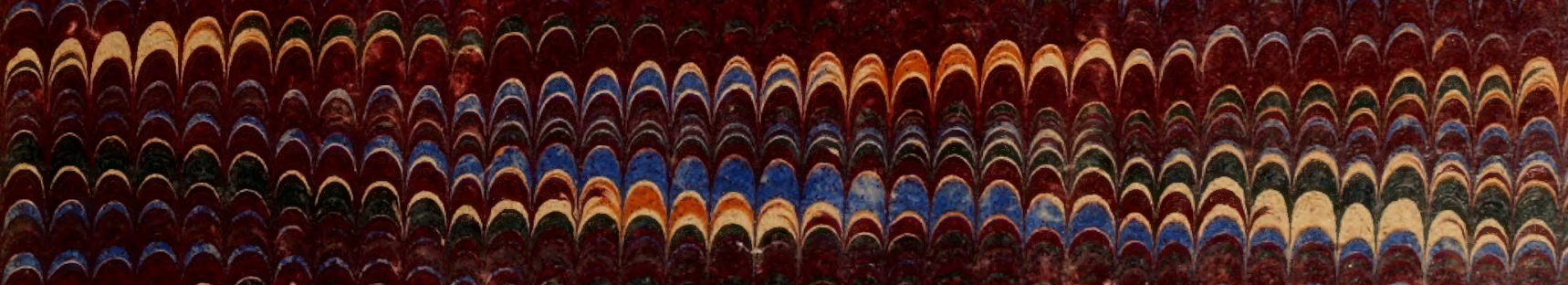

\title{
* * * * * *
}

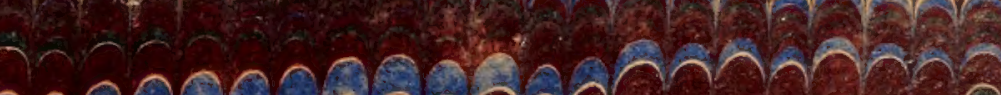
Añ

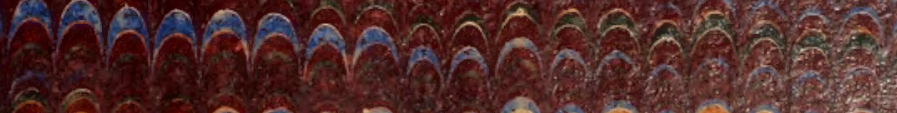

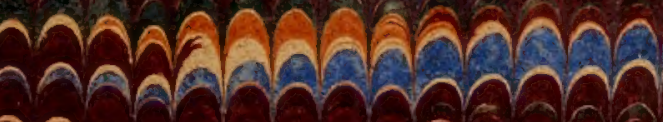

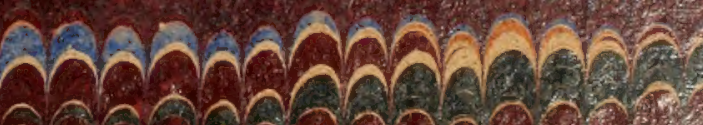

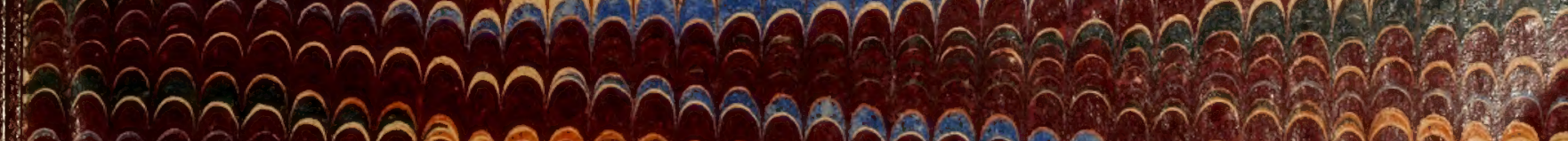




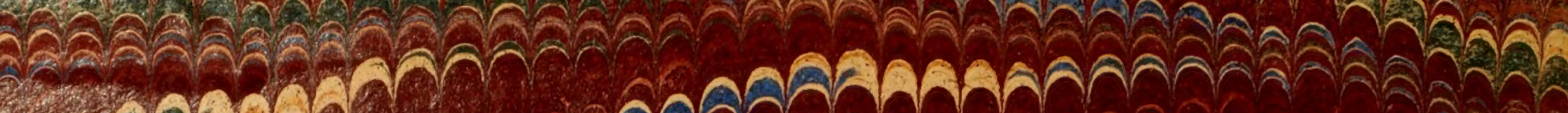

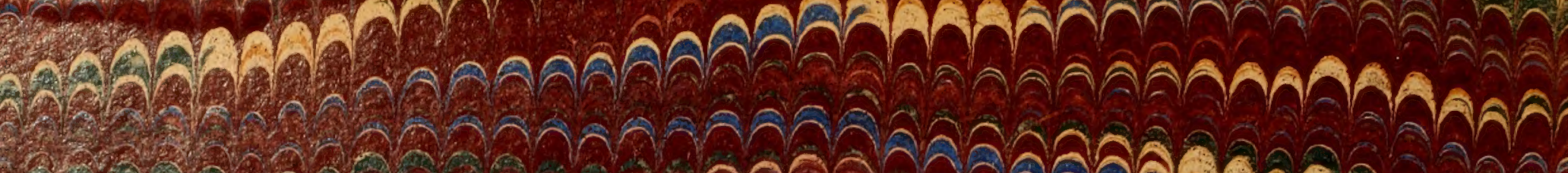
4.

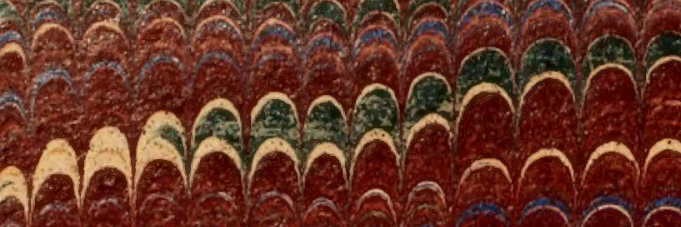

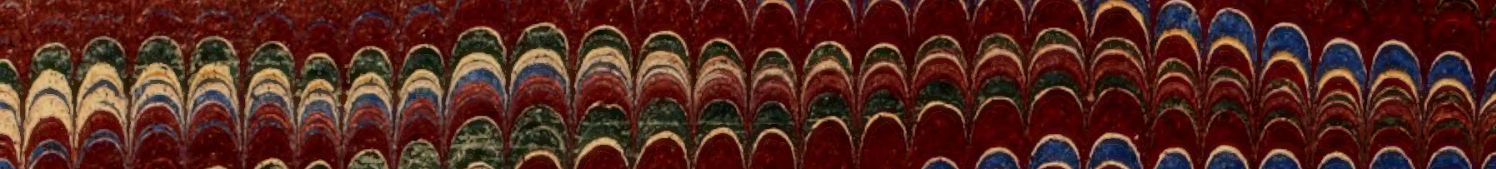

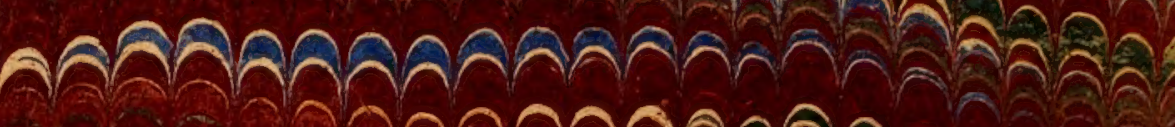
Aงกำกำ

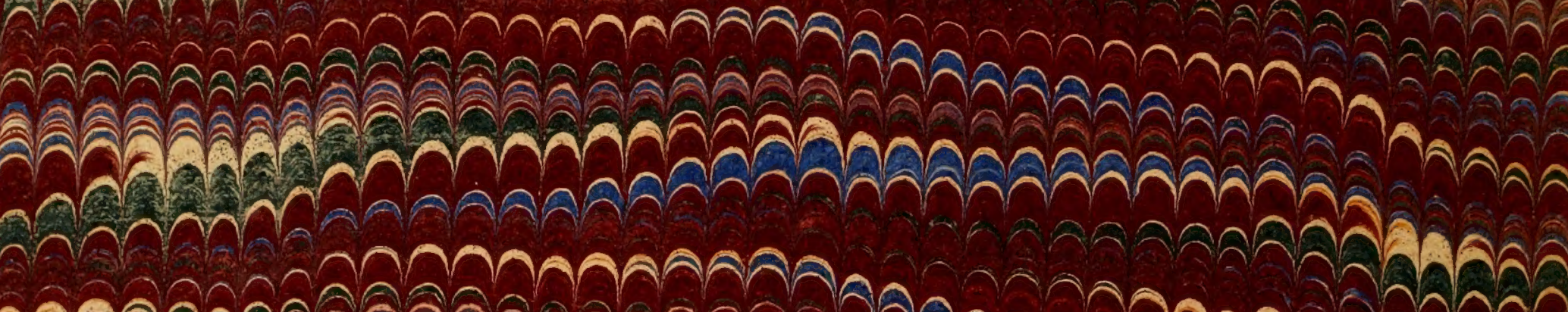

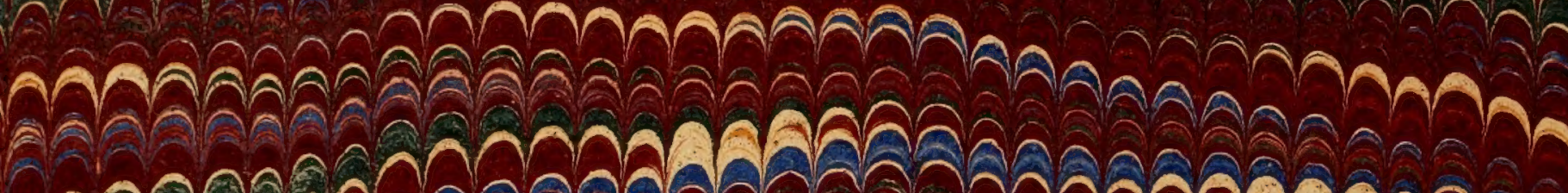

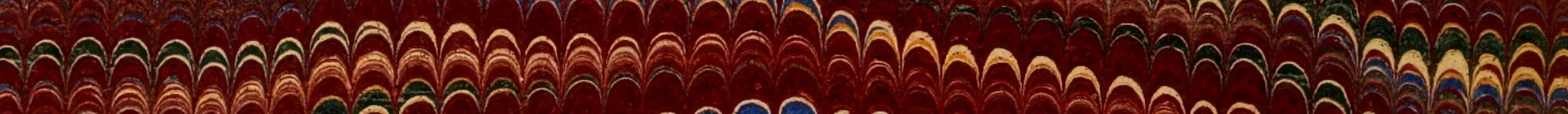

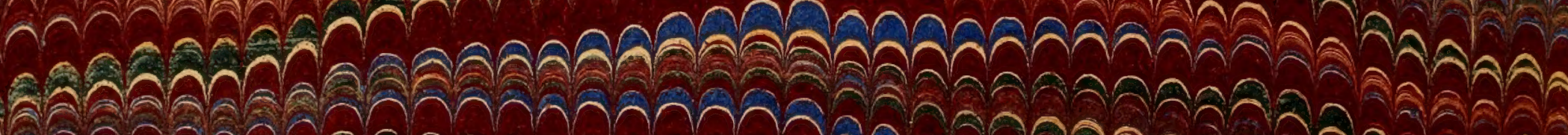

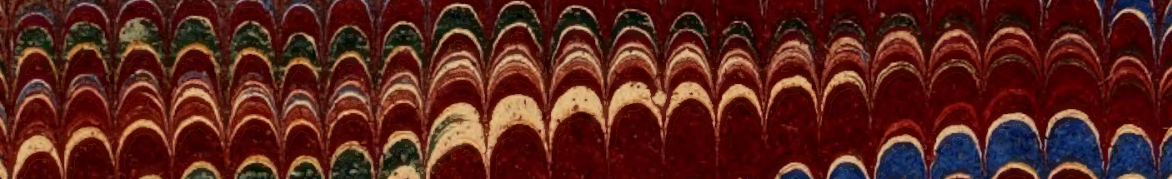

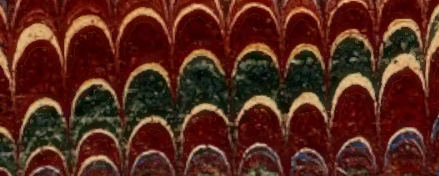

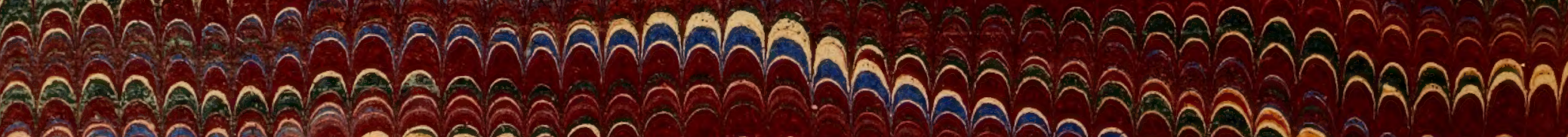

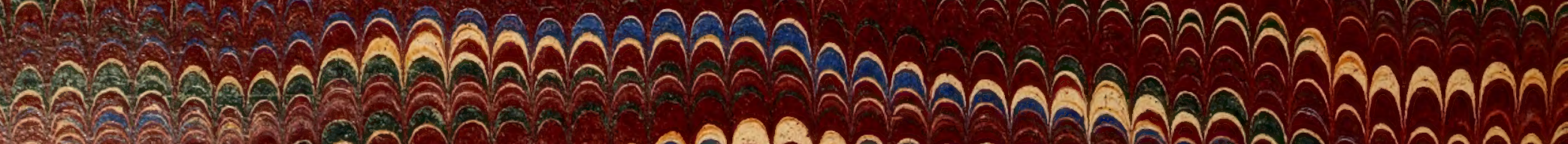

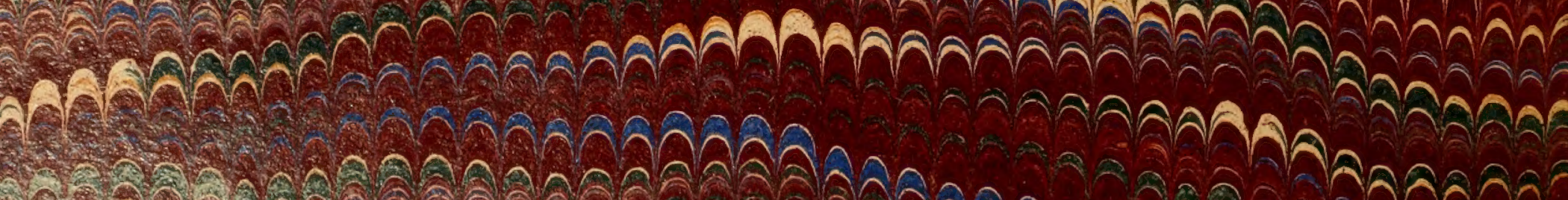

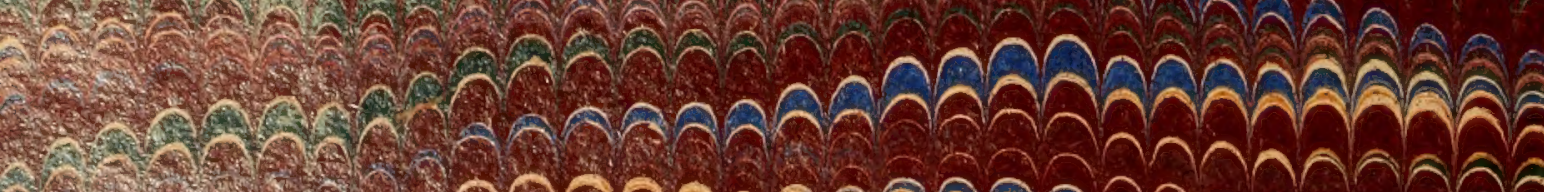
ล:- -

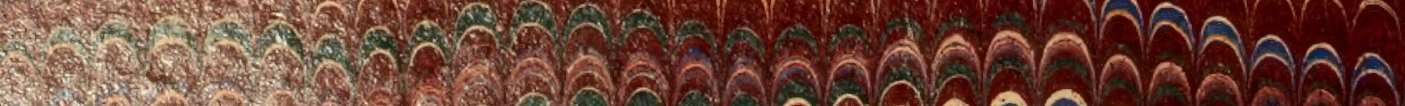




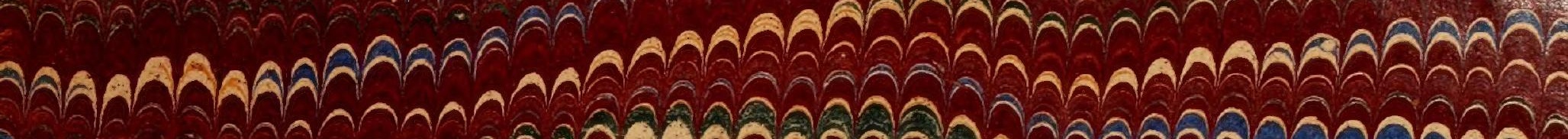

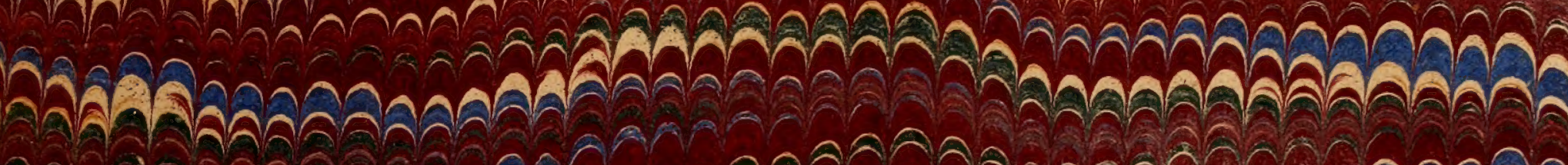
ลลคลลคลล นิน

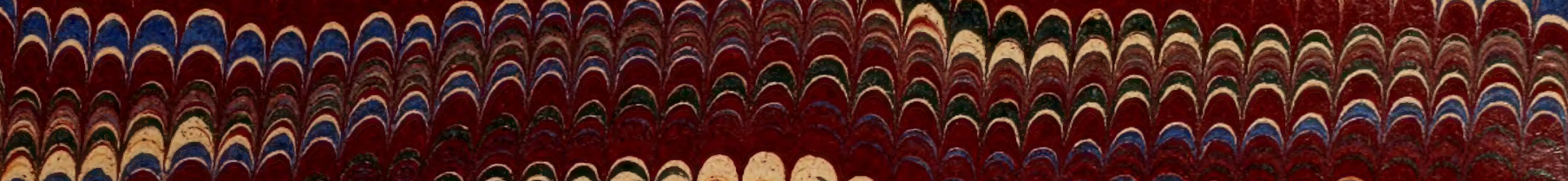

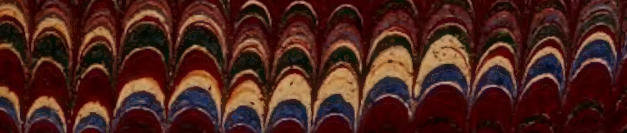

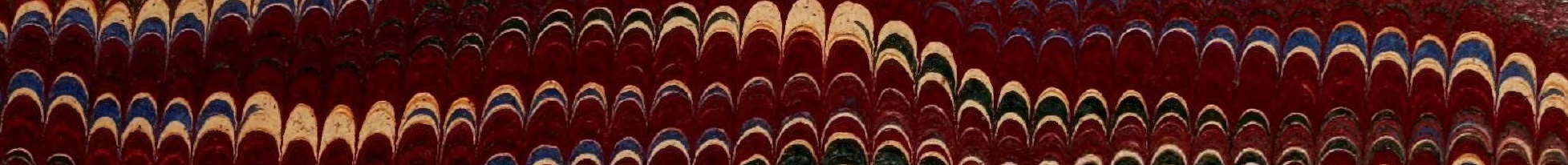

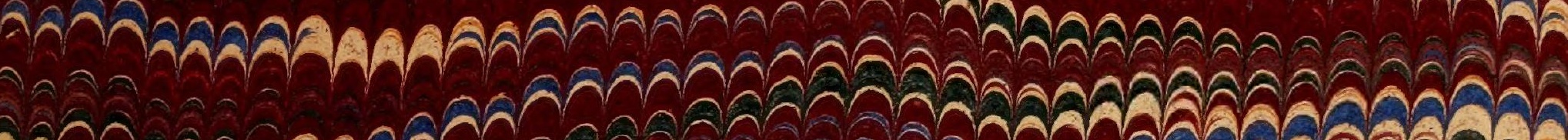

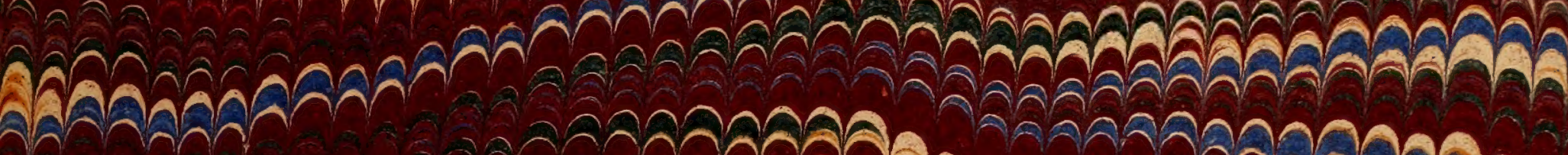

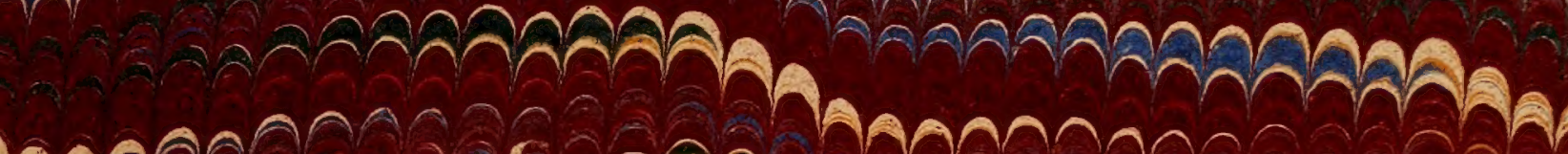

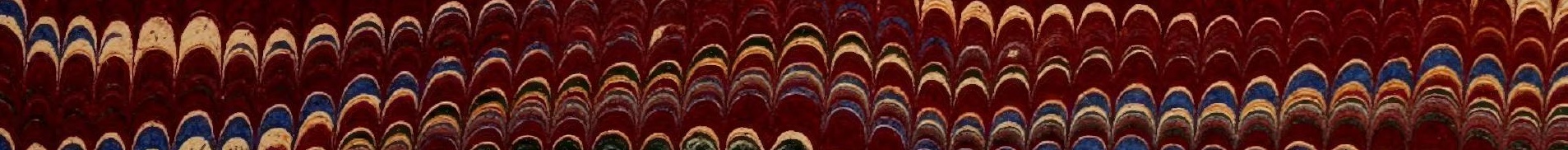

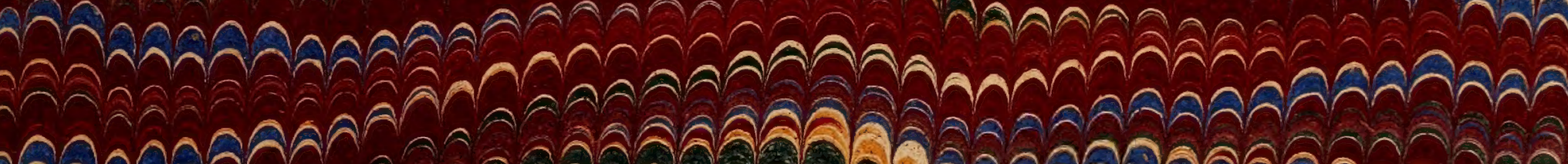

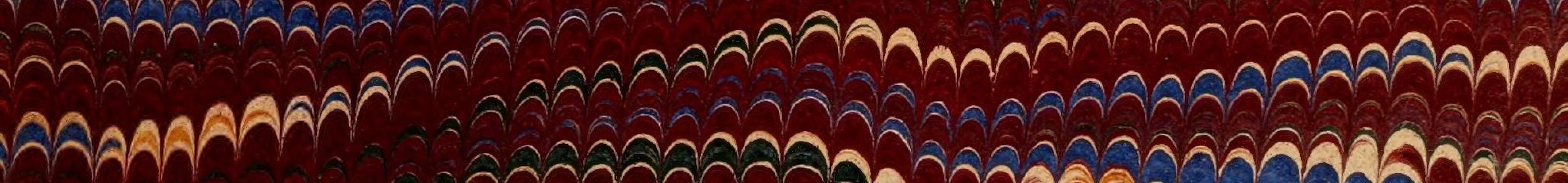
คค

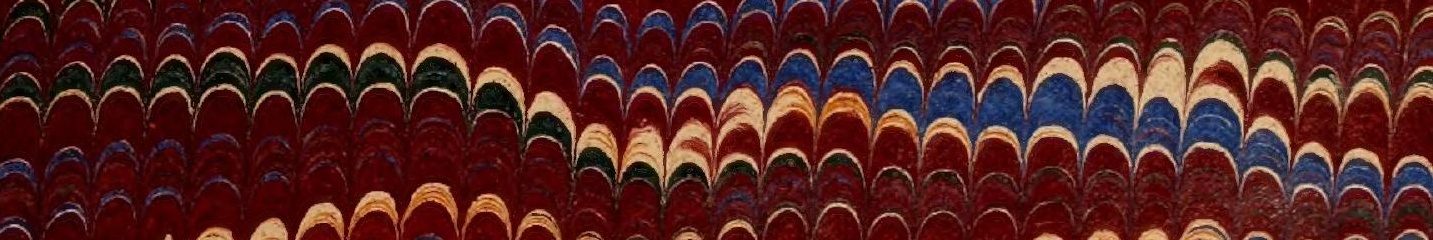

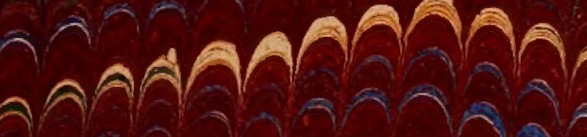

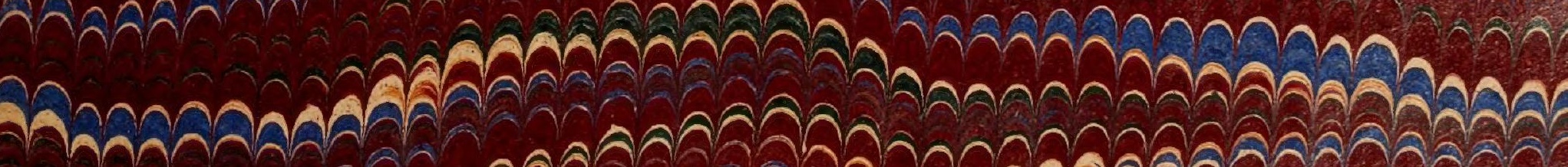
1

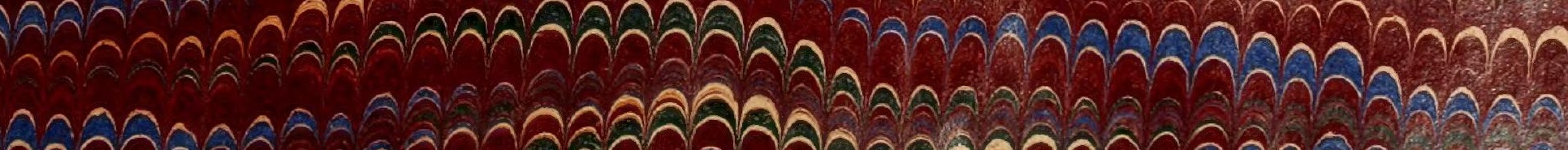

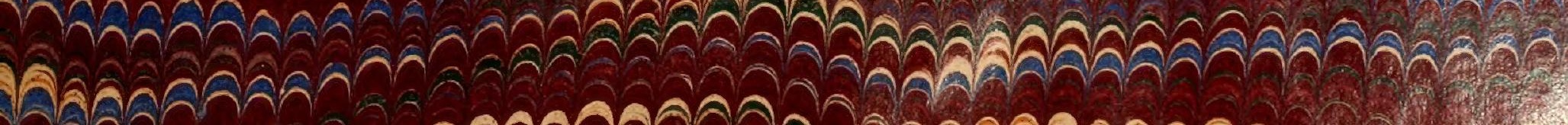

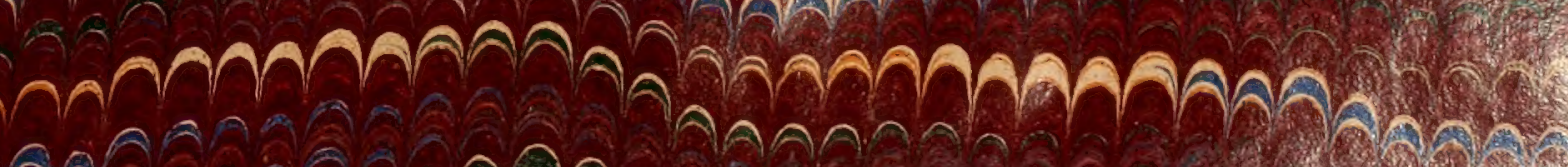









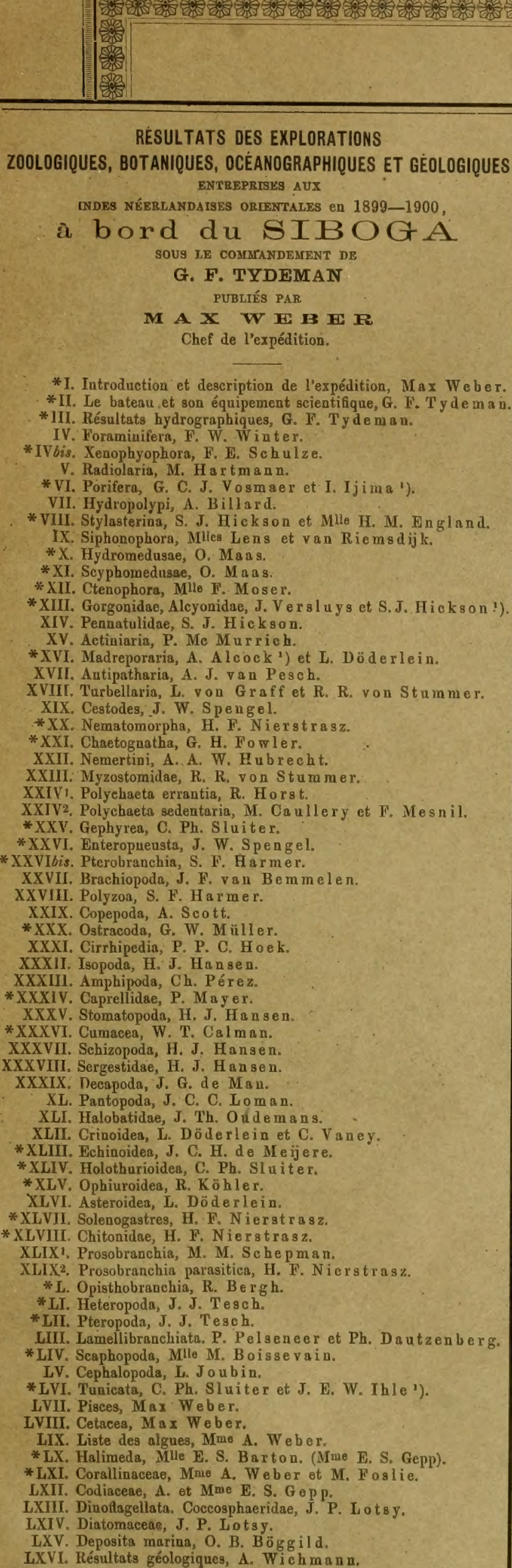

\section{RESULTATS OES EXPLORATIONS} ZOOLOGIQUES, BOTANIQQUES, OCÉANOGRAPHIQUUES ET GËOLOGIQQUES ENTREPRISUS AUX

INDES NÉERLANDATSES ORIENTALES En 1899-1900,

à bord du SIBOG $A$. SOUg T.E COMMRANDEMENT DE

G. F. TYDEMAN pUbLIÉS PAR

M A X W E H E R Chef de l'expédition.

*I. Introduction et description de l'expédition, Max We ber. *II. Le bateau et son équipement scientifique, G. F. Ty de ma in * III. Résultats hydrographiques, G. F. T yde ma n.

IV. Foraminifera, F. W. Winter.

*IVbis. Xenophyophora, F. E. Schulze.

V. Radiolaria, M. Hartmann.

* VI. Porifera, G. C. J. Vosmaer et I. I jima I).

vII. Hydropolypi, A. Billard.

* VIII. Stylasterina, S. J. Hickson et Mlle H. M. England.

IX. Siphonophora, Mliea Lens et van Riemsdijk.

* X. Hydromedusae, O. Ma a s.

* XI. Scyphomedusae, O. M a a s.

*XII. Ctenophora, Mire F. Moser.

* XIII. Gorgonidae, Alcyonidae, J. Ve r sl u ys et S. J. Hi okson!

XIV. Pennatulidae, S. J. Hickson.

XV. Aetiniaria, P. Me Murrieh.

*XVI. Madreporaria, A. A leock ${ }^{1}$ ) et L. Döderlein

XVII. Antipatharia, A. J. van Pesch.

XVIII. Turbellaria, L, von Graff et R. R. von Stummer.

XIX. Cestodes, J. W. S pengel.

*XX. Nematomorpha, H. F. Nierstrasz.

*XXI. Chaetognatha, G. H. Fo w ler.

XXII. Nemertini, A. A. W. Hubrecht.

XXIII: Myzostomidae, $R$, $R$, von Sturamer.

XXIVi. Polyehaeta errantia, R. Horst.

XXIV2. Polychaeta sedentaria, M. Caullery et F. Mesnil.

* XXV. Gephyrea, C. Ph. Sluiter.

* XXVI. Enteropueusta, J. W. Spengel.

*XXVIbis. Pterobranchia, S. F. Harmer.

XXVII. Brachiopoda, J. F. van Bemmelen.

XXVIII. Polyzoa, S. F. Harme r.

XXIX. Copepoda, A. Scott.

*XXX. Ostracoda, G. W. Müller.

XXXI. Cirrhipedia, P. P. C. Hoek.

XXXII. Isopoda, H. J. Han sen.

XXXIII. Amphipoda, Ch. Pérez.

* XXXIV. Caprellidae, P. M a yer.

XXXV. Stomatopoda, H. J. Ha n sen

* XXXVI. Cumacea, W. T. Calman.

XXXVII. Schizopoda, H. J, Hansen.

XXXVIII. Sergestidae, H. J. Hansen.

XXXIX. Decapoda, J. G. de Man.

XI. Pantopoda, J. C. C. Loman.

XLI. Halobatidae, J. Th. O d demans.

XLII. Crinoidea, L. Döderlein et C. Vaney.

* XLIII. Echinoidea, J. C. H. de Meijere.

* XLIV. Holothurioidea, C. Ph. Sluiter.

*XLV. Ophiuroidea, R. Köhler.

XIVI. Asteroidea, L. Döderle in.

*XLVII. Solenogastres, H. F. Nierstrasz.

* XLVIII. Chitonidae, H. F. Nierstrasz.

XLIX1. Prosobranchia, M. M. Schepman.

XIIX2. Prosobranchia parasitica, H. F. Nierstrasz

* L. Opisthobranchia, R. Bergh.

* LI. Heteropoda, J. J. T esch

* LII. Pteropoda, J. J. Tese h.

LIII. Lamellibranchiata. P. Pelseneer et Ph. Dautzen berg

* LIV. Scaphopoda, Mlle M. B oisseva in.

LV. Cephalopods, L. Jou bin.

* LVI. Tunicata, C. Ph. Sluiter et J. E. W. Ihle ').

LVII. Pisces, Max Weber.

LVIII. Cetacea, Mas Weber.

LIX. Liste des algues, Mme A. W e ber.

* LX. Halimeda, Mlle E. S. Barton. (Mrue E. S. Gepp).

* LXI. Corallinaceae, Mrne A. Weber et M. Foslie.

LXII. Codiacere, A. et Mme E. S. G op p.

LXIII. Dinoflagellata. Coccosphaeridae, J., P. L ots y.

LXIV. Diatomaceae, J. P. Lotsy.

LXV. Deposita marina, O. B. Bögrild.

LXVI. Résultats géologiques, A. Wichmann,

\section{Siboga-Expeditie}

\section{DIE ALCYONIDER DER SIBOCAAEXPPDITION}

\section{CORALLIIDE}

BY

SYDNEY J. HICKSON M.A., F.R.S

Professor of Zoology in the Victoria University of Manchester

\section{PSEUDOCLADOCHONUS HICKSONI n. g. a. sp.}

\author{
$D^{R}$. J. VERSLUYS \\ Privat-Docent an đer Universität Amsterdam
}

Mit 3 Tafeln und 16 Figuren im Text

Monographie XIII $c$ aus

\section{UITKOMSTEN OP ZOOLOGISCH, BOTANISCH, OCEANOGRAPHISCH EN GEOLOGISCH GEBIED}

verzameld in Nederlandsch Oost-Indië $1899-1900$

aan boord H. M. Siboga onder commando van

Luitenant ter zee Ie kl. G. F. TYDEMAN

UITGEGEVEN DOOR

Dr. MAX WEBER

Prof. in Amsterdam, Leider dex Expeditie

(met medewerking van de Maatschappij ter bevordering van het Natuurkundig Onderzoek der Nederlandsche Koloniën)

BOEKHANDEL EN DRUKKERIJ

\author{
F. J. BRILL \\ LEIDEN
}
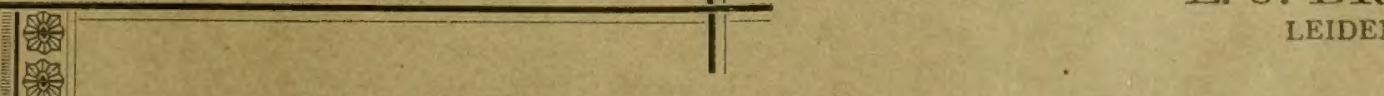
Voor de uitgave van de resultaten der Siboga-Expeditie hebben bijdragen beschikbaar gesteld:

De Maatschappij ter bevordering van het Natuurkundig Onderzoek der Nedeplandsche Koloniën.

Het Ministerie van Koloniën.

Het Ministerie van Binnenlandsche Zaken.

Het Koninklijk Zoologisch Genootschap "Natura Artis Magistra" te Amsterdam.

De ,Oostersche Handel en Reederij" te Amsterdam.

De Heer B. H DE WAAL Oud-Consul-Generaal der Nederlanden te Kaapstad.

M. B. te Amsterdam. 


\section{SIBOGA-EXPEDITIE.}




\section{Siboga-Expeditie}

\section{UITKOMSTEN}

OP

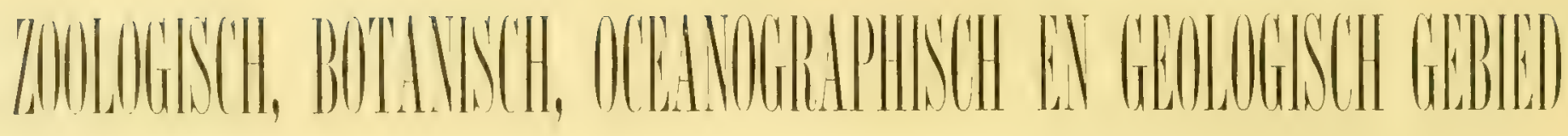

VERZAMELD IN

NEDERLANDSCH OOST-INDIË I899-I900

A AN BOORD H. M. SIBOGA ONDER. COMMANDO VAN

Luitenant ter zee $\mathrm{t}^{\mathrm{e}} \mathrm{kl}$. G. F. TYDEMAN

LITGEGEVEN DOOR

Dr. MAX WEBER

Prof, in Amsterdam, Leider der Expeditie 


\section{Siboga-Expeditie}

XIII.c

\section{DIE ALCÝONIDEN DER SIBOGA-EXPEDITION \\ I. CORALLIIDÆ}

13Y

SYDNEY J. HICKSON iI.A., F.R.S.

II. PSEUDOCLADOCHONUS HICKSONI n. g. n. sp.

VON

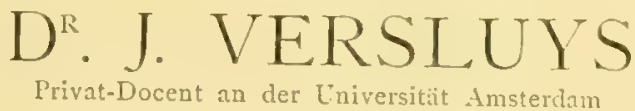

Mit 3 Tafeln und 16 Figuren im Text

BLCHHANDLLNG Z゙ND DRLCKEREI

I. J. I3IIILI

I.EIDEN - I907 



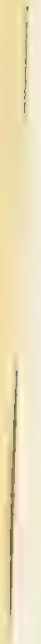




\section{Correction.}

Page I under "Family Coralliidx Gray emend. Ridley" should stand Corallide instead of Corrallidx. 


\title{
DIE ALCYONIDEN DER SIBOGA-EXPEDITION
}

\author{
I. CORALLIID $Æ$
}

BY

SYDNEY J. HICKSON M.A., F.R.S.,

Professor of Zoology in the Victoria University of Manchester.

With one plate.

Family Corallind aray emend. Ridley.

Corrallida Gray, Proc. Zool. Soc. 1857, p. 286.

Corallince Milne Edwards, Hist. Nat. Corall. t. I, p. 20 I.

Corallidice Ridley, Proc. Zool. Soc. IS82, p. $22 \mathrm{I}$.

The family Coralliidx is distinguished from the other families of the sub-order Pseudaxonia by the hard calcareous imperforate axis formed by the growth and adjustment of the spicules of the deeper layers of the coenenchym accompanied by a calcification of the intervening mesogloea. In the Sclerogorgiidae there is also a distinct imperforate axis but the long spindle shaped spicules of which it is built up are surrounded by a sheath of horny substance which renders the axis more flexible than it is in the Coralliidx. In the Briareidxe the axis is porous.

The manner in which the hard axis of Corallizm is formed has been studied by DE LACAzEDuthiers (4), Kölliker (3), Nicholson (6) and Funabashi (3). According to de Lacaze-Duthiers it is formed of two elements, the spicules and the cement, or as it was expressed by KöLIIKER 1. c. p. I47: "Die Axe von Corallium durch die Vereinigung von Kalkkörpern und einer verkalkten Zwischensubstanz entsteht".

When the axis is dissolved slowly in weak acids, according to Fuvabasui, a spongy organic substance is left behind, and when this is examined by the microscope it is found to consist entirely of the empty sacs of spicules, without any other cementing substance between them.

The interpretation of these investigations appears to be that the spicules of the coenenchym, when they have reached their full size and are seated in the inner or deeper layers of 
the soft bark, become cemented together by the cement substance which is at first horny but subsequently becomes calcified and that the important difference, as regards the axis, between the Sclerogorgiide and the Coralliidxe is that in the former family the cement substance does not becomes calcified in the latter it does. The axis of other members of the Pseudaxonia differs from that of these two families in being porous or lacunar ${ }^{1}$ ). As regards the origin of the cement substance we have little or no direct evidence, but the evidence of comparative anatomy suggests very forcibly that it is not formed by the activity of the scleroblasts but by a modification of the chemical character of the mesogloea.

The expression used by WRIGHT and Studer ( 7 ) that "the axis of Sclerogorgiidx is formed of a tissue consisting of numerous closely intercalated elongate spicules with dense horny sheaths" suggests that the cement substance is formed by a fusion of previously distinct sheaths formed by the scleroblasts. The evidence afforded by KöLliker distinctly contradicts this hypothesis, but supports the view that it is formed from mesogloea. How the mesogloea is changed chemically is unknown. The amount of organic matter retained in it when the calcification is completed is very small and the nuclei of mesogloeal cells, if they are present, have hitherto escaped notice. It is clearly incorrect, from these observations, to describe the axis of the Coralliidxe as the "result of spicules fused together".

Dimorphism. The colonies of Coralliidae are dimorphic. Moseley (5) was the first to call attention to the dimorphism of Corallizm nobile a feature of this species which was overlooked or misunderstood by DE LACAZE-Duthiers. Kishinouye (3), has proved that Corallizm japonicum, C. konojoi, C. inutile and C. clatizs are dimorphic. I can also state from the examination of a specimen from off the Irish coast that $C$. jolmstoni is dimorphic. A curious point of difference between Corallizm nobile of the Mediterranean Sea and the other species of the genus is to be found in the fact that the sexual organs are found only in the autozooids of Corallim nobile whereas they are found only in the siphonozooids of those species mentioned above that have been carefully examined. DE LACAZE-DuthiERs' description and figures are conclusive on the first point as regards Corallizm nobile, but I have confirmed them by the examination of a series of sections through a piece of coral from Naples kindly given to me by Dr. Editi Pratt and I may add that I can find no trace of sexual cells in the siphonozooids. I have confirmed Kismnouye's statement that in C. clatius, the siphonozooids bear the gonads but the specimen of $C$. johnstoni I examined was unfortunately in a condition which renders it impossible for me to state definitely that the autozooids do not bear the gonads.

\section{Corallium Lamarck.}

Madrcpora (pars) Linnaeus, Syst. Nat., X, 1757.

Isis (pars) Linnaeus, System Nat. XII.

Coralliun Lamarck, Mém. Mus. Hist. Nat., I, I \& 5.

Corallimm + Plemrocorallium + Hemicorallium Gray, Catologue of Lithophytes, IS70, p. 22.

Corallim + Plcurocorallim Ridley, Proc. Zool. Soc., I882, p. $22 \mathrm{I}$.

Corallimm + Pleurocorallium + Plcurocoralloides Moroff, Zool. Jahrb. Abth. System., 1902, p.404.

Corallim Kishinouye, Journ. Imp. Fisheries Bureau Japan, XIV, I, I904, p. 20. 
The difficulty of separating the species of Coralliidx into generic groups has been already pointed out by Kisminouve. Neither the occurrence of "opera-glass" shaped spicules, nor the position of the autozooids on one surface of the colony only are satisfactory characters for generic distinction and I agree with the Japanese author that it is better, for the present, to recognise only one genus in the family. Corallizm nobile appears to stand alone as regards the character that the autozooids bear the gonads but it would be premature to suggest that this affords sufficient grounds for separating it from the other species. It would be extremely: interesting to determine whether the specimens of this species taken at the Cape Verde islands are similar as regards the disposition of their gonads to those of the Mediterranean.

The geographical distribution of the species of Corallium may be mentioned here. Corallizm nobile is found in the Mediterranean Sea and off the Cape Verde islands (7). Three species occur off Madeira, C. maderense; C. johnsoni and C. tricolor Johnson (I). C. johnsoni has also been found off the W. coast of Ireland (Hickson, Nature LXXIII, I905, P. 5) and quite recently a specimen of $C$. maderense has been obtained in the Bay of Biscay.

Nine species occur off the coast of Japan (Kishinouye and Moroff). Corallium secundum is said to occur off the Sandwich islands (DANA) but there may be some doubt that this is the correct locality of the specimen. Corallium stylasteroides is found off Mauritius island (RIDLEY). Specimens of dead coral probably belonging to this genus were found by the Challenger at depths of 1525 fathoms in the Atlantic and 310 fathoms off Prince Edward's island.

As I have already pointed out (I905) there is some historical reason for believing that the supply of red coral to the japanese market came from the South seas but there is no evidence as to the exact locality of this fishery.

According to Rumphius the coral was well-known to the Malays of his time by the name Maradjian but it was not found either in the Indian or Red sea. I cannot find any reference to the Coral in Rumphius' Amboynsche Rariteitskamer and I quote the statement given from the German edition by C. F. Wilkens ( 1787 ) of PALLAs' Elenchus Zoophytorum, Vol. I, p. 29 I ${ }^{1}$ ).

The Challenger expedition found dead fragments of a Corallium attributed to C. secundum off Banda 3 Io fathoms and Ki islands 140 fathoms but, so far as I can ascertain, the Sibogaexpedition may claim to be the first to discover living Coralliidæ in the Malay archipelago and therefore the description of the two new species recorded in this memoir is, from the point of view of geographical distribution, of some general interest.

I think it is very probable that Corallizm occurs in the Indian ocean. Alcock states (A naturalist in Indian seas I902, p. 29I) that "M'ARdLE found abundance of a beautiful pink coral, which appears to be identical with the precious coral of the Mediterranean, not far from Trincomalee" "2) and HERDMan found a specimen off the coast of Ceylon, which was unfortunately lost, that may be a Corallium, but no Coralliidxe occurred in the extensive collections of Mr. Stanley Gardiner from the Maldive and Laccadive archipelagoes and I have not found a scientific description of any specimens from other localities in the Indian ocean or the Red sea.

I) Dr. J. Versuul's Jr. has kindly discovered for me the following reference in Rumruus, Herbarii Amboinensis, Liber I2, Cap. 2 "genuinum rubrum Corallium in India orientali non crescit, sed nigri et albi quacdam Species, quas sub prima comprehendimus classe".

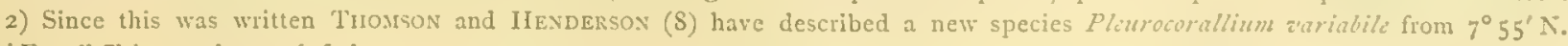
and $8 \mathrm{I}^{\circ} 47^{\prime} \mathrm{E}$, off Trincomalee 506 fathoms. 
1. Corallizm regince Hickson. (Figs I, 2, 3, 4, 8, 10).

S. J. Hickson. Konink. Akad. Wet. Amsterdam, Oct. 12, 1905.

Stat. $280.8^{\circ} 17^{\prime} \cdot 4$ S., $127^{\circ} 30^{\prime} .7$ E. 122 Metres. Fragments.

Stat. ? Label lost. Fragments.

Colony branching irregularly but principally in one plane. Autozooids situated on one surface only except at the extremities of the branches. Autozooids in retraction form irregularly scattered hemispherical verrucie $1,5 \mathrm{~mm}$. in diameter. Siphonozooids very small, in some cases indicated by minute papillx or tubular pores but usually inconspicuous, numerous, unevenly distributed. Gonads borne by the siphonozooids only. Spicules octoradiate, $075-, 08 \mathrm{~mm}$. in length. Colour pink.

The specimens of this species consist of one colony (Fig. I) from Station 280 , considerably broken, which may have been about $80 \mathrm{~mm}$. in height. The base of attachment is $6 \mathrm{~mm}$. in diameter and the main branches are at their bases $4-5 \mathrm{~mm}$. in diameter.

Another specimen (Figs 2 and 3) $37 \mathrm{~mm}$. in height with a diameter at the base of $3 \mathrm{~mm}$, together with two broken off branches, one of which is figured $\times_{3}$ (Fig. 4) were found in another bottle of the Siboga collection the label of which was unfortunately lost or destroyed when the fire broke out in my laboratory.

The axis is very hard and takes a polish as well as that of Corallizm nobile. In transverse section it shows a pale central spot and one or two white rings arranged concentrically round the centre. The colour of the axis is a rather dark pink but has been described as "good" by an expert who examined it. In both specimens there are places where the axis is fistulose, a well-known deformity of growth which in the Japanese corals is due to the action of a commensal Polynoe. I did not discover the worm that causes the deformity in these specimens.

The verruce that mark the position of the autozooids are found principally on one side of the colony as they are in Corallium japonicum, C. sulcatum, C. clatius, C. johnstoni and several other species. At the extremities of the branches a few verrucx may be found on the reverse side but on all the larger branches this side is quite smooth and shows no verrucx.

The verrucx are nearly all hemispherical in form projecting about $1,5 \mathrm{~mm}$. from the surface of the coenenchym and being about $1,4 \mathrm{~mm}$. in diameter. They are scored externally by eight radiating grooves (fig. 4).

The siphonozooids are very small. This is a statement that cannot very well be reduced to figures by the study of preserved specimens. Some of the siphonozooids have an open mouth placed at the extremity of a minute papilla but the coenenchym is so contracted in most places that it is difficult to determine the position of the siphonozooids, the mouth being closed and the verruca withdrawn. Any statement, therefore, as to the size of the mouth might be misleading.

In all the siphonozooids in my series of sections the body cavity is distended with the gonads Fig. IO. There appear to be usually four gonads in each siphonozooid. The colony is male. No ova occur in any siphonozooid examined. One gonad was nearly ripe and this measured , $05 \mathrm{~mm}$. in diameter. The stomodaeum and mesenterial filaments are so distorted by the enlarged gonads that they were difficult to investigate but these structures when compared with those of Corallinm nobilc, have the appearance of being degenerate. I have found that the longest 
diameter of the siphonozooid of $C$. nobile is about $0,15 \mathrm{~mm}$. Allowing for distortion it may be reckoned that the corresponding diameter of $C$. regince is not more than $0,05 \mathrm{~mm}$. This measurement conveys some idea of the relative size of the siphonozooids of the two species but it is one that can only he regarded as an approximate guess.

The spicules, as in all the other species, are very variable in form but the largest and what we may suppose to be the full grown spicules are nearly all of the octoradiate type and about $0,08 \mathrm{~mm}$. in length. If a number of these typical octoradiate spicules are examined critically it is soon discovered that they exhibit great variation in detail. It may take a long time to find a single specimen so regular in form as that shown in figure 7 , in which the extremity $e$ is similar to the extremity $f$ and the rays $a, b, c, d, g, h$ approximately equal in length and diameter, but in the most regular spicules found there are differences in the size and arrangement of the tubercles on the rays and on the extremities. Apart from the varieties of size and arrangement of the tubercles however a great many spicules are found in which the two extremities $e$ and $f$ of the spicule are unsymmetrical, and then if one extremity $e$, is much larger than the other $f$ the spicule becomes club shaped. There is also a frequent variation in the arrangement of the rays. Thus the rays $g$ and $h$ are often situated nearer to or further away from the extremities than the rays $a, b, c, d$. This variation leads to the formation of irregular spicules such as Fig. $8 a$ which have a general family resemblance to the octoradiate spicule but are not typical in development. Another variation is seen in the imperfect development of the rays of one side. (Fig. 8b) and these spicules are very similar to the spicules described by RidLey as "opera-glass" shaped spicules. These differences in the character of the spicules are doubtless due to the variable character of the pressure of the surrounding mesogloea, and the fact that in Corallizm the spicules are so closely crowded together leads to the formation of many remarkable distortions of their growth.

In Corallizm regince the spicules of the variety shown in Fig. Sb, i. e. the opera-glass shaped spicule are comparatively rare. The great majority are somewhat irregular octoradiates similar to Fig. $8 a$ but a considerable number approximating to the typical octoradiate spicule (fig. 7) may be found.

It is quite impossible to give very definitely any statements as to the difference in size between the spicules of this new species and that of any other species of the genus.

The statements made by different observers are not consistent even in the case of such a well known coral as Corallizm nobile of the Mediterranean. DE LACAzE-Dutmiers described the spicules as being $0,05-0,07 \mathrm{~mm}$. in length. Kölitiker (2) states that they may be up to ,09 $\mathrm{mm}$. in length but several spicules in my own preparations of this species are certainly over $0,1 \mathrm{~mm}$. in length. The spicules in my preparations of $C$. regine do not exceed $0,085 \mathrm{~mm}$. and are therefore smaller than those in my own preparations of $C$. nobile, but $I$ feel that it is quite probable that if I made another preparation at random from each of these two species a different result might be obtained.

The only point that is really of any importance is that the opera-glass shaped spicules are rare in C. regine and in that respect the species differs from C. johnstoni, C. inntile, C. pusillum and others and resembles more closely C. japonicum, C. nobile and others. 
The spicules of the tentacles and other parts of the anthocodix in C. reginc are spindle shaped and provided with shallow tubercles Fig. $S c$ and $d$. As none of the autozooids were expanded it is impossible to give any definite statement as to the arrangement of these spindle shaped spicules in the anthocodix.

\section{Corallizm halmaheirense n. sp. (Figs 5, 6, 9).}

Stat. $150.0^{\circ} 6^{\prime} \mathrm{N} ., 129^{\circ} 7^{\prime} .2 \mathrm{O}$. 1089 Metres. 2 Fragments.

The remarks issued by Prof. WEeber concerning this station are as follows "Trawl torn to pieces; fore beam bent; obviously met with very rough bottom". The two fragments of the species obtained may be either the terminal branches of colonies of considerable size broken off by the trawl as it bumped along over the rough ground or they may represent nearly the whole colonies of a diminutive species peculiar to this region. To this initial difficulty, namely our want of knowledge of the size of the colony, in determining the species, may be added the second, that the small size of the fragments affords very few opportunities of studying the variations in the common characters. It is therefore impossible to declare with confidence that this is a new species. If we had larger specimens the species might exhibit stronger affinities with the japanese species Corallium sulcatum or C. boshucusis than the fragments do, but on the other hand they might confirm the distinction of the species. My own opinion is that it is a more convenient course to give for future reference a distinct geographical name to these specimens from the Djilolo passage at the same time admitting that the evidence that the species is really distinct is not convincing; rather than refer them with some hesitation to a known species.

One specimen, which I will call specimen A is $15 \mathrm{~mm}$. in height and the diameter of the axis is $2,25 \mathrm{~mm}$. It has four lateral branches from $5-8 \mathrm{~mm}$. in length with two small branches on one face which are about $3 \mathrm{~mm}$. in length. These facts suggest that if this is a fragment of a large colony, the branching of such a colony would be mainly in one plane, a condition we might expect to meet in a coral from such a great depth. The other specimen (B) is $16 \mathrm{~mm}$. in height, the main axis is half-moon shaped in section with a greatest diameter of $3,75 \mathrm{~mm}$. The longest branch is $13,75 \mathrm{~mm}$. in length; but there are several other smaller branches arising from one face of the coral. This specimen is a great deal more worn than the other, the coenenchym being absent from a considerable portion of the branches.

Judging from the evidence afforded by these two fragments it seems probable that the ramification of this species is mainly in one plane but the terminal branches exhibit secondary branchlets arising on one face of the coral only.

The axis is very pale pink, almost white, in colour but in specimen A there is an excentric core of a darker pink colour. The coenenchym is of an orange-red colour and much darker than the axis. In both specimens there is a distortion of growth due to the presence of an epizoic polychaete worm. In specimen $A$, the worm remains in situ (Fig. 5).

The verrucx of the autozooids are conical in shape very prominent and marked externally by eight deep longitudinal grooves. These verrucie are from $2-3 \mathrm{~mm}$. in height. It cannot be 
said that the verrucze are all turned in the same direction but nevertheless there is clearly a tendency for them to turn towards one face, which in accordance with the nomenclature suggested by Miss England and myself in our memoir on the Stylasterina, I shall call the anterior surface of the coral. A very characteristic feature of the species may be seen in the fact that many of the terminal branchlets (fig. $5 b, c$ ) terminate in a pair of opposite verrucx so that these branchlets have the shape of a capital $T$.

In the characters described above the specimens have some resemblance to Corallinm sulcatum of Kishinouye and to Pleurocorallium tricolor and Pleurocorallium maderense of Jomson. The first named of these species was obtained off Mera, Boshu, Japan from a depth of over 100 fathoms and the last two from deep water off Madeira.

Reference to Kishinouye's (3) figure 2 Plate IV and to Johnson's (I) figures Plate VII fig. I and on p. 58 shows a decided similarity in growth and form to the specimen I have drawn in (figs 5 and 6) but particularly in the character of the $T$ terminal branches. All of these species are rare. Kishinouve has seen only two specimens of $C$. sulcatum and Joinson mentions only three specimens of $P$. tricolor and one of $P$. maderense.

It is difficult to separate definitely our new species from the three named species by the characters so far described, but in the character of the spicules there are differences which may be of importance.

In the coenenchym of $C$. halmahcirense there are numerous crowded spicules of the octoradiate type (Fig. 9a) measuring, when full grown, ,o6-, $0 ; \mathrm{mm}$. in length. They are very similar in form and shape to the spicules of C. regince but are decidedly smaller. In the verrucæ the spicules are flattened spindles or rods (about 0,09 $\mathrm{mm}$. $X, 015 \mathrm{~mm}$.) with many tubercles (Fig. 9).

In the japanese species and in the species from Madeira there appears to be much more variation in the shape of the spicules of the coenenchym. Kishinouye states that in $C$. sulcatum the spicules are of three kinds "sexradiate, septemradiate and double clubs" and Johrson states that there are three forms of spicules in the cortex of Pleurocorallinm tricolor and $P$. maderense of which the opera-glass shaped spicules are the most numerous. Unfortunately neither of these authors gives any statements in the text as to the size of the spicules but judging from the scale of the figures there is not probably much difference in this respect between the spicules of our species and those of the other three. In the two preparations of spicules I have made there is not much variation in the shape of those of the coenenchym. There is not a single one that could be called "opera-glass shaped" and there are none of the irregular form figured by Kishinouye.

It is quite certain that this species is dimorphic. Between the verrucre of the autozooids a number of minute white specks may be seen that are certainly siphonozooids, but as there is so little material at my disposal I have not made any sections to determine the general characters they exhibit or the sexual condition of the colony.

In conclusion I may remark that I think these specimens from the Djilolo passage represent a distinct species but are too fragmentary in character to enable me to give a diagnosis. 


\section{Literature referred to in the text.}

I. Johnson, J. Y. Notes on the Coralliide of Madeira. Proc. Zool. Soc. I899, p. 57.

2. KöLliKER, A. Icones histiologicx. Leipzig, IS65.

3. Kisminoure, K. Notes on the Natural history of Corals. Journal Imp. Fisheries Bureau, Vol. XIV, $\mathrm{N}^{0} \mathrm{I}, \mathrm{IgO4}$.

Containing an account of the chemistry of the axis by S. FUNABASHI on p. IO.

4. De Lacaze-Duthiers, H. Histoire naturelle du Corail. Paris 1864.

5. Moseley, H. N. Quarterly Journal Micr. Science, October 1882, p. 396.

6. Nicholson, H. A. Note on the structure of the skeleton in the Genera Corallium, Tubipora and Syringopora. Ann. N. H., Jan. I 884, p. 29.

7. Wright, E. P. and Studer, TH. Report of the collections of H. M. S. Challenger, Vol. XXXI, I889.

8. Thomson, J. A. and Henderson, W. D. Alcyonarians collected by the R. I. M. S. "Investigator". Calcutta, I906. 
PLATE 


\section{PLATE I.}

Fiv. I. Corallinm regince. A basal fragment from station 2So, anterior surface. $b$ base of attachment. Natural size.

Fig. 2. Corallium regina. A colony from uncertain locality view of anterior surface. The pale pink swellings on the main stem at s. $\overline{\mathrm{s}}$. form the covers for the tunnel of an epizoite. $b$ base of attachment Natural size.

Fiv.s. 3. Corallinm regince. The same colony, as in Fig. 2, posterior surface view.

Fig. 4. Corallim regina. Another fragment as seen from the anterior surface. $\times 3$. showing the hemispherical verrucae.

Fig. 5. Corallimm halmaheirense. a The lower opening of a tunnel containing an epizoic polychaet worm. $b$ and $c$ the typical $T$ terminal branches. $\times 2$.

Fig. (i. Corallium halmaheirense. A branch of the same colony more highly magnified $(\times 3,5)$ to show the conical verrucx and their deep longitudinal grooves.

Fis. 7. Diagram of an octoradiate spicule of Corallium. $\times 400$.

Fig. S. Group of 5 spicules of Corallimm regince. a Irregular octoradiate spicule (abundant), $b$ opera-glass shaped spicule (rare), $c$ and $d$ two spindle shaped spicules from the verruca.

Fir. G. Group of spicules of Corallimm halmahcirense, a Octoradiate spicule. The others spindle shaped. spicules of the verrucx.

I.is. IO. Diagram of a transverse section through a branch of Corallizm reginae. A Autozooid, $S$ Siphonozooid bearing the gonads, $c$ canals in the coenenchym coe, Ax axis. 

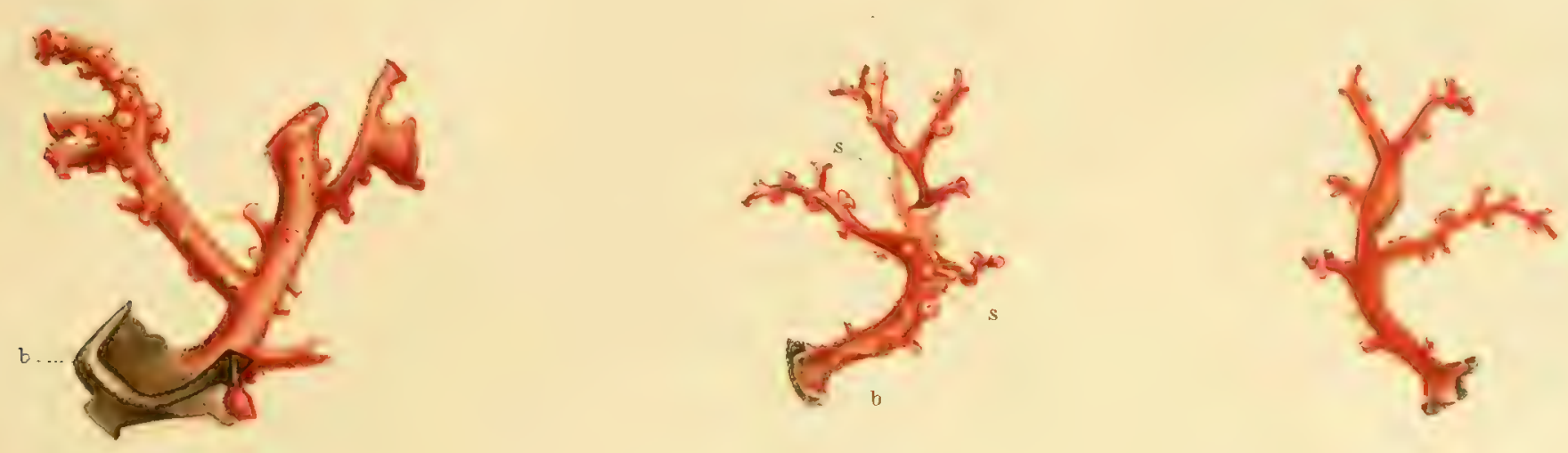

4.
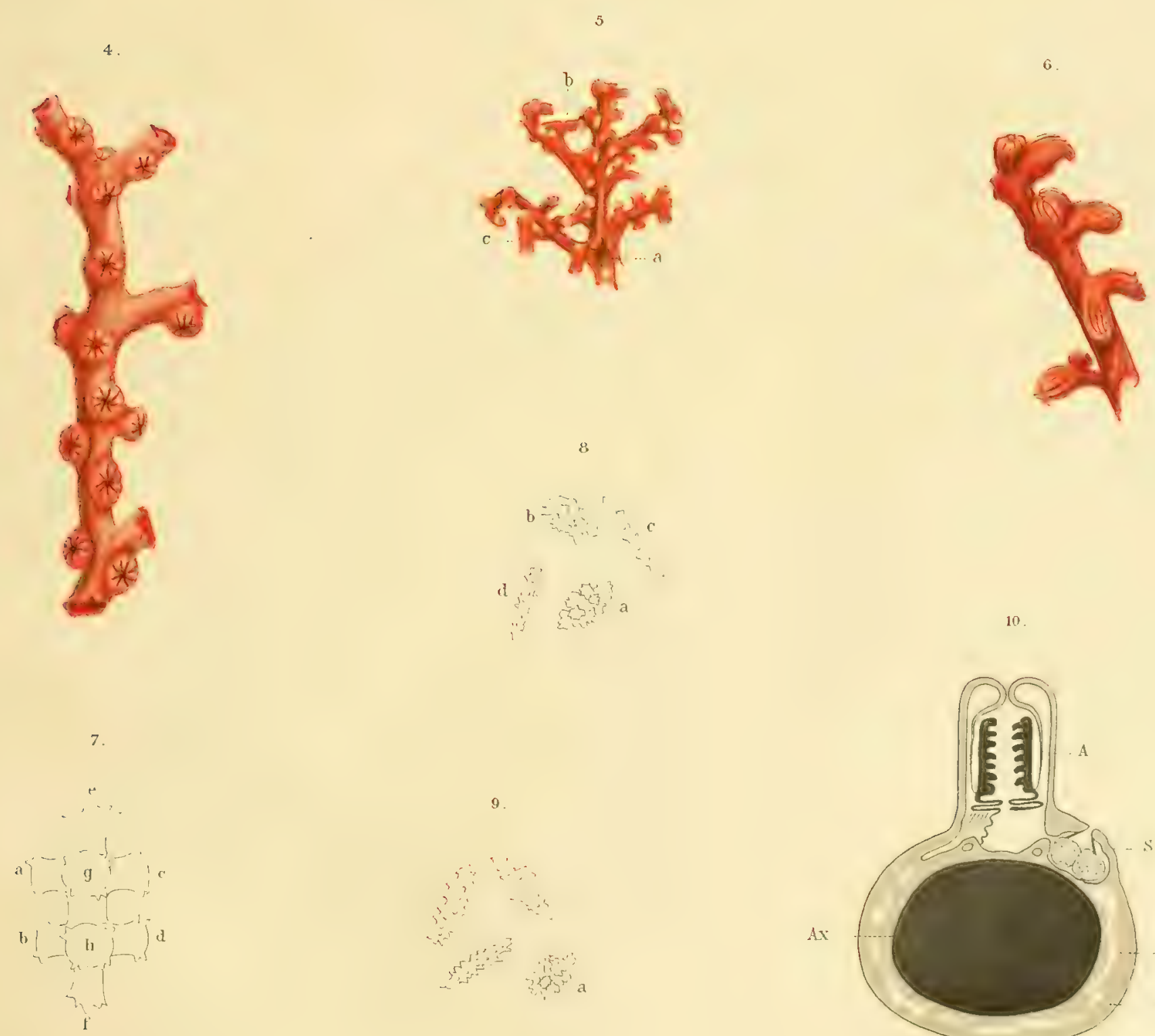

6 



\title{
II. PSEUDOCLADOCHONUS HICKSONI nov. gen. nov. spec.
}

\author{
EIN NEUER TELESTIDE DER SIBOGA-EXPEDITION
}

VON

\section{Dr. J. VERSLUYS,}

Privat-Docent an der Cniversität Amsterdam.

Mit 2 Tafeln und I6 Figuren im Text.

\section{Vorwort.}

Die vorliegende Arbeit enthält die Beschreibung einer Art der Siboga-Sammlung, Pseudocladochonus hicksoni n. gen. n. sp., welche ich zu den Telestidae bringe. Dieselbe zeigt einen so interessanten Bau, so manche Eigentümlichkeiten, dass ich mit der Veröffentlichung einer Beschreibung derselben nicht länger habe warten wollen. So zeigt diese neue Art in den Mesenterien der Polypen die Bildung fester Skeletsepten, wie das bisher noch von keinem Alcyonarier bekannt war. Sehr interessant ist die Umbildung der gestreckten ventralen Abschnitte der Polypen zu Stämmchen, weil dabei die acht Mesenterien in der Achse mit einander verschmelzen und es zu einer Sonderung der Magenhöhle in acht parallele, kreisförmig angeordnete Stammkanäle gekommen ist. Einen solchen Zustand nimmt TH. STuder als Zwischenform an bei seiner Ableitung der Gorgoniden von Telestiden.

Sehr auffallend ist auch die Ähnlichkeit der Siboga-Art mit den paläozoischen Auloporidac, besonders mit Cladochomus M'Coy. Diese Ähnlichkeit ist eine so weitgehende, dass eine mehr eingehende Besprechung derselben mir geboten erschien zur Begründung des Ergebnisses meiner Untersuchung, nämlich: dass diese Ähnlichkeit doch nur auf Convergenz beruht und dass keine engere Verwandtschaft besteht.

Mit Freuden gedenke ich der liebenswürdigen Unterstützung, die mir bei der Herstellung dieser Arbeit von mancher Seite zu Teil geworden ist. Namentlich den Herrn Dr. E. Dusors in Haarlem, Dr. G. J. Hinde, South Croydon, Londen, Sydner J. Hicksos in Manchester, Dr. F. L. Kitciin in Londen, Max IVeber in Amsterdam und C. Ph. Sicuter in Amsterdam bin ich deswegen zu grossem Danke verplichtet.

Das Rheinische Mineralien-Contor, Dr. F. KRaxtz in Bonn, fertigte nach meinen Angaben sehr gute Dünnschliffe durch Material von Cladochonus und Aulopora an. 
Pseudocladochonus hicksoni nov. genus, nov. species.

Stat. I44. Nordküste der Insel Salomakiëe (Damar), bei der Südspitze von Halmaheira. 45 M. Korallenboden und Lithothamnion. Einige Fragmente und eine sehr kleine Kolonie mit Basis. Stat. 166. $2^{\circ} 2 S^{\prime} \cdot 5$ S., I $3 I^{\circ} 3^{\prime} \cdot 3$ O. Ceram-See. I 8 M. Harter, grober Sand. Einige Fragmente.

Von dieser Art liegen mehrere Bruchstücke vor, darunter auch eine kleine Kolonie mit Basis. Ein Fragment mit 3 Polypen wurde in einer Längsschnittserie zerlegt, drei andere Fragmente mit 4, resp. 2 und I Polypen, in Querschnittserien.

Das Material beider Stationen finde ich völlig identisch und ich habe dasselbe denn auch bei meiner Beschreibung nicht aus einander gehalten.

\section{ERSTER TEIL.}

\section{Beschreibung der Art.}

\section{Gröberer Bau.}

Die Art ist zart (Textfig. I), von weisser Farbe (in Alcohol) und bildet aufrecht wachsende,

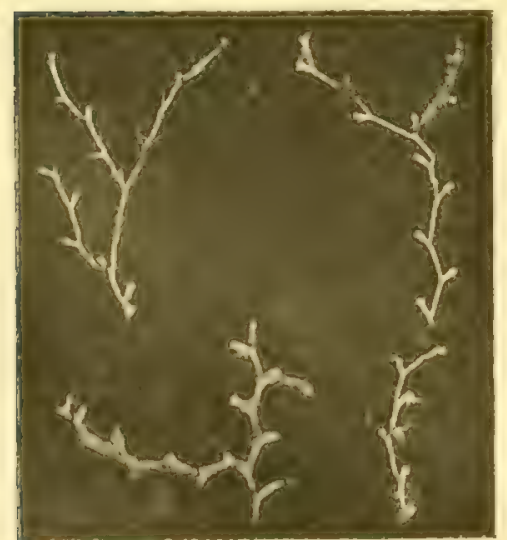

Fig. 1. P'sulucladochonus hicksoni. Fragmente in wahrel Grösse; A, I) und 1$)$ voln Stat. 144, D) mit Basis; C von Stat. I 66 roa einer Spongie iberwachsen. annäherend in einer Ebene ausgebreitete, nicht dicht verzweigte Kolonien. Die Stämmchen und Äste sind mehr oder weniger B deutlich zikzak-förmig gebogen und tragen an den Ecken die Polypen. Letztere liegen meist in der Verzweigungsebene der Kolonie, abwechselnd in 2 Längsreihen (Textfig. I; Taf. 3, Fig. Io). Die einzige vorhandene Basis einer Kolonie zeigt das cylindrische Stämmchen mit etwas lappiger Basalausbreitung einer kleinen Muschelschale aufgewachsen (Taf. 3, Fig. 14). Die Festigkeit, welche erfordert wird um sich über den Boden erheben zu können, verdankt die Art der Anwesenheit eines oberflächlichen Skeletcylinders, welcher sowohl die Stämmchen wie die Polypen umgiebt; dazu gesellt sich namentlich in den Stämmchen noch ein inneres Skelet. Dieses Skelet wird von fest verschmolzenen Kalkkörperchen gebildet.

Die Stämmchen zeigen etwas oberhaḷ der Polypen fast immer ein gelbliches Querband; 
letztere sind in Fig. Io auf. Taf. 3 durch Punktirung angegeben. Nicht selten zeigen die Stämmchen hier eine Einschnuirung. Während der Skeletcylinder sonst überall sehr fest ist, bricht er an diesen Stellen sehr leicht ab. Dabei fällt die Kolonie in Segmente aus einander, wie das auf Taf. 3 in Fig. Io bei G abgebildete; jedes Segment besteht aus einem kleinen Abschnitte des Stämmchens dem seitwärts, unweit des oberen Endes, ein schräg gestellter Polypenkelch aufsitzt. Auch sind die Stämmchen an diesen gelblichen Querbändern etwas biegsam, wodurch eine überaus grosse Starrheit der Kolonien, wie sie sonst durch ein festes Skelet bedingt sein würde, vermieden ist. Bisweilen liegt eine solche Biegungsstelle auch an der Basis eines Polypen (Taf. 3, Fig. IO, B, rechts unten). An diesen Stellen unterbleibt die feste Verschmelzung der Kalkkörperchen des Skeletes; die gelbliche Farbe deutet vielleicht auf die Anwesenheit einer grösseren Menge organischer Substanz (Hornsubstanz?) hin, als sonst im Skelete zu finden ist.

Die mikroskopische Untersuchung zeigt, dass das Innere der hohlen Stämmchen durch Scheidewände in Längskanäle verteilt ist (Tåf. 3, Fig. I 3). Diese Scheidewände enthalten auch ein Skelet von verschmolzenen Kalkkörperchen; nur an den gelblichen Querringen, den Biegungsstellen, finde ich diese Kalkkörperchen nicht verschmolzen. Bei Betrachtung mit der Lupe oder bei schwacher Vergrösserung unter dem Mikroskope bei auffallendem Lichte sieht man durch die etwas durchscheinende Oberfläche der Stämmchen hindurch diese Scheidewände als milchweisse Längsbänder. Es finden sich meist 8 annäherend gleich weite Längskanäle, oder 4 weitere wechseln ab mit 4 engeren (Taf. 3, Fig. I 3), oder die Kanäle sind mehr unregelmässig èntwickelt und verschmelzen teilweise, wodurch deren weniger als 8 vorhanden sind. Am unregelmässigsten finde ich die Kanäle oberhalb der Polypen; unterhalb eines Polypen dagegen zeigen meine Querschnittserien immer 8 Längskanäle, obwohl oft von etwas verschiedenem Durchmesser (Taf. 3, Fig. 9).

Dieses Kanalsystem setzt sich nicht continuirlich durch die Stämmchen fort, sondern es ist bei jedem Polypen unterbrochen, indem die Polypenbasis sich zwischen die Kanäle des nächst unteren und oberen Stammabschnittes einschiebt (Taf. 3, Fig. I I). Das Kanalsystem eines zwischen zwei Polypen liegenden Stammabschnittes geht unten aus der Seite des

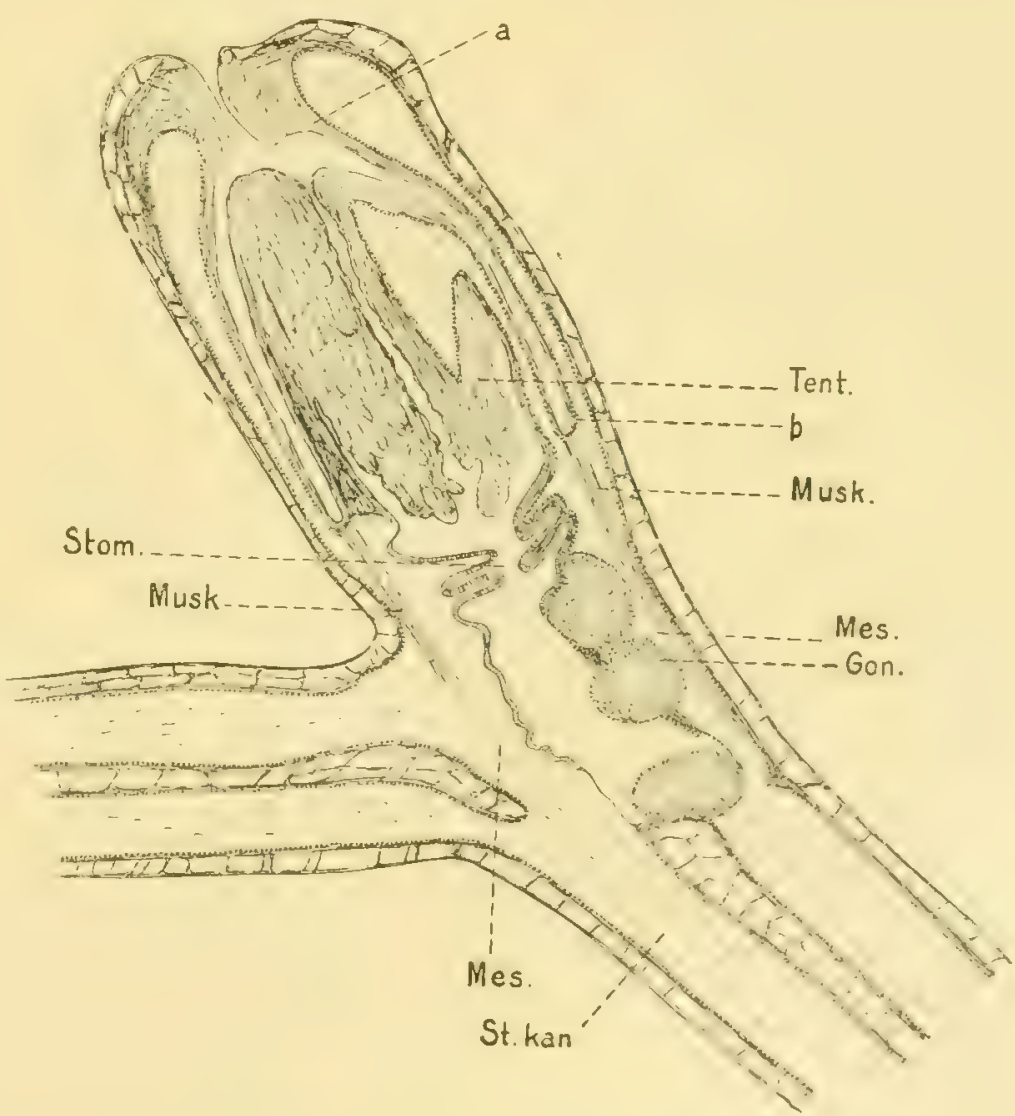

Fig. 2. Psculocludochomus. Läugsschnitt durch einen entkalkten l'olypen, nach einigen Schnitten combinirt; etwas schematisch.

Tent. Tentakel; Musk. Muskel; . Mis. Mesenterium; Gon. Gonade; St.kan. Stammkanal; Stom. Stomodacum; a bis " nach innen umgeschlagene Seitenwandung des l'olypen. \ 57. einen Polypen hervor und setzt sich oben unmittelbar in die Magenhöhle des nächst oberen 
Polypen fort. Letzterer Polyp ist in der directen Verlängerung des Stammabschnittes gelegen und die S Stammkanäle münden genau zwischen den \& Septen (Mesenterien) des Polypen (vergl.

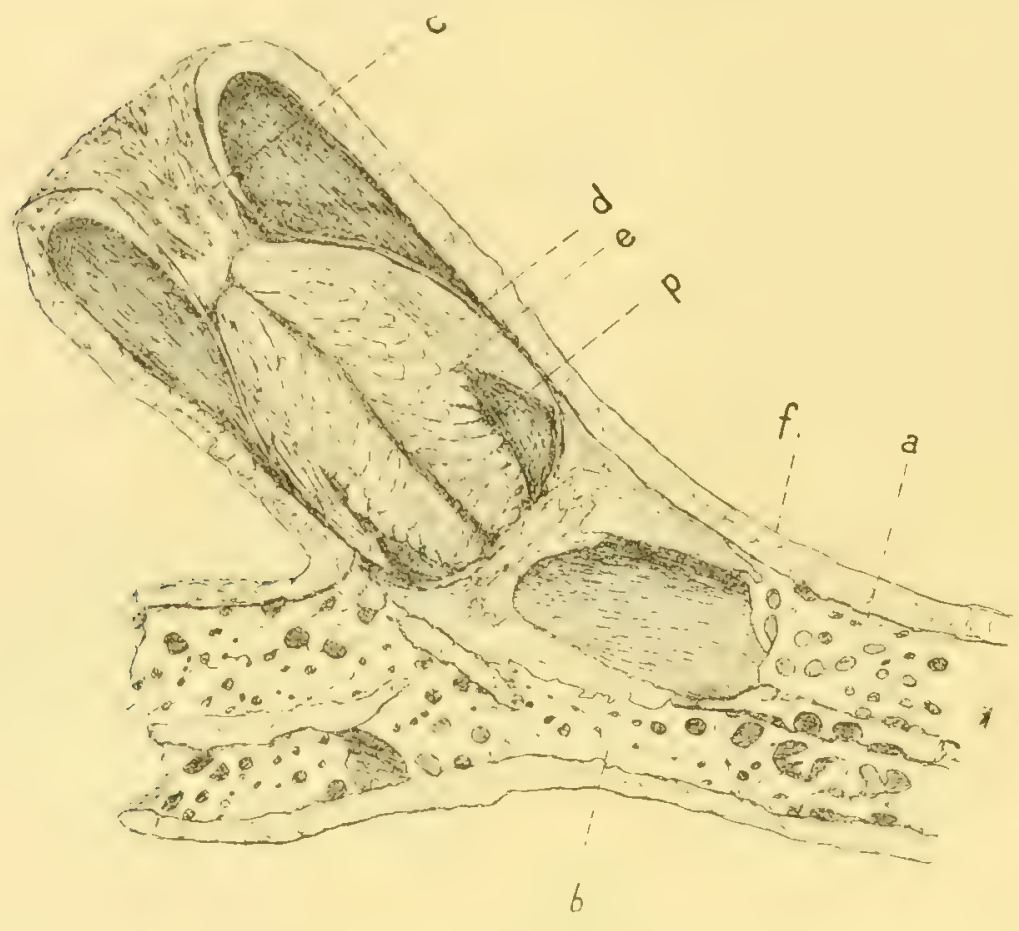

Fig. 3. Pseudocladochonus. Lüngsschliff durch einen contrahirten Polypen. a Skelet in der Scheidewand zwischen 2 Stammkanälen; b Skelet in einem der dorsalen Mesenterien des Polypen; $c$ nach innen ungeschlagene Seitenwandung des Polypen; $d$ Tentakelbasis; $c$ distaler Abschnitt eines Tentakels $f$ Übergang des Polypenkelches im Stammabschnitte; $p$ pinnulae der Tentakel. $\times 57$

letzterem hervorgehenden Stämmchen. Bei meinem Materiale finde ich keinen Beispiel dafür, dass ein Polyp mehr als 2 Stämmchen aus sich hervorgehen lässt.

Sowohl die Grösse der Polypen als auch die Länge der dieselben verbindenden Stammabschnitte wechseln erheblich; Fig. ı A, Taf. 3, giebt die Abbildung eines Fragmentes mit kurzen Stammabschnitten, Fig. IOB diejenige eines Fragmentes mit besonders grossem Abstand zweier benachbarter Polypen. Auch in Textfig. I, S. 2, ist der Unterschied im Abstand der Polypen zwischen den in A und B einerseits, in C andererseits abgebildeten Fragmente deutlich. Beim Materiale der Station 144 variirt die Länge der zwischen je zwei Polypen liegenden Stammabschnitte von 2 bis $5 \mathrm{~mm}$, ist meist etwa $3 \mathrm{~mm}$; beim Materiale der Station $\mathrm{I} 66$ wechselt diese Länge zwischen $\mathrm{I}^{1} / 2$ und $3 \mathrm{~mm}$., mit einem Mittel von nur etwa $1^{3} / t_{t}-2 \mathrm{~mm}$..

Die Polypen bestehen aus einem basalen, nicht zurückziehbaren Kelchabschnitte, in dessen Wandung wir dasselbe feste Kalkskelet finden, wie in den Stammabschnitten, und einem in jenem Kelche bei meinem Materiale zurückgezogenen distalen Abschnitte (Textfig. 2 und 3). Die Länge, bis zu welcher sich der ganze Polyp ausdehnen kann, lässt sich natiirlich nicht bestimmen. Die Länge des festen, basalen Kelches wechselt etwas, ist aber ungefähr $2 \mathrm{~mm}$. beim Nateriale beider Stationen 144 und 166. Meist ist der Querdurchmesser der Polypen etwa I mm., und etwas grösser als derjenige der Stammabschnitte (Fig. Io A, Taf. 3), doch kann derselbe gleich gross sein (Taf. 3, Fig. 10 C). die Textfiguren 2 und 3 , und Taf. 3 , Fig. I I, sowie die Figuren 9 und 8 , Eine Verzweigung des Stämmchens entsteht, wenn aus einem Polypen an 2 Stellen ein Stammabschnitt hervorwächst. Die beiden Abgangsstellen derhoch gelegen und die Stämmchen gehen nach entgegengesetzten beiten ab; das untere ist die eigentliche Fortsetzung des Stämmchens, das obere, welches dem Rande des Polypenkelches ziemlich genähert sein kann, bildet den Seitenzweig (Taf. 3, Fig. 10 A, bei Z). Der Rumpf jener Polypen, welche 2 Stämmchen abgeben, ist länger als gewöhnlich. Die Biegungsstelle in Figur Io B, Taf. 3 (rechts unten, punktirt) liegt nicht im Stämmchen, sondern in einem Polypen zwischen den beiden aus selben finde ich immer verschieden 
Die meisten Fragmente der Station 166 sind von einer in dünner Schicht uber sie ausgebreiteten Spongie bedeckt, wodurch diese Fragmente bei oberflächlicher Betrachtung einen kräftigeren Bau zu zeigen scheinen, als tatsächlich der Fall ist (Textfig. I, C, S. 2).

\section{S. Nähere Angaben über den inneren Bau.}

Die Polypen sind typische Alcyonarier-Polypen mit 8 gefiederten Tentakeln, einem Stomodaeum mit einer ventralen Siphonoglyphe (Fig. I und 2, Taf. 2), mit 8 Mesenterien, auf deren ventralen Flächen die Längsmuskeln hervorragen, mit ectodermalen Gastralfilamenten an den dorsalen Mesenterien (Fig. 3-6, Taf. 2). In den dorsalen Mesenterien werden keine Gonaden gebildet.

Die Tentakelkrone ist als ganzes im basalen Polypenabschnitte zuruickgezogen (Textfig. 2 und 3 , S. 3 und 4 ) wie das auch bei anderen Alcyonariern der Fall ist, z.B. bei Telesto prolifera ${ }^{1}$ ).

Basalwärts werden die Mesenterien, namentlich die ventralen, schmäler und verlieren ihre Gastralfilamente. Ein ganz wenig weiter nach unten aber nimmt plötzlich die Breite der Mesenterien wieder zu und statt mit freiem Rande in die Magenhöhle der Polypen vor zu ragen, treten die Mesenterien mit einander in Verbindung (Fig. 7, Taf. 2, Fig. 8, 9, Taf. 3), sodass die Magenhöhle des Polypen in die 8 geräumigen Kanäle des Stämmchens übergeht. Die trennenden Scheidewände dieser letzteren sind die unmittelbare Fortsetzung der 8 Mesenterien des Polypen (Textfig. 2, 3; Taf. 3, Fig. I I Sch.). Der Übergang der Polypen in die dieselben tragenden Stämmchen ist ein plötzlicher.

Dass die Weite der 8 Stammkanäle eine sehr verschiedene sein kann, wurde schon oben angegeben, auch das Verschmelzungen auftreten, namentlich im basalen Abschnitte der Stämmchen, sodass die Zahl der Kanäle sich bis auf 6 und sogar 5 verringern kann.

Basal münden die Kanäle jedes Stammabschnittes seitwärts in die Magenhöhle des Polypen, aus dessen Seitenwandung das Stämmchen hervorgewachsen ist. Einige der Stammkanäle münden unten mit 2 Öffnungen, anderen gehen zu zweien aus einem gemeinsamen Anfange hervor. Ich zähle 6 Öffnungen an der Basis des Stämmchens in 4 Fällen, von welchen mir Schnittserien vorliegen, nur einmal 7 Öfnungen. Die Verhältnisse sind aber nicht sehr einfach und mein Material gestattet es mir nicht einen Urteil dariber auszusprechen, ob in der IVeise, in welcher die Stammkanäle seitwärts aus einem Polypen hervorgehen, eine gewisse Gesetzmässigkeit herrscht, oder nicht. Ein durch Kochen in Kalilauge angefertigtes Skeletpräparat vom Abgange eines Stämmchens aus einem Polypenkelche zeigt auch keine genau symmetrische Lage der Öffnungen, mit welchen die Stammkanäle aus dem Polypen hervorgehen; unten liegen drei grössere Öffnungen, durch 2 Skeletsepten (welche in den 2 dorsalen MIesenterien liegen) getrennt; über jedem derselben liegt noch eine etwas kleinere Öfnung (abgebildet Taf. 3, Fig. I I B, bei S), dazu an einer Seite und in der Mitte vielleicht noch je eine sehr kleine, doch ist es möglich, dass letztere beiden Öffnungen beim lebenden Polypen durch Mesogloea verschlossen gewesen sind. Bei allen fünf, nur ein Stämmchen abgebenden P'olypen, von welchen mir Schnittserien

1) vox Kocut. Morphol. Jahrb. Bd. 7, 1SS2, Iaf. 12, Fig. 7. 
vorliegen, geht das Stämmchen von der dorsalen Polypenseite ab, d. i. von derjenigen Seite, welche durch die beiden Mesenterien mit ectodermalen Gastralfilamenten gekennzeichnet ist. Bei cinem anderen Polypen, aus dem zwei Stämmchen hervorgehen, wo also eine Verzweigung der Kolonie stattfindet, geht das obere der beiden Stämmchen, wahrscheinlich also der am spätesten grebildete Seitenast, von der ventralen Polypenseite ab, wie mir eine Schnittserie zeigt. Demnach muss das diametral entgegengesetzte untere Stämmchen zweifellos aus der dorsalen Polypenseite hervorgegangen sein. Dieser Teil der Schnittserie ist leider verloren gegangen, doch lässt der Abgang des oberen Stämmchens hieriber keinen Zweifel. Warscheinlich geht also immer die Fortsetzung des Stammes von der dorsalen Polypenseite ab; entspringt dann etwas höher hinauf noch ein zweites Stämmchen aus demselben Polypen, so geht dieses aus der ventralen Polypenseite hervor.

Es muss nun noch die Frage beantwortet werden, welcher Natur die Stammabschnitte sind, ob dieselben als Stolonen gedeutet werden müssen, oder ob sie umgeänderte, basale Abschnitte von Polypen sind. Dass die Scheidewände die unmittelbare Fortsetzung der Mesenterien der Polypen sind, spricht entschieden dafür, dass diese Scheidewände tatsächlich hervorgregangen sind aus Mesenterien, welche in der Achse der Magenhöhle sehr langer Polypen mit einander verschmolzen sind. Dass die Scheidewände dabei etwas unregelmässig entwickelt sein können, scheint mir kein Grund gegen diese Ableitung zu sein, weil das sehr gut als secundäre Veränderung gedeutet werden kann. Verschmelzung einiger Mesenterien in der Längsachse der MIagenhöhle weisen bekanntlich auch die axialen Polypen der Pennatuliden auf und KöLtiker hat dasselbe von Pseudogorgia, einem eigentümlichen Telestiden beschrieben ${ }^{1}$ ).

Eine Deutung der Stammabschnitte als Stolonen wäre auch zu verteidigen; sie giebt aber keine Erklärung für die Regelmässigkeit des Kanalsystemes in den Stammabschnitten und für den Übergang der Mesenterien in die Scheidewände zwischen den Stammkanälen. Dass die Polypen einer Anzahl geräumiger, einander paralleler Entodermkanäle (Solenia) terminal aufsitzen, kennt man nur von Heliopora und dort fehlt jede Übereinstimmung in der Lage der 8 Mesenterien und der zahlreicheren Scheidewände, welche die Entodermkanäle trennen. Ich glaube, dass diese Gründe genügen bei Pseudocladochon an der Homologie der Stammabschnitte mit basalen Abschnitten von Polypen und der Scheidewände zwischen den Stammkänalen mit Mesenterien fest $z u$ halten. Die unregelmässigen Öffnungen, mittelst denen die Kanäle der Stammabschnitte sich in die Magenhöhlen der nächst unteren Polypen öfnen, sind dann als Stolonenkanäle, als Solenia (Bourne) zu deuten (vergl. Fig. I I, Taf. 3). Diese Deutung des Baues von Pseudocladochoms erlaubt, wie wir weiter unten sehen werden, einen Vergleich dieser neuen Art mit Telesto, mit welchem Genus sie auch sonst Vieles gemeinsam hat.

\section{Das Skelet.}

a. A ufbau des Skeletes. Das Skelet von Psendocladochonzes ist ein typisches AlcyonarierSkelet; es wird gebildet von Kalkkörperchen, welche im Coenenchym, in der Mesogloea, liegen.

I) Verhandl. der phys. med. Ges. Wüirburg, X. IV. IBd 2, IS72, p. II $2 \mathrm{~S}$. 
In den Stämmchen und in der Wandung der Polypen, soweit letztere nicht samt der Tentakelkrone zuruickgezogen werden können, verschmelzen diese Scleriten mit einander und bilden dadurch eine feste, schützende Hülle, wodurch allein es möglich ist, dass die Kolonien beim geringen Durchmesser ihrer Stämmchen sich ganz frei uiber dem Boden erheben.

Die Form der Kalkkörperchen, welche dieses Skelet bilden, ist die eines dicken Stabes mit verbreiterten und meist gegabelten Enden (Textfig. 4 bei $b$, und Textfig. 5 bei $S_{c l}$.). Ihr grösster Durchmesser ist etwa $0,15 \mathrm{~mm}$.. Sie liegen in einer einzigen Schicht, mit den Enden einander beriihrend oder etwas über einander greifend. Die Lücken, welche zwischen den

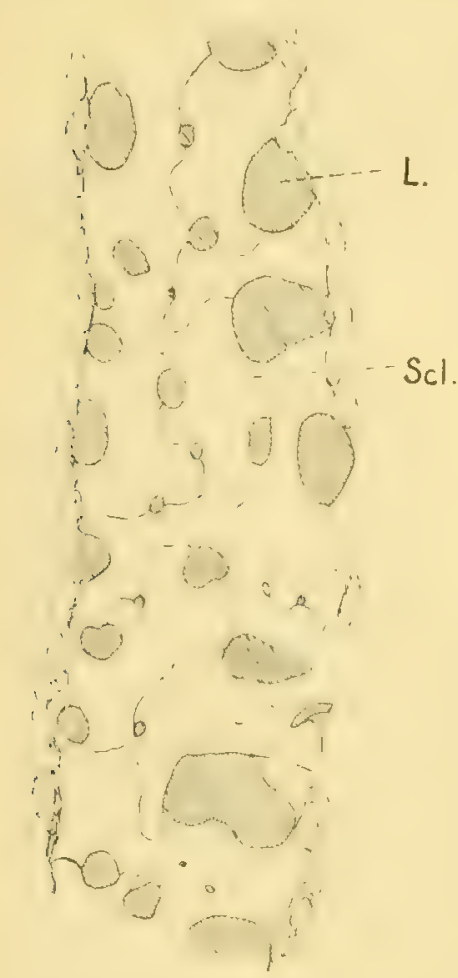

1iig. 6. Pseudocladochomus. Skelet aus einer der Scheidewände zwischen 2 Stammkanälen; das Skelet ist nahezu ausgewachsen. Die Scleriten sind dick, mit einander verwachsen und werden durch meist nur kleine Löcher getrennt; Sct. Scleriten; I. I,och im skelet. $\times 232$.

Die Verlöthung der Scleriten ist zuletzt eine so vollständige, dass auch bei längerem nicht mit anderen Scleriten verwachsen sind; $c$ sus verschmolzenen Scleriten bestehender Abschnitt des Skeletes; $d$ I och, welches zwischen zwei in der Verwachsung begriffenen Scleriten übrig geblieben ist. X 232 .

bleiben, sind in Anfange ziem-

lich gross (Textfig. 4 bei $a$ und $b$; Textfig. 5); da aber die Scleriten allmählig dicker werden, so werden die Lücken zwischen denselben entsprechend kleiner, sodass zuletzt nur rundliche, oft sehr kleine Löcher zwischen den Scleriten ubrig bleiben. Dabei werden auch die Grenzen der Scleriten weniger deutlich, sodass streckenweise sogar eine nahezu continuirliche Skeletschicht entsteht, welche von zahlireichen, meist aber nur kleinen, rundlichen Löchern durchbohrt ist. In Textfigur 4, in welcher ein kleiner Abschnitt des Skeletes vom Rande eines Polypenkelches dargestellt ist, sieht man deutlich, wie in der Nähe des mit 6 bezeich. neten Scleriten die Grenzen der Kalkkörper noch deutlich und die dieselben trennende Liicken noch ziemlich gross und unregelmässig, dagegen bei $c$ die Liicken viel kleiner und die Grenzen der Scleriten undeutlich geworden sind. Dieser Alters. unterschied im Skelet geht auch sehr klar hervor aus der Vergleichung der Textfiguren 5 und 6 .

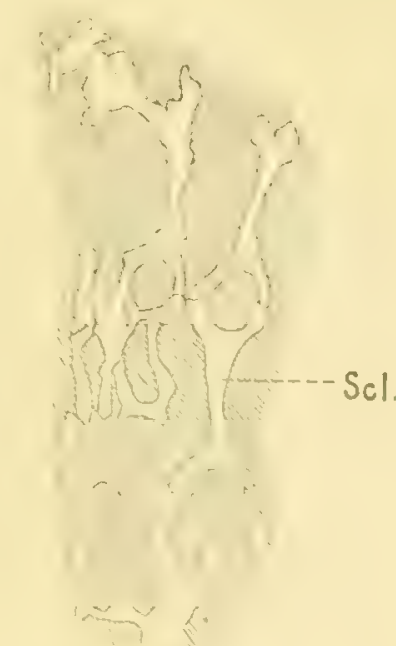

Fiv. 5. Psiudectadictionus. Skelet ats einer der Sicheidcwinde zwischen a Stammkanilen, bei ciner lbictungsstelle. Das Skelet ist erst in infang sciner bildung: die scleriten (Scl.) sind schlank. nicht mit einander verwachsen, und werden durch weite /wischentiumo (gestrichelt) getrennt. $\times 232$.

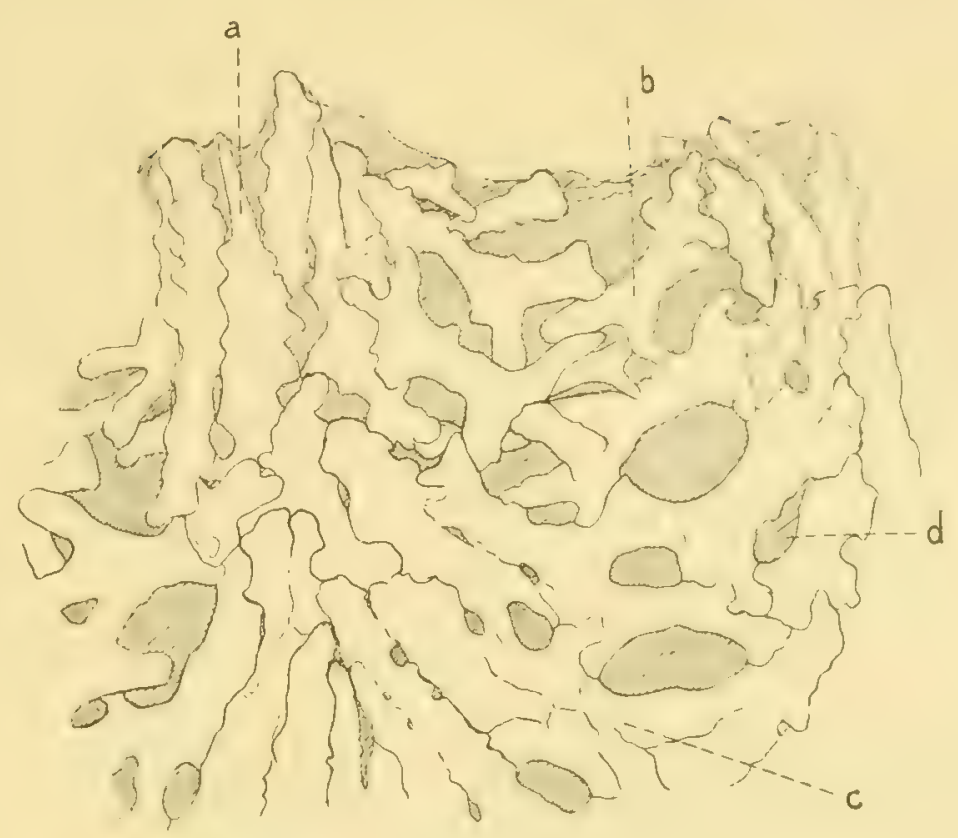

Fig. 4. Pscudocladochonus. Skeletabschnitt vom Rande eines Polypenkelches; $a$ und b Scleriten aus der Nähe des Kelchrandes, welche noch 
Kochen in Kalilauge dieselben sich nicht von einander trennen. Dies weist darauf hin, dass die Scleriten nicht einfach an einander geschmiegt oder durch organische Substanz mit einander verkittet sind. Es müssen die Kalkkörperchen wirklich mit einander verschmolzen sein, indem dort, wo zwei Scleriten sich zuerst nur berühren, später eine beide verbindende Ablagerung von Kalksalzen stattfindet. Setzt das Dickenwachstum sich dann noch weiter fort, so wird es zur Absetzung einer über beide Scleriten ohne Unterbrechung sich ausdehnenden Kalkschicht kommen muissen. Dafür spricht auch die geringe Deutlichkeit der Grenzen der Scleriten in älteren Skeletabschnitten. Bei noch weiter fortgesetztem Dickenwachstum des Skeletes (welche bei Pscudocladochonus nicht stattfindet) würde der spiculäre Bau desselben vollständig verloren gehen; es wïrde anscheinend sogar zur Ablagerung continuirlicher Kalkschichten kommen können. Dies ist wichtig, weil es darauf hinweist, dass die Umbildung eines aus isolirten Scleriten bestehenden Skeletes zu einem aus continuirlichen, concentrischen Schichten aufgebautem Skelete vielleicht doch nicht so unmöglich ist, als es auf den ersten Blick erscheint.

6. Verbreitung des Skeletes. Das feste, aus verschmolzenen Scleriten aufgebaute Skelet umgiebt als schützende und stützende Hülle die Stämmchen und bildet die Polypenkelche. Es bleibt aber dieses Skelet nicht auf der Oberfläche der Kolonien beschränkt, sondern es ist in ganz derselben Form in den Scheidewänden entwickelt, welche in den Stämmchen die weiten Längskanäle von einander trennen. So bildet das Skelet 8, oder bei teilweiser Verschmelzung der Längskanäle, nur 7,6 oder 5 mehr oder weniger regelmässige Skeletplatten, welche in der Mitte der Stämmchen zusammentreffen und sich dort zu einer centralen Skeletsäule vereinigen. In Fig. I 3, Taf. 3 ist dieses Skelet auf dem Querschnitte dargestellt. Die Textfiguren 5 und 6, welche die Anlage des Skeletes in der Form isolirter Scleriten resp. dessen fertige Ausbildung zu einer festen, durchlöcherten Skeletplatte wiedergeben, beziehen sich auf das Skelet jener Scheidewände.

Merkwürdigerweise erstreckt sich dieses Skelet der Scheidewände auch unverändert in die vier dorsalen Mesenterien der Polypen, also in das Mesenterienpaar mit ectodermalen Gastralfilamenten und in das jederseits benachbarte Mesenterium. Ich habe schon oben angegeben, dass die Mesenterien der Polypen die unmittelbare Fortsetzung der Scheidewände der Stammkanäle bilden. Die Seite der Polypen, in welcher diese Sclerosepten liegen, ist immer diejenige, aus welcher die Fortsetzung des Stammes, der nächst obere Stammabschnitt, hervorgeht. Eines dieser Sclerosepten ist in Textfig. 3 bei $b$ (S. 4) abgebildet. Sehr deutlich ist dieses mesenteriale Skelet auch auf clen Querschnitten erkennbar, da man die Lakunen, welche das Skelet beim Entkalken zurück gelassen hat, nicht nur in der äusseren Wandung der Polypen, sondern auch in den 4 dorsalen Mesenterien findet (vergl. Taf. 2, Fig. 5, 6 und 7, Mes. dors. und Mes.; Taf. 3, Fig. 8). Es ist dies, so weit mir bekannt, der erste Fall, dass bei einem Alcyonarierpolypen ein inneres, Sclerosepten bildendes Skelet beobachtet ist. Durch ihren Aufbau aus anfänglich isolirten, mesodermalen Scleriten und durch die Lage in den Mesenterien sind diese Sclerosepten von den typischen Sclerosepten der Hexacoralliae grundverschieden.

Durch das innere Skelet wird sowohl die Festigkeit der Stämmchen wie der Zusammenhang der auf einander folgenden Stammabschnitte beträchtlich erhöht. Dass nur an jener Seite der Polypen, aus welcher die Fortsetzung des Stämmchens hervorgeht, das innere Skelet auch 
in den Mesenterien der eigentlichen Polypen ausgebildet ist, steht damit in Einklang, dass an die Festigkeit dieser Polypenseite auch die grössten mechanischen Ansprüche gestellt werden. Durch die Skeletbildung in diesen Mesenterien wird das innere Skelet der einander folgenden Abschnitte eines Stammes mit einander in Zusammenhang gebracht. Die Verhältnisse des inneren Skeletes bei einem Polypen, aus dem zwei Stämmchen hervorgehen, habe ich wegen Mangels an Material nicht untersucht.

c. Die Biegungsstellen im Skelete. Die Verschmelzung der Scleriten zu einem festen Skelete unterbleibt, wie schon oben angegeben wurde, an besonderen ringförmigen Stellen des Stammskeletes (die punktirten Querbänder in Fig. IO, Taf. 3). Im Skelete der Polypenkelche sind diese Biegungsstellen selten. Ich finde das wenigstens bei meinem Materiale nur bei zwei Polypen, aus welchen je zwei Stänmchen hervorgehen; es liegt die Biegungsstelle bei diesen Polypen zwischen den Abgangsstellen der zwei Stämmchen (Taf. 3, Fig. Io B, unten). Die Scleriten erreichen an den Biegungsstellen nicht die übliche Dicke und verschmelzen nicht mit einander; das Skelet steht an diesen Stellen auf junger Bildungsstufe (vergl. Textfig. 5) und es ist möglich, dass hier Längenwachstum der Stammabschnitte stattfindet. Leider kann ich die Frage, wo das Längenwachstum bei Psendocladochonus stattfindet, nicht sicher beantworten. Es ist klar, dass die bald auftretende Verlöthung der Scleriten zu einem festen Skelete bei allen nicht sehr jungen Abschnitten der Kolonien ein Wachstum durch Streckung der Gewebe ausschliesst. Aber sowohl ein terminales Wachstum der Stammabschnitte als auch ein intercalares, das an den oben erwähnten Biegungsstellen statthat, scheint a priori möglich. Die schon hervorgehobene junge Bildungsstufe des Skeletes an den Biegungsstellen steht mit einem intercalaren Wachstum in Einklang, reicht aber ohne weiteres nicht aus, zu beweisen, dass dasselbe auch stattfindet. Die Biegungsstellen sind noch deutlich bei gut ausgewachsenen, basalen Abschnitten der Stämmchen, welche ihr Längenwachstum warscheinlich schon eingestellt haben. Doch könnte die Erhaltung dieser Stellen auch bei vollendetem Wachstum in Zusammenhang stehen mit der Bedeutung derselben als Biegungspunkte, wodurch eine allzugrosse Starrheit der Kolonien vermieden wird. Ich sehe in ihrer Persistenz also noch keinen Grund, den Biegungsstellen eine Bedeutung für das Längenwachstum der Stämmchen als unwarscheinlich abzusprechen. Die sehr wechselnde Länge der zwischen je zwei Polypen liegenden Stammabschnitte macht es unmöglich, aus der Länge derselben auf ein intercalares Wachstum, resp. ein Fehlen desselben, zu schliessen. Ich finde aber dennoch einen Punkt, der für ein intercalares Wachstum der Stammabschnitte spricht, welches dann aber nur an den Biegungsstellen stattfinden kann. Es ist nämlich warscheinlich, dass jeder Stammabschnitt vom Anfang an in einem Polypen endet; denn der Stammabschnit ist ja nur ein basaler Abschnitt eines Polypen (vergl. S. 6). Da nun aber der Bau des Stammabschnittes ein anderer ist, kann durch terminales Längenwachstum des Skeletes am oberen Rande der Polypenkelche nur der Polypenkelch, niemals aber der Stammabschnitt an Länge zunehmen. Da ich bei meinem Materiale immer auch am Ende der nicht abgebrochenen Stammabschnitte einen Polypen finde, so ist ein intercalares Längenwachstum der Stammabschnitte warscheinlich. Für eine Entscheidung reicht aber mein Material nicht aus und will ich auf diese Frage deshalb hier nicht weiter eingehen.

d. Das Skelet in den contractilen Abschnitten der Polypen. Der distale 
Abschnitt der Polypen kamn in den proximalen Abschnitt, in den basalen Kelch, zurückgezogen werden (vergl. Textfig. 2 und 3; S. 3 und 4). Diesen contractilen Abschnitten der Polypen fehlt selbstverständlich ein festes Skelet. Wenn die Tentakelkrone in den Polypenkelch zurïckgezogen wird, wird auch der contractile Abschnitt der Seitenwandung (des Mauerblattes) der Polypen nach innen umgeschlagen (Textfig. 2, S. 3, $a$ bis $b$ ). In diesem Wandabschnitte liegen nur oben noch Scleriten ('Textfig. 3 , bei $c$ ); es sind kleine, circa 0,09 bis O, I $3 \mathrm{~mm}$. lange, spärlich bedornte Nadeln, welche mehr oder weniger deutlich eine Anordnung in \& Längsbändern zeigen. Der basale Teil der Rückenwandung der Tentakel wird von 0,18 bis $0,22 \mathrm{~mm}$. langen, abgeplatteten Nadeln bedeckt (Textfig. 3, bei $d$ ); dieselben sind schwach bedornt, und zwar die am meisten basalwärts liegenden Scleriten noch weniger als die andern (Textfig. 7). Im distalen Abschnitte der Tentakel, der bei Contraction nach innen zurückgeschlagen ist (Textfig. 3 bei $\ell$ ), liegen breitere, platte Scleriten, daneben auch kleinere, am einen Ende

Fig. 7. I'sculocludochonus. Scleriten aus dem Tentakelriicken mahe der Basis des Tentakels: E von der schmalen Seite gesehen. $\times 335$. $\uparrow$ Sclerit von der breiten Seite, zugespitzte Kalkkörperchen, welche warscheinlich in den Pinnulae liegen (Textfig. 3, bei p, S. 4).

Ein genaueres Eingehen auf die Form und Verteilung dieser Scleriten scheint mir überflüssig und beschränke ich mich auf obige kurze Angaben, welche nur an einigen wenigen Präparaten erhalten sind und deshalb auf grosse Genauigkeit und Vollständigkeit keinen Anspruch machen. Ich fand keine Veranlassung, vom spärlichen Materiale für diesen wenig wichtigen Punkt noch mehr aufzuopfern. 
ZIVEITER TEIL.

\section{Die Verwandtschaft von Pseudocladochonus mit lebenden Alcyonaria.}

$\S 4$. Vergleichung mit den verschiedenen bekannten Typen der lebenden Alcyonaria.

Es ist nicht leicht, dieser neuen Art ihren Platz im Systeme der Alcyonaria anzuweisen. In der Bildungsweise der Kolonien, im eigentümlichen Baue der Stämmchen und in der Art ihrer Verzweigung steht dieselbe ziemlich isolirt da, und man könnte geneigt sein, in Pseudocladochonus einen vollständig neuen Typus der Alcyonaria zu erblicken. Ich glaube aber, dass eine nicht sehr entfernte Verwandtschaft mit dem Genus Telesto besteht, wie weiter unten ausfuhrlicher dargelegt werden soll.

Neben vielen andern Unterschieden trennt das Fehlen einer Hornachse unseren Psendocladochomus von den Gorgoniden sens. strict. (den Holaxonia von Koch's) und von den Pennatuliden. Die ganz andere Bildugsweise des Kalkskeletes und die Verzweigung entfernen die Art weit von den Coenothecalia. Bourne's (Heliopora, etc.).

Die Alcyoniidae, Nephthyidae und Siphonogorgiidac, die Xeniidae, sowie die Psendaxonia zeichnen sich gegenüber Pseudocladochonus aus durch ihr gut entwickeltes Coenenchym, welches die Bases aller Polypen einer Kolonie umgiebt und ein durch die ganze Kolonie continuirlich ausgedehntes Kanalsystem enthält.

Unter den primitiveren kolonienbildenden Alcyonaria, den Stolonifera Hickson's, kommen nur jene vereinzelte Formen in Betracht, bei denen die Polypen, auch in einiger Entfernung vom Boden, aus ihrem Rumpfe noch Stolonen abgeben, aus welchen Stolonen wieder neue Polypen hervorgehen. Wir finden diese Stolonen bei Clavularia viridis Quoy \& Gaim. und bei Tubipora ${ }^{1}$ ); sie gehen in derselben Weise aus den Polypen hervor wie die Stammabschnitte bei Pseudocladochonus. Es bilden aber die Stolonen bei Clavularia viridis und bei Tubipora Querverbindungen zwischen schon gebildeten Polypen, aus welchen Stolonen dann seitwärts, vom Boden abgewendet, neue Polypen hervorwachsen. Alle Polypen einer Kolonie, auch die ältesten, breiten ihre Tentakelkronen so ziemlich in einer Ebene aus; die jiingeren I’olypen

1) Vergleiche: vox Kocu, Anatomic der Orgelkoralle, Jena IS74; Srvxex J. Ilcksox, The structure and relations of Tubipora, Quart. Journ. Micr. Srience, Oct. $1 S_{3}$; Heksox, Alcyonaria Stolonifera, Trans. Zool. Soc. London, vol. I3, prt 9 , ISof. 
erheben sich im allgemeinen nicht iber die zuerst gebildeten. Bei Pseudocladochonus werden dagegen von den Stammabschnitten niemals secundäre Querverbindungen zwischen schon vorhandenen P'olypen gebildet; die neuen Polypen wachsen nicht seitwärts aus den Stammabschnitten hervor, sondern sitzen letzteren terminal auf. Wie ich schon oben S. 6 auseinander gesetzt habe, sehe ich in den Stammabschnitten bei Pseudocladochonus umgeänderte basale Abschnitte der langgestreckten Polypen; die Stammabschnitte sehen zwar den Stolonen von Clavularia viridis sehr ähnlich, sind denselben aber doch durchạus nicht homolog. Als Stolonen resp. Solenia betrachte ich bei Pszudocladochonus nur jene kurzen Gänge welche, indem sie die IVandungen der Polypen durchbrechen, die Magenhöhlen derselben mit den Längskanälen in den Stammabschnitten in Verbindung setzen (Taf. 3, Fig. I I, bei S.). Nach dieser Deutung sind also die Stolonen bei Pseudocladochonus auf einige kurze Kanäle (Solenia) in der IVandung der Polypen beschränkt (vergl. S. 6). Auch darin, dass seine Kolonien verästelte Stämmchen bilden, wobei die Polypen sich sehr verschieden weit vom Boden erheben und die jüngeren Polypen meist die älteren erheblich überragen, weicht Psoudocladochonus wesentlich von den immer mit ihrer Oberfläche mehr parallel dem Boden ausgebreiteten Kolonien von Clavularia viridis und Tubipora ab. Dass die Kolonien von Clavularia viridis sich etwas über den Boden erheben können, verdankt die Art namentlich den Querverbindungen zwischen den parallel sich erhebenden Polypen, daneben auch dem Auftreten eines warscheinlich aus Hornsubstanz bestehenden faserigen Skeletes, welches die Kalkkörperchen mit einander verbindet. Bei Tubipora wird eine viel grössere Festigkeit dadurch erreicht, das neben einer Verbindung aller Polypen mittelst der plattenformigen, horizontalen Stolonen, auch die Kalkkörperchen zu einem zusammenhängenden, oberflächlichen, die Weichteile umgebenden Skelete fest verbunden sind. Ein ähnlicher Zusammenhang der Kalkkörperchen giebt auch den Kolonien des Pseudocladochonus die erforderliche Festigkeit und ist hier um so bedeutungsvoller als die Stämmchen sich gänzlich frei über den Boden erheben ohne von benachbarten Stämmchen mittelst querer Stolonen gestützt zu werden.

Auf dem Wege, welcher zur Bildung einer vom Boden emporwachsenden, verästelten Kolonie fuhrt, hat sowohl in der Verzweigung wie im Skelete die Clavularia viridis nur erst cine viel niedrigere Entwicklungsstufe erreicht als Pseudocladochonus. Dabei hat Clavularia viridis in der kräftigen Entwicklung des Hornfaserskeletes ${ }^{1}$ ) in der Nesogloea einen eigenen Weg eingeschlagen. Ich kann nur eine sehr entfernte Verwandtschaft dieser Art mit Pscudocladochonus annehmen. Insoweit bei beiden Arten die Polypen in einiger Entfernung vom Boden noch Stolonen (resp. Solenia) bilden, aus denen neue Polypen hervorgehen, besteht Ähnlichkeit, aber dies ist auch der einzige Punkt, worin sie übereinstimmen. Und dabei ist Convergenz nicht auszuschliessen, zumal wenn wir die abweichende Ausbildung des Skeletes bei Clavularia viridis mit in Betracht ziehen.

Mit Tubipora hat Pseudocladochonus nicht nur die seitwärts an den Polypen gebildeten

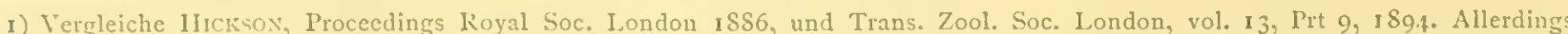
finde ich auch in einigen meiner Präparate von I'seudoclatochonus verïstelte Fasem, welche aus Hornsubstanz bestehen könnten. I)ieselben sind nur seht wenig zahlreich und liegen ganz oberflächlich; sie gehören vielleicht der Cuticula der Epidermis an, welche ja bei Alcyonaria bisweilen deutlich entwickelt ist (vergl. vox Kocii, Morphol. Jahrb., IBd 5, IS79, p. 320 und IBd 7, ISS2, p. 472). 1)ieses Hornfasershelet crreicht aber jedenfalls nicht die grosse liedeutung, welche es bei Chadbaria siridis besitzt. 
Stolonen, sondern auch das aus verschmolzenen Scleriten bestehende, die Weichteile umhüllende Skelet gemeinsam. In der Ausbildung der Stolonen aber und im Aufbau der Kolonien überhaupt ist Tubipora von Pseudocladochomus doch recht verschicden. Ich betone nochmals die zahlreichen, einander parallelen Polypenröhren mit den dieselben in verschiedener Höhe verbindenden, plattenartigen Stolonen bei Tubipora, wogegen Pseudocladochonus baumförmig verästelte, cinen deutlichen Stamm besitzende Kolonien bildet, bei welchen die Stolonen auf einigen Solenia in der Wandung der Polypen beschränkt bleiben. Diese Unterschiede sind so wichtig, dass ich auch hier keine enge Verwandtschaft anzunehmen vermag. Pseudocladochonus muss selbstverständlich von den Stolonifera abstammen; dass die Entwicklung diestr Form ausgegangen ist von Arten, welche mit Clavularia viridis und Tubipora verwandt waren, ist nicht unwarscheinlich, betrachte ich aber nicht als gesichert.

\section{Vergleichung von Pseudocladochonus mit den Telestidae.}

Wie Pseudocladochonus bilden auch die Telestidae verästelte Kolonien, bei welchen die Polypen nicht in einer, allen gemeinsamen Coenenchymmasse eingebettet sind: auch haben Pseudocladochonus und die Telestidae die Besonderheit gemeinsam, dass die neuen Polypen hervorwachsen aus Kanälen, welche in der Wandung der alten Polypen liegen (vergleiche Taf. 3 , Fig. I I und 12). Letzteres trifft nur vollständig zu, wenn man in den Stammabschnitten des Pseudocladochonus (Taf. 3, Fig. I I, A, St.) die umgeänderten basalen Abschnitte von Polypen sieht, welche sehr in die Länge gewachsen sind (vergl. S. 6). Nimmt man diese Homologie an, so wird die. Ähnlichkeit mit den Telestidae eine sehr grosse, wie bei einer Vergleichung der Figuren I I und I 2 auf Tafel 3 deutlich hervortritt. Pseudocladochonus hat dann mit den Telestidae folgendes gemeinsam: I Die Polypen strecken sich in die Länge und bilden die Stämme und Äste; $2^{n}$ die neuen Polypen wachsen aus der Körperwandung eines anderen Polypen hervor, wobei die Verbindung der Magenhöhle des neuen Polypen mit der Magenhöhle des Mutterpolypen von kurzen Kanälen hergestellt wird, welche den Solenia anderer Alcyonacca entsprechen, hier aber meist für jeden Polypen ein gesondertes System von Kanälen bilden (Taf. 3, Fig. I I und I 2, S.). Ein festes, aus verschmolzenen Scleriten bestehendes Skelet kommt auch einigen Telesto-Arten zu, z.B. Telesto trichostemma und mbra ${ }^{1}$ ). Die von Hicksor") gegebene Abbildung eines Skeletfragmentes der letzteren Art sieht dem Skelete des Psezdocladochonus sehr ähnlich und eigene Untersuchung von Telcsto-Arten aus dem Materiale der Siboga-Sammlung hat diese Ähnlichkeit bestätigt; die Kalkkörperchen sind bei einigen TclestoArten vom selben Typus, wie bei Pscudocladochonus, oft auch mit gegabelten Enden; bei anderen Tclesto-Arten sind sie allerdings mehr nadelförmig. Die Art wie die Tentakelkrone samt dem distalen Polypenabschnitte (Bourne's Anthocodia) in den basalen Polypenkelch zuruickgezogen ist, ist bei beiden Genera vollständig iclentisch, wie aus den Schemata (Taf. 3, Fig. I I und 12)

1) Vergleiche auch: von Kocir, Morphol. Jahrb., 13d 7, ISSz, laf. 22, Fig. 19c; und S. 474.

2) The Alcyonaria of the Maldives, l'nt I; liama and Geography of the Maldive and Laccadive Archipelagoes, vol. 2, Prt I, I 903 , Nlate 26, Fig. 2. 
ersichtlich ist. Man vergleiche auch meine Textfiguren 2 und 3 (S. 3 und 4) mit von Kocri's Figur 7 , Tafel 22, Morphol. Jahrb. Bd 7, r 882 . Wegen der Unterschiede, welche bis jetzt gefunden worden sind in der Art, in welcher die Polypen ihre Tentakelkrone zurückziehen, glaube ich dieser Übereinstimmung hier einigen Werth beimessen zu müssen, wiewohl sowohl unsere ungenügende Kenntnis über diesen Punkt, als auch das Vorkommen desselben Retractionszustandes bei anderen Alcyonaria ${ }^{2}$ ), es verbieten, denselben als von grosser Wichtigkeit zu betrachten.

Wegen dieser Ähnlichkeiten glaube ich Pseudocladochonus als einen Telestiden betrachten zu müssen. Namentlich die Art, in welcher die neuen Polypen aus der Wandung der älteren hervorknospen, ist fur mich in dieser Frage entscheidend.

Es hat sich aber Pseudocladochonus in einer, von den ubrigen Telestidae (Telesto, Pseudogorgia, Coclogorgia $\left.{ }^{2}\right)$ ) abweichenden Weise weiter entwickelt. Das Skelet erstreckt sich auch in die Mesenterien, vor allem im basalen Polypenabschnitte. Die Mesenterien gewannen dadurch Bedeutung für die Festigkeit der Kolonien; sie traten im basalen Abschnitte der Polypen mit einander in Verbindung. Damit wurde eine Sonderung des Polypenkörpers in einen kurzen distalen, normal gebauten Abschnitt und einen langen basalen Stammabschnitt hergestellt. Neue Polypen knospen nur noch aus dem distalen Abschnitte hervor, der kurz ist und dementsprechend nur eine oder zwei Knospen bildet, während bei den übrigen Telestiden die langen Mutterpolypen, welche die Stämme und Äste bilden, eine grössere Zahl von Polypen tragen können. Bei Pseudocladochonus sind alle Polypen einander gleich; es fehlt jener Unterschied zwischen Achsen- oder Nutterpolypen und Seitenpolypen, der für Telesto, Coclogorgia und Psendogorgia so typisch ist. Die Stämme von Telesto scheinen nur terminal in die Länge zu wachsen; demgegenüber zeichnet Psezdocladochomus sich warscheinlich aus durch ein secundär erworbenes, intercalares Längenwachstum der Stammabschnitte (vergl. S. 9). Ob bei Pseudocladochonus auch basale, ïber dem Boden kriechende Stolonen entwickelt werden, wie sie bei Telesto und Coclogorgia auftreten, darüber giebt mein Naterial keine Auskunft.

Die Beziehungen von Pserdocladochonus zu den Telestidae sind meines Erachtens ziemlich deutlich und zögere ich nicht, das Genus in dieser Familie unterzubringen.

Doch muss dann die übliche Diagnose der Familie Telestidae, wie sie z.B. sehr klar von $\left.\bigwedge_{A Y}{ }^{3}\right)$ gegeben worden ist, in einem wesentlichen Punkte umgeändert werden. MAY's Diagnose legt nämlich Gewicht darauf, dass die Kolonien der Telestidae aus aufrechten Achsenpolypen und lateralen Polypen zweiter bis dritter Ordnung bestehen, und auch Bourne ${ }^{4}$ ), Delage-Hérouard ${ }^{5}$ ) und Hicksox ${ }^{B}$ ) haben dies besonders hervorgehoben. In diesem Punkte weicht aber Pseudo-

I) z.B. bei Alcyonium: sehr deutlich ist dies abgebildet anf einer Figur, die IIckson gegeben hat (The Anatomy of Alcyonium digitatum, Quart. Journ. Micr. Science, Vol. 37, N.S. 1S95, P1. 3S, Fig. 13).

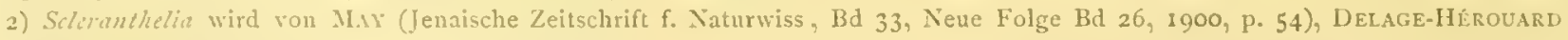
(Zoologie Concrète, Coelentérés, Igor, 1\% 399) und Kükexthtal (Wiss. Ergebnisse der Deutschen Tiefsee-Exped. Valdivia, Bd I3, Lief. I, Alcyonacen, 1906, p. 90) zu der Familie Tilistilai gerechnet. Ich habe von der Type dieser seltenen Art ein Fragment gesehen, welches aus der Sammlung von PERCrAL WrigrT in den Besitz der Victoria Universität in Manchester übergegangen ist, und stimme STUDER bei, wenn er (Résultats des Campagnes Scientif. Monaco, Fasc. 20, Alcyonaires, 19or, p. I3) das Genus zu den Clazularỉlae rechnet. Es scheint mich recht fraglich, ob irgend welche Beziehungen zu den Tilistita bestehen, obwoll das Genus vielleicht die Art andeutet, in welcher die knospung der Tilistidai sich entwickelt haben kann.

3) Jenaische Zeitschrift fir Naturwiss., Bd 33, Neue Folge 13d 26, 1900, p. 54.

4) Treatise on Zoology, edited by lay I.ankester, Prt 2, Anthozoa, 1900, p. 29.

5) Zoologie Concrète, Coelentérés, 1901, p. $39 \mathrm{~S}$.

6) Coelenterata, lihe Cambridge Natural History, Vol. I, Igo6, p. 345. 
cladochonus von allen andern Telestidae ab; bei ihm sind alle Polypen gleich; jeder bildet einen Abschnitt der Stämmchen, welche letztere sympodial gebaut sind; ein Gegensatz von Seitenpolypen und axialen, stammbildenden Polypen besteht bei unserm neuen Genus nicht.

Ich schlage also folgende Diagnose der Familie Telestidae vor: Die Kolonien erheben sich vom Boden. Die Stämme und Äste resp. Stammabschnitte werden dabei nur von je einem langgestreckten Polypen gebildet. Aus der Wandung der Polypen knospen neue Polypen hervor, welche nur mit der Basis ihrem Mutterpolypen angeheftet sind. Die Verbindung der Höhlungen der Polypen mit ihren Mutterpolypen wird von kurzen, die Polypenwandung durchsetzenden Kanälen hergestellt, welche den Stolonenkanälen oder Solenia anderer Alcyonaria entsprechen. Die Solenia sind also nicht auf die Basis der Polypen beschränkt, sondern gehen auch höher aufwärts aus der Magenhöhle der Polypen hervor. Das Skelet besteht aus Kalkkörperchen, welche in der Mesogloea liegen und welche zu einem festen Skelete verschmelzen können.

$\mathrm{Zu}$ den Tclestidae rechne ich die Genera Tclesto, Coclogorgia, Pseudogorgia und Pseudocladochonus. Scleranthelia bringe ich nicht dazu (vergleiche die Anmerkung S. 14), sondern lasse dieses Genus mit STUder in der Familie Clavnlariidae.

Die recht abweichende Telesto arthuri Hickson und Hiles ${ }^{1}$ ) glaube ich nach eigener Vergleichung der Type, welche mir durch das sehr freundliche Entgegenkommen Professor Hickson's möglich war, nicht zu den Telestidae rechnen zu dürfen. Ich sehe darin einen Vephthyiden oder einen Siphonogorgiiden.

Herrn Professor Hickson verdanke ich es gleichfalls, dass ich eine sehr werthrolle Querschnitt-Serie durch einen axialen Polypen mit Seitenpolypen von Telesto mura, welche sich in der Sammlung der Victoria Universität (Owen's College) zu Manchester befindet, habe studiren können. Zum weiteren Vergleiche diente mir ein schönes Material von Coelogorgia und von mehreren Telesto-Arten, welches die Siboga-Expedition im. Ost-Indischen Archipel gesammelt hat.

S6. Diagnose des neuen Genus Pseudocladochonus.

Die Polypen sind einander alle gleich; jeder hat Anteil an der Bildung der Stämmchen und jeder ist gesondert in einen längeren basalen Stammabschnitt und einen kurzen distalen Kelchabschnitt, in welch letzteren die Tentakel samt der Mundscheibe der Polypen bei Contraction zurückgezogen sind. Neue Polypen gehen nur aus dem Kelchabschnitte hervor und zwar nur einer oder zwei, niemals mehrere, aus jedem Polypen. Im Stammabschnitte der Polypen sind die acht Mesenterien in der Längsachse mit einander verwachsen und ist dadurch die Magenhöhle in \& Längskanäle gesondert. Das Skelet des Stamm- und Kelchabschnittes besteht aus in einer Schicht liegenden, fest mit einander verwachsenen Scleriten, welche eine nur von kleineren Löchern durchbohrte Skeletplatte bilden. Dieses Skelet findet man auch in den Scheidewänden (Mesenterien) zwischen den Längskanälen der Stammabschnitte und in der centralen Längsachse, wo diese Scheidewände zusammentreffen.

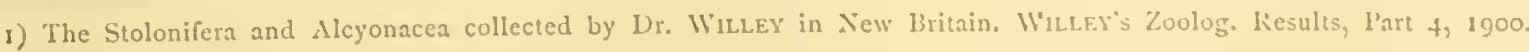


Mit nur einer Art: Pseudocladochonus hicksoni. Zarte Kolonien, in Alcohol von weisser Farbe, mit deutlichen Biegungslinien, welche etwas gelblich sind.

Für weitere Besonderheiten sei auf die ausführliche Beschreibung weiter oben verwiesen.

7. Beziehungen der Telestidae zu den Gorgoniden.

Es ist nicht meine Absicht, schon hier auf die von Studer ${ }^{1}$ ) versuchte Ableitung der Gorgoniden von Telestiden, an welche Hypothese sich auch Menneking ${ }^{2}$ ) und KÜKEnThaL ${ }^{3}$ ) angeschlossen haben, näher einzugehen. Aber einige Bemerkungen über diese Frage seien hier aufgenommen, namentlich weil Pseudocladochonus in seinem Bau für die STUder'sche Ansicht sehr wichtig ist.

Bekanntlich hat Studer ${ }^{4}$ ) die Hypothese aufgestellt, dass die Stämme und Äste der Gorgonidcn sich entwickelt haben aus axialen, langgestreckten Polypen, wie Tclesto und Coelogorgia sic aufweisen. Er muss hierfiir annehmen, dass die 8 Mesenterien der Alcyonaricr-Polypen, welche bei den Stammpolypen der Telestiden sehr in die Länge gezogen sein können ${ }^{5}$ ), bei den Gorgoniden in der Achse der Magenhöhle zu einer centralen Säule verschmolzen sind; dadurch wird dann die bei Telesto einheitliche Magenhöhle in 8 geräumige Längskanäle gesondert, welche die Hauptkanäle in der Rinde der Gorgoniden-Stämme bilden. Es ist nun sehr interessant und fiir Studer's Hypothese sehr wichtig, dass bei Pseudocladochomus diese vorausgesetzte, aber bis jetzt noch von keinem Telestiden bekannte Sonderung der Magenhöhle der Polypen in 8 Längskanäle durch Verschmelzung der Mesenterien in der Achse der langgestreckten Polypenbasis statt gefunden hat. Von einem andern Telestiden, Pseudogorgia, war zwar schon eine Verschmelzung von Mesenterien bekannt, aber nur von 4 derselben zu zwei Scheidewände, die nicht mit einander verbunden sind $\left.{ }^{6}\right)$. Sowohl in dieser Verschmelzung der Mesenterien wie in der damit zusammengehenden scharfen Sonderung der Polypen in einen distalen, nicht umgeänderten Abschnitt und einen langen, basalen Stammabschnitt geht Pseudocladochonus auf dem von Stuner postulirten Entwicklungswege der Gorgoniden (Holaxonia) weiter, als jeder andere Telestidc. Auch dass bei diesen Stammabschnitten ein intercalares Längenwachstum aufzutreten scheint, möchte ich hervorheben, da dieses auch bei der Rinde der Gorgoniden stattfindet, namentlich deutlich bei den Primnoidae, welche STUder wegen ihres sehr einfachen Kanalsystems der Rinde bei der Aufstellung seiner Hypothese besonders herangezogen hat.

Psendocladochonzes hat in Übrigen, so namentlich im sympodialen Aufbau seiner Stämmchen, einen andern $\mathrm{W}$ eg eingeschlagen, als die von Studer gedachten Stammformen der Gorgoniden und kommt dadurch als solche nicht selbst in Betracht. Sein Bau beweist aber, dass auch bei einer Form wie Telesto eine Verschmelzung der Mesenterien und die Sonderung der

I) Archiv fur Naturgeschichte, Jahrg. 53, Bd I, $1 \$ 87$, p. 5, 6, 35.

2) Archiv fur Naturgeschichte, Jahrg. 71, Bd I, 1905, P. 247.

3) Wiss. Ergebnisse der Ineutschen Ticfsec-Lixp. Valdivia, Bd 13, Lief. I Alcyonacea, 1906, p. 90, $98,99$.

4) 1. c.; vergleiche auch: Wrigut and STUsk:, Challenger leport, vol. 3i, isSg.

5) z.1\%. hei Tilisto (Carijoa) rupicola F. Miller, Archiv. f. Naturgesch., Jhrg. 33, Ijd I, IS67, p. 33I, und bei Tilisto arborca,

IVRTinT and STUDFR, Challenger Keport, vol. $31,1 S S 9$, p. 260 and 263.

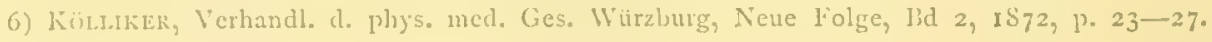


Magenhöhle der axialen Polypen in \& Längskanälen sehr gut hat stattfinden können. Er liefert also der Studer'schen Hypothese eine sehr wesentliche Stutze.

Dennoch muss ich gestehen, dass mir die STUder'sche Hypothese damit noch nicht genügend begründet erscheint. Die Gorgoniden, auch die Primnoidae, deren Kanalsystem neuerdings mit Riicksicht auf die Studer'sche Hypothese von Mexweking untersucht wurde, tragen, soweit mir bekannt, niemals typisch terminale Polypen an den Spitzen der Zweige, welche den Stammkanälen genau so aufsitzen wie bei Pscudocladochonzs die distalen, nicht umgeänderten Abschnitte der Polypen (Taf. 3, Fig. 1 1, K.) den Längskanälen der Stammabschnitte (Taf. 3, Fig. I I, St.). Oft ragen bei den Gorgoniden, namentlich auch bei den Primnoidae, die Spitzen der Zweige etwas über die am meisten benachbarten Polypen vor. Auch die Endpolypen, welche bisweilen auf den Zweigspitzen von Thouarella hilgendor $f \imath$ u. a. Arten sitzen, stehen immer etwas schräg ${ }^{1}$ ). VON Kocri's Untersuchungen ${ }^{2}$ ) über die ersten Entwicklungsstadien der GorgonidenKolonien haben auch ergeben, dass die Achse nicht in Centrum der Magenhöhle des erstgebildeten Polypen entsteht, sondern der einen Seite dieses Polypen deutlich genähert ist.

Die Achtzahl der Hauptkanäle in der Rinde der Primnoidae ist durchaus nicht constant und es ist weder von Stuner noch von Menneking angegeben, weshalb sie glauben diese Zahl, wo sie bei den Gorgoniden auftritt, als primitiv betrachten zu diurfen.

Auch stehen bei den Telestidae die Magenhöhlen der Seitenpolypen immer nur mittels Solenia in Verbindung mit der Magenhöhle der Mutterpolypen aus denen sie sich entwickelt haben. Bei den Gorgoniden dagegen können die Magenhöhlen der Polypen unmittelbar übergehen in die geräumigen Rindenkanäle ${ }^{3}$ ), welche nach Studer den Nagenhöhlen der Mutterpolypen der Telestidae entsprechen. Dies deutet aber darauf hin, dass die Rindenkanäle der Gorgoniden selbst Solenia sind.

Die Bildung einer Hornachse mit Achsenepithel bei den Gorgonidcn ist ein weiterer sehr erheblicher Unterschied gegeniiber den Telestiden. Ich kann mich der StudeR'schen Ableitung der Gorgoniden von Telestiden denn auch nicht anschliessen. Eine nähere Begründung dieser Ableitung von Studer oder von Kürenthat, der eine ähnliche Auffassung vertritt, bleibt aber abzuwarten.

1) Verglciche Veksqurs, Primnoidae, Siboga-Expeditic, Monographic 13 m, Leiden, 1906, 1. $14 \mathbf{1}$.

2) Die Gorgoniden; Fauna und Flora des Golfes von Neapel, Berlin ISS7; vorliufige Mitteilungen in: MIttcil. Zoulum. Siation zu Neapel, Bad 3, ISS2, p. 550, Fig. 14.

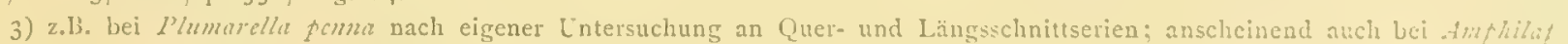
nlictina nach der Fig. 20, Taf. 9 von MesiekisG, Archiv f. Naturgeschichte, Jahig. 7x, Bd I, 1905. 


\section{DRITTER TEIL.}

\section{Die Verwandtschaft von Pseudocladochonus mit dem fossilen Genus Cladochonus M'Coy}

\section{S. E. Enleitende Bemerkungen; Material.}

Das Genus Psendocladochomus zeigt eine auffallend grosse Ähnlichkeit mit dem carbonischen Genus Cladochonus M'Coy. Schon eine Vergleichung der Figur 10, namentlich ro C, auf Taf. 3 mit den Textfiguren 8 (S. 20), I I A (S. 21) und I 2 (S. 2 I) giebt Anlass zur Frage, ob wir es hier nicht mit sehr nahe verwandten Formen zu thun haben. Es scheint ein näheres Eingehen auf die Frage, ob diese Ähnlichkeit ein Zeichen von Verwandtschaft ist oder lediglich auf Convergenz beruht, geboten. Dazu kommt noch, dass von einigen Untersuchern, wie DANA ${ }^{2}$ ): früher auch HAECKEL $^{2}$ ), und von SARDESON $^{3}$ ), die Auloporidae, zu welcher Familie Cladochonus gehört, bereits zu den Alcyonaria gebracht worden sind. H. A. Nicholson ${ }^{4}$ ) betrachtet diese Zugehörigkeit zu den Alcyonaria als warscheinlich, wenn auch durchaus nicht als gesichert. Delage-Hérouard ${ }^{5}$ ) fuihren die Auloporidae mit einigem Zweifel auch unter den Alcyonaria bei den Tubiporinae auf. K. A. vor Z ITTEL $^{6}$ ) hat anfänglich, allerdings unter Vorbehalt, die Auloporidae auch zu den Alcyonaria gerechnet, sie aber später ${ }^{7}$ ) wieder davon abgetrennt und zu den Tabulata gebracht. HaEckel führt sie in seiner "Systematischen Phylogenie" ebenfalls nicht mehr unter den Alcyonaria auf, sondern bei den Tabulata (1. c., p. 203).

Es schien im Anfang meiner Untersuchung möglich, dass die vermutete Verwandtschaft der Auloporidae mit den Alcyonaria, durch die neue Siboga-Form eine Bestätigung finden würde. Damit wäre dann die Verwandtschaft einer eigentümlichen, abweichenden Gruppe fossiler Korallen ${ }^{\triangleright}$ ) mit lebenden Formen aufgeklärt worden. Die nähere Untersuchung von Cladochonus hat aber ergeben, dass die Ähnlichkeit mit Pseudocladochonus wohl nur auf Convergenz beruht und es bleibt damit die Verwandtschaft der Auloporidae so dunkel wie vorher. Die

1) Zooplyytes, Lnited States Exploring Expedition, 1848 .

2) Dies wird von Delagl-Hérouard in ihrer ,Loologie Concrète" (Coelentérés, Igor, p. 392) angegeben. Mir ist die Stelle bei IAECKEI, selbst nicht bekannt.

3) Neues Jahrbuch fur Mineralogie, Geologie und Palaeontologie, Bd Io, Beilage Band, ISg6, p. 249.

4) Palacozoic Tabulate Corals, London IS79.

5) l. c., P. 39r, 392 .

6) Handbuch der Palaeontologic, Bd I, IS76-ISSO, P. 2Io-2II.

7) Grundzüge der Palaeontologie, 1 S95, p. 94. den Irryozoz.

S) Dass die Auloperidu' liomllen sind, ist noch nicht über jeden Zweifel erhaben; es besteht auch einige Alunlichkeit mit 
Gründe, auf welchen diese Schlussfolgerung beruht, habe ich dennoch ausführlich geben wollen, da ich bei der sehr grossen Ähnlichkeit von Pseudocladochonus mit Cladochonus meine Ansicht, dass die neue Siboga-Art nicht zu den Auloporidac gehört, notwendig näher begriinden muss.

Zur Beantwortung dieser Frage habe ich vor allem das Genus Cladochonus selbst untersucht, habe aber auch verwandte Genera in den Kreis meiner Betrachtungen gezogen, und zwar Aulopora, Monilopora, Ceratopora und auch Syringopora. Letzteres Genus zeigt nämlich nicht nur Beziehungen zu Aulopora, sondern auch vor allem zu Ceratopora Grabau. Die jungen Kolonien von Syringopora sind Aulopora sehr ähnlich; so sagt CHapan ${ }^{3}$ ) über Syringopora serpens: "The specimens from Gotland are small, detached corallites of the early stages of Syringopora. They are remarkably like Aulopora and Cladochonus in general appearance; and this bears out Messrs Enwards and Haime's statement that „young specimens of this coral equally resemble Aulopora".". Mit Ceratopora hat Syringopora den sehr typischen, eigenthiumlichen Bau der Wandungen der Polypenkelche gemeinsam. Man vergleiche dazu Grabau's Beschreibungen und Abbildungen von Ceratopora ${ }^{2}$ ) mit den von Niciolsos gegebenen Abbildungen von Schliffpräparaten durch Syringopora ${ }^{3}$ ). Nur wenn es sich herausgestellt hätte, dass der Bau aller dieser Genera sich mit einer engen Verwandtschaft von Pseudocladochomus mit Cladochonus in Einklang bringen liess, hätte man diese Verwandtschaft als gesichert betrachten können. Dies ist meines Erachtens nicht der Fall.

Ich verfügte für meine Untersuchung über einiges Material von Cladochomus michclini Edw. \& Haime, aus Tournai, Belgien, und über ein Gesteinsfragment welches grösstenteils aus Fragmenten einer nicht näher bestimmten Cladochonus-Art aus dem Carbon von Button Mould Knob, Kentucky; U.S. A. besteht. Ich verdanke das Material von Cladochonus michelini den Bemühungen von Herrn Professor Dr. E. Dubors in Haarlem; die andere Cladochonzu-Art wurde mir geschenkt von Herrn Dr. G. J. Hinde, South Croydon, London, der dieselbe selbst gesammelt hatte. Daneben verfuigte ich uber einige gut erhaltene Exemplare von Aulopora.

Während eines Besuches in London habe ich auch das Material von Auloporidac im British Museum of Natural History vergleichen können; darunter befindet sich ein schönes Material von Monilopora crassa M'Coy. Ich fand darunter auch die Schliffpräparate von Mlonilopora crassa, welche Nicholson und Etheridge beschrieben und abgebildet haben ${ }^{*}$ ) (British. MIus. Nat. Hist. $N^{10} 90234$ und 90235); ich habe diese Präparate selbst studiren können. Sehr wichtige Huilfe habe ich von Dr. G. J. HrNne empfangen. Nicht nur hat er mir die oben erwähnte Cladochonus species geschenkt, sondern er hat mir das vollständige diesbeziigliche Material aus seiner Privat-Sammlung vorgelegt und mich auch bei Herrn Dr. F. L. Kitcrin vom "Geological Survey of the United Kingdom" eingeführt, der mir in freundlichster Weise die genauere Durchsicht der im Museum des Geological Survey, Jermynstreet, London, vorhandenen Auloporidac gestattet hat. Das Wichtigste darunter war für mich Material von Cladochomus bacillarizs M'Coy

1) Annals and Magazine of Natural History, $7^{\text {th }}$ ser., vol. 7 , rgor, p. 143-144.

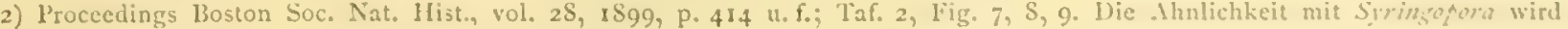
von Grabau nicht erwähnt.

3) Palaeozoic Tabulate Corals, London, $1 S_{79}$, "Taf. 10, Fig. 5 und 'Texthig. 30 A und 13, p. 211.

4) Geological Magazine, New Series, Decade 2, vol. 6, 1\$79, p. $2 \$ 9,296$, I'l. 7 , tig, 2 I), 2 E und I F. 
(Kohlenkalk Narrowdale), Cl. campanulatıs M'Coy (Kohlenkalk Northumberland); Cl. species (Kohlenkalk, Button Mould Knob, Kentucky) und Cl. species (von Keokuk, Jowa), letztere beiden von Dr. Hixde gesammelt. Dann Aulopora cormeta? Billings, A. tubacformis? Goldfuss und $A$. canadensis Nicholson (alle drei von Arkona, Ontario, Hamilton Formation, Devon); A. filiformis Billings (Hamilton Formation, Devon, von Thedford, Ontario) und Romingeria umbellifera Billings (Devon, Port Colborne, Lake Erie, Ontario, Canada). Auch einige Dünnschliffe standen mir zur Verfügung (vergl. Ş I I).

\section{S9. Die wichtigsten Beschreibungen und Diagnosen des Genus Cladochonus M'Coy.}

Bei der Aufstellung des Genus Cladochom hat M'Cov dasselbe wie folgt beschrieben ${ }^{1}$ ):

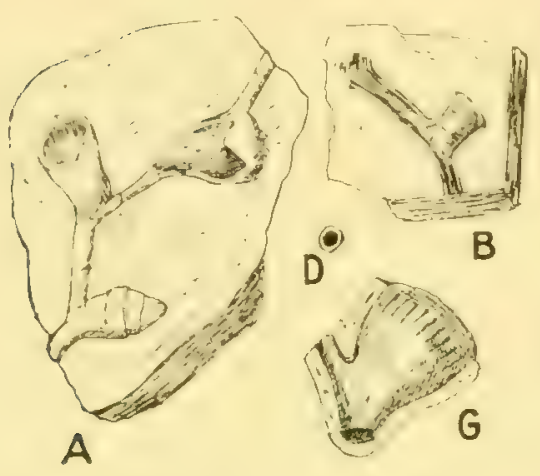

Fig. 8. Clatiochonus timuicollis.

Copie nach M'Cor; Annals and Mag. Nat. Hist., vol. 20, 1847 , Taf. II, Fig. 8 ;

A Fragment einer Kolonie; B ein der Iänge nach gespaltenes Fragment; C Polypenkelch von innen, mit "septal striae"; D Querschnitt durch den Stamm. Alle Figuren vergrössert.

„Gen. Char.: Polypidom of very thick, straight, slender, calcareous tubes, suddenly dilating at short regular distances into large, oblique, cup-shaped terminal chambers, longitudinally striated within; from the point where the dilatation commences, a second slender tube similar to the first is given off at an angle, varying with the species, and terminating at the same distance as the. former in a similar cup, bent in nearly an opposite direction to the first, and giving rise at its base to a third slender tube as before. The whole polypidom erect, attached by the base only, which embraces some foreign body". Dann heisst es weiter: "These singular and beautiful corals have some relation to Aulopora, but differ in their curious erect habit, regular, angular mode of branching, slender, equal, stem-like tubes and abruptly dilated terminal cups bent in nearly opposite directions".

Von seiner Abbildung gebe ich hier eine Copie in Textfigur 8.

Ausfuihrlich ist auch die Beschreibung, welche M. L. G. DE Koninck von Cladochonus gegeben hat ${ }^{2}$ ). Seine Diagnose des Genus ist wie folgt: „Polypier composé de polypiérites non rampants, se multipliant par gemmation latérale, ayant la forme d'un cornet muni d'un pédicule cylindrique de longueur variable, et entourés d'une forte épithèque. Calice circulaire assez profond, à surface interne garnie de faible stries cloisonnaires. Plancher nul". Cladochonus michelini wird dann weiter von DE Koxinck wie folgt beschrieben ${ }^{3}$ ): "Cladochonus michelini (Pyrgia michelini M. Edw. et Haime I 851). Polypier composé d'une série de polypiérites qui, lorsqu'ils sont isolés, rappellent assez bien la

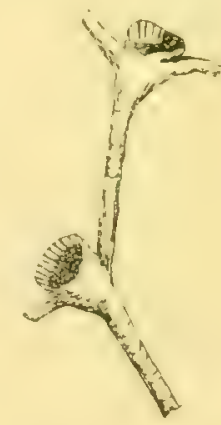

Fig. 9 . Claduchonus michelini. Copie nach vE Koxixck, Mém. Acad, Roy. Belg., 1. 39. I\$72. Tal. I5 Fig. 6. Ein Fragment; vergrössert. forme d'une pipe ordinaire et prennent généralement naissance les uns sur les autres, vers la

1) The Annals and Magazine of Natural History, vol. 20, 1847, p. 227.

2) Mémoires de l'Académie Koyale des Sciences, des Lettres et des Beaux-Arts de Belgique, 'Tome 39, IS72, p. 150.

: 1. . . 1. 153 
base de leur calice, où ils sont légèrement courbés, et se prolongent en un long pédicelle droit et subulé. L'insertion est souvent isoléee, mais il n'est pas rare non plus d'en observer deux et même trois sur le même individu; lorsqu'il y en a deux, elle est opposée l'une à l'autre et divergente; la troisième, lorsqu'elle existe, est située plus bas et, dans ce cas, le calice forme un cone plus allongé. L'épithèque est forte et ne laisse apercevoir que de faibles stries d'accroissement. Tous les polypiérites sont creux et communiquent librement les uns avec les autres. Vers la moitié de la longueur des calices, on observe des stries cloisonnaires, courtes et peu apparantes, au nombre de vingt environ. Longueur des polypiérites,

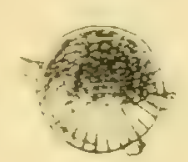

Fig. 10.

Cladochonus michelini. Copie nach IE KON1sck, 1.c. Fig.6r. Polypenkelch von oben gesehen, vergrössert, um die ${ }_{n}$ strie cloisonnaires" zu zeigen. I 2 à 14 millimètres; diamètre de leur calice, 3 à 4 millimètres, et du pédicelle, I millimètre". De Koninck's Figuren 6 und $6 a$, Taf. I5, habe ich hier copirt in den Textfiguren 9 und 10. Nicholson und Etheridge ${ }^{1}$ ) haben dann noch eine ausfuhrlichere Mitteilung uber das Genus Cladochonzs veröffentlicht. Das wichtigste Ergebniss ihrer Untersuchung ist die Abtrennung einer Art, Cl.crassa, vom Genus Cladochonus; für diese Art wird wegen der abweichenden Structur der Wandung das neue Genus Monilopora gegründet. Für den Bau des Genus Cladochonus ist von Bedeutung, dass diese Untersucher für Cl. michelini das bisweilige Auftreten von Tabulae angeben und dieselben beim verwandten Genus Aulopora nachweisen.

Die Resultate dieser Untersuchung von Nicholsox und Etheridge sind aufgenommen in Nicholson's bekannter Arbeit: On the Structure and Affinities of the Tabulate Corals of the Palaeozoic Period, I879, p. 219.

SIO. Vergleichung des Baues von Pseudocladochonus und Cladochonus.

Es sei bei dieser Vergleichung vor Allem auf den erstaunend ähnlichen Habitus hingewiesen. Das ist aus den Abbildungen und Beschreibungen von M'Coy und DE Konrixck ersichtlich (vergl. ร 9). Die Diagnose, welche M'Coy vom Genus Cladochomus gegeben hat, passt

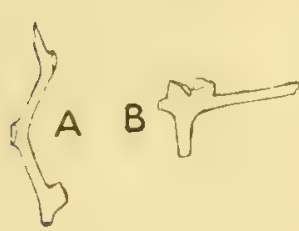

Fig. 22. Cladochonus michclini. Zwei liragmente in wahrer Grüsse. I Fragment mit 3 Kelchen, nicht verzweigt; I3 ein Kelch, der 2 Stämmchen abgiebt. auch nahezu vollständig fiur Pseudocladochonus (vergl. oben S. 20). Ind auch die Vergleichung von Pseudocladochonus mit meinem eigenen Materiale von Cladochonus, sowie mit demjenigen, welches die Sammlungen des Herrn Dr. Hivde und des, Museum of the Geological Survey of the United Kingdom" in Jermynstreet, London, enthalten, hat diese Ahnlichkeit vollständig bestätigt. Man vergleiche namentlich "lextfig. I A mit Fig. IoC auf Taf. 3. Die in den Textfiguren 9, I I und I 2 abgebildeten Fragmente zeigen uns, wie die Aste von Cladochonus michclini etwa ausgesehen haben und aus meinem

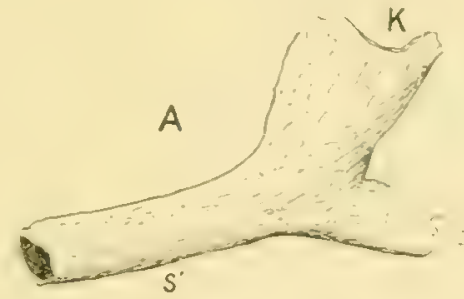

$s^{\prime}$

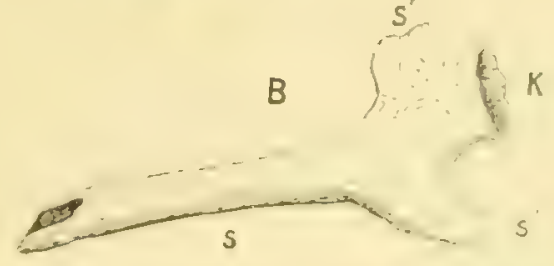

Fig. II. Chutocionus michlini; Fragmente. $\Delta$ Kelch, der einen stamm abciebt ( $S^{\prime}$ rechts): $B$ Kelch, der zwei Stämehen $\left(S^{\prime}\right)$ abgielst: $K$ Polypenkelch, $S, S^{\prime}$ Stammabchnitte; $>$ ' 
Materiale, sowic aus der Beschreibung von DE Koninck kann man auf einen Bau dieser Art

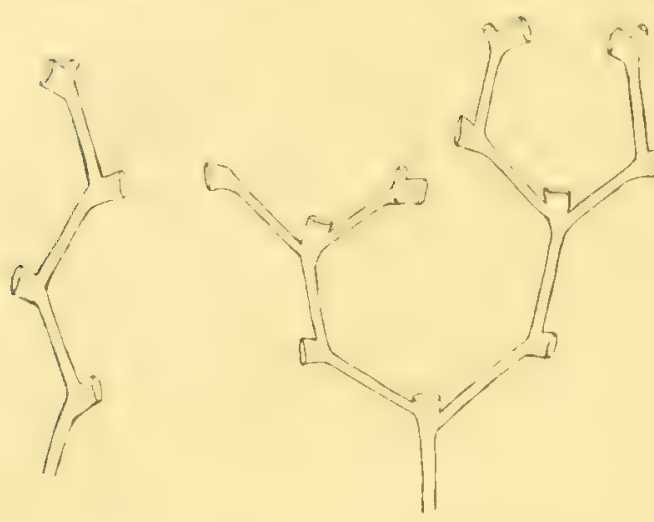

Fig. 13. Cladochoms michatini.

Kolonicn nacli den vorliegenden Fragmenten

reconstruirt: in walırer Grösse. schliessen, wie er in nebenstehender Textfigur 13 dargestellt ist. Die Grösse der Kolonien lässt sich selbstverständlich nicht bestimmen, ist aber für uns von untergeordneter Bedeutung. Da nur kleinere Bruchstücke vorliegen, lässt sich auch nicht entscheiden, ob die Kolonien von $\mathrm{Cl}$. michelini sich vorherischend in einer Ebene ausbreiteten, wie das für Pseudocladochonus typisch ist. Immer in einer Ebene erfolgte die Verzweigung nicht, denn es giebt Polypenkelche, aus denen drei Stïmmchen hervorgehen, welche nach drei verschiedenen Seiten gewendet sind (DE Kontxck und eigenes Material). Wenn aber ein Polypenkelch zwei Stämmchen abgiebt, gehen dieselben in einer Ebene nach entgegengesetzten Seiten ab. Die Polypenkelche scheinen auch meist in einer Ebene zu liegen, so auch bei dem in Textfig. i $2 \mathrm{~A}$ abgebildeten Fragmente.

Auch von anderen Cladochonus-Arten ist mir nichts sicheres darüber bekannt, ob die Kolonien sich in einer Ebene ausbreiteten. Doch weisen die kleinen Fragmente darauf hin. Alle Cladochonus-Arten scheinen diegleichen, in einer Ebene zikzakförmig gebogenen Stämmchen und abwechselnd zreihig angeordneten, nach entgegengesetzten Seiten gewendeten Polypenkelche besessen zu haben. Wenn dabei ein Polyp noch ein zweites Stämmchen abgab, lag dies, wenigstens bei Cl. michelini, in derselben Ebene, wie daraus hervorgeht, dass bei Polypen mit 2 Stämmchen, diese letztere nach entgegengesetzten Seiten abgehen (Textfig. I I und I 2); auch DE Konisck hebt dies hervor.

Nach diesen mehr allgemeinen Bemerkungen, möchte ich noch folgende Punkte besonders hervorheben, in wëlchen Pseudocladochonus mit Cladochonus übereinstimmt.

I" Bei beiden Genera gehen die Stämmchen niemals unmittelbar aus anderen Stämmchen, sondern immer nur aus den Polypenkelchen hervor, welche letztere dadurch die Stämmchen in kurze, aufeinander folgende Abschnitte gliedern.

$2^{0}$ Jeder Stammabschnitt endet in einen Polypen, der etwas nach einer Seite gebogen ist und einen nach der anderen Seite gerichteten neuen Stammabschnitt aus sich hervorgehen lässt.

3" Eine Verzweigung kommt dadurch zustande, dass ein Polyp zwei Stämmchen abgiebt, welche dann nach genau entgegengesetzten Seiten abgehen (von Clad. michelini sind auch Polypen bekannt, welche drei Stämmchen abgeben).

$4^{0}$ Durch schwache Stellen im sonst sehr festen Skelete zerbrechen die Kolonien von Pseudocladochonus in gleich grosse Fragmente, jedes bestehend aus einem längeren Stammabschnitte mit terminalem Polypen und einem aus letzterem hervorgehenden Basalstumpf eines neuen Stammabschnittes (Fig. IO, C, Taf. 3). Es zerfallen nun die Kolonien von Cladochonus michelini in genau solche Fragmente (Textfig. I I); grössere Fragmente sind selten, sodass sogar Minne Edwards und Halme $^{1}$ ) diese Art als eine isolirte Polypenform ohne Kolonienbildung 
betrachtet haben. Nach DE Koxrsck (1.c. p. I51) hat dies auch Fromextel getan in seiner "Introduction à l'étude des polypiers fossiles”, p. 348. Die Enden der Stämmchen können dabei sehr regelmässig abgebrochen sein (Textfig. I I, A, links). Dieses regelmässige Zerbrechen der Kolonien deutet darauf hin, dass an regelmässig angeordneten Stellen, welche den Biegungsstellen bei Psendocladochonus der Lage nach entsprechen, bei Clad. michelini schwache Querringe im Skelet auftreten. Die Möglichkeit, dass wir es hier auch bei letzterer Art mit den Stellen eines intercalaren Längenwachstums zu thun haben, ist nicht ohne weiteres von der Hand zu weisen.

Von anderen Cladochonus-Arten sind mir allerdings solche Biegungsstellen der Stämmchen nicht bekannt. Die Kolonien, welche ich gesehen habe (Cl. bacillarius M'Coy, Cl. campanulatus M'Coy und zwei andere, nicht bestimmte Arten) scheinen immer in unregelmässige Fragmente zu zerbrechen. Doch muss dies vielleicht darauf zurückgeführt werden, dass solche Bruchstellen (oder Biegungsstellen bei den lebenden Kolonien) zwar des Längenwachstums wegen zuerst überall vorhanden sind, dass aber mit dem Einstellen des Längenwachstums auch an diesen Stellen die normale feste Skeletbildung auftritt, sodass dieselben schwinden. Die etwas grösseren Fragmente von Clad. michelini, welche man bisweilen findet, weisen darauf hin, dass auch bei dieser Art diese Bruchstellen fehlen können, beziehungsweise nachträglich schwinden. Auch bei Pscudocladochomus findet man die gelblichen Querringe, an welchen die Biegungsstellen oder Bruchstellen der Kolonien kenntlich sind, bisweilen an den üblichen Stellen nicht. Am Fragmente von Clad. michelini, welches in Textfig. I $\mathrm{A}$ (S. 2 I) abgebildet ist, konnte ich keine schwachen Querringe im Skelete erkennen.

Einigermassen analoge Bruchstellen im Skelete sind mir unter allen Korallen nur von Alcyonaria bekannt, nämlich von den Achsen der Isisidae und Melitodidae. Bei den Hexacorallia, Tetracorallia und Rugosa scheint nichts ähnliches aufzutreten.

Neben diesen Punkten, in welchen Übereinstimmung zwischen Cladochonus und Pseudo. cladochonus besteht, finde ich jedoch auch einige, zum Teil nicht unwesentliche Unterschiede.

I. Erstens fehlt in den Stammabschnitten von Cladochonus das innere Skelet, welches bei Pseudocladochonus in den Scheidewänden zwischen den Stammkanälen entwickelt ist. Die Höhlung in den Stammabschnitten bei Cladochonus ist niemals in 8 Längskanäle geteilt. Einmal fand ich in meinem Materiale von Clad. michelini diesen sonst einheitlichen Raum durch ein Längsseptum in zwei Kanäle getrennt, doch mag hier eine abnormale Bildung vorgelegen haben. Allerdings beweist das Fehlen dieses inneren Skeletes nicht mit Gewissheit, dass der Raum in den Stammabschnitten auch beim lebenden Tiere ein einheitlicher Kanal war. Aber bei Pscudocladochoums bekomme ich doch den Eindruck, dass die Bedeutung der Scheidewände, ich möchte sagen der Grund ihres Daseins, darin gesucht werden muss, dass sie dem Skelete Gelegenheit bieten, Skeletsepten zu bilden, durch welche die Festigkeit der Stämmchen sehr erheblich erhöht wird. Bei Telesto und Coelogorgia, wo sie kein Skelet enthalten, sind die Scheidewände in den Stämmen doch immer schwach entwickelt.

2. Es soll hier auch auf die Tabulae in den Stammabschnitten von Cladochonus hingewiesen werden. Nichouson und Etmeridge ${ }^{1}$ ) geben an, dass sie Tabulae bei Cladochonus

1) Geological Magazine, New Serics, Decade 2, vol. 6, 1S79, p. 289. 
michclini und bei Aulopora gefunden haben. Leider ist, wie sie selbst hervorheben, die Bestimmung ihres Materiales von Cladochonus etwas zweifelhaft (es könnte nämlich auch Aulopora sein). Ich vermisse die Tabulae vollständig bei der Cladochomes species von Button Mould Ǩnob, von welcher Art ich mediane Längsschliffe durch drei verschiedene Stammabschnitte in einem Dünnschliffe durch ein Gesteinsfragment besitze. Bei Cladochonus michclini konnte ich die Anwesenheit von Tabulae nicht sicher feststellen bei einigen Stammfragmenten, welche ich zerbrochen habe, um danach zu suchen. Doch fand ich einige Male die Höhlung des Stammes abgeschlossen durch eine unregelmässige Skeletmasse, die möglicherweise als eine eigentümlich entwickelte Tabula gedeutet werden könnte; warscheinlich ist mir das aber nicht. Ich verfuge nur ïber einen medianen Längsschliff von Cladochomus michclini und derselbe zeigt keine Tabulae.

Von einer Kolonie von Aulopora repens (Mittel Devon, Villmar, Nassau) habe ich die Wandungen mit schwacher Salzsäure gelöst, sodass nur die Gesteinsmasse, welche die innere Höhlung ausfülte, erhalten blieb, wohl weil sie nicht lediglich aus Kalk bestand. Und diese Ausfiullungsmasse ist meist ununterbrochen und zeigt nirgends sichere Andeutungen von Tabulae. Die Monitoporidac besitzen keine Tabulae ${ }^{1}$ ).

Die Angabe von Nicholsox und Etheridge macht es sehr warscheinlich, dass bei den Auloporidac bisweilen Tabulae auftreten, aber eine Bestätigung namentlich des Auftretens derselben bei Cladochomus ist doch erwinscht, da meine Untersuchung zeigt, dass es keine constanten Bildungen sind. Bei Pscudocladochonus und allen anderen Genera der Telostidae sind niemals Tabulae gefunden worden. Hier liegt also, soweit wir jetzt urteilen können, ein Unterschied gegenüber den Auloporidac vor.

3. Die Verbindung der Magenhöhle eines Polypen mit den Kanälen eines, aus demselben hervorgewachsenen Stammabschnittes findet bei Pseudocladochonus mittels einiger ( 16 ) kleineren Öffnungen im Skelete statt. Dieselben entstehen dadurch, dass die Polypenwandung hier von einigen Solenia durchbohrt wird; ähnliche Solenia finden wir auch bei Telesto und anderen Telestidae (vergl. die Figuren 1 I und I2, Taf. 3). Bei den Auloporidae und den verwandten Moniloporidae finden wir statt der kleineren Öffnungen nur eine grössere. Schon die macroscopische Untersuchung von Aulopora repens und Cladochonus michelini lässt hierüber keinen Zweifel und dasselbe zeigen die Schliffe durch die Cladochonus species von Button Mould Knob. Die Ausfillungsmasse der Höhlungen von Polypen und Stolonen (Stammabschnitte) einer Kolonie von Aulopora repens (auspräpariert durch Lösung des Kalkskeletes in Salzsäure, wobei die Ausfullungsmasse zurückblieb) zeigt nur einen etwas geringeren Durchmesser am Übergang der Stolenen in die Polypenkelche, aber es besteht immer ein ausgedehnter Zusammenhang zwischen der Ausfullungsmasse der Stolonen und der Polypenkelche. Auch bei den Moniloporidae fehlt jede Andeutung von Solenia ${ }^{2}$ ); das Skelet lässt uberall eine geräumige Verbindung offen.

Dass bei den Auloporidac und Moniloporidac die Verbindung der Polypenhöhlungen

1) GRAB.t, proceedings Boston Soc. Nat. IIist., vol. $2 \mathrm{~S}, 1 \mathrm{~S} 99, \mathrm{p} .409,410,414$.

2) GRAbaU, l'roceedings Boston Soc. Xat. Hist., vol. $2 \mathrm{~S}, 1$ S99, p. 409, 424 ; pl. 2, fig. 1o (Schlift durch Ceratofora jacksoni), Bei Monilofera tiachiri und Coafopora disforta werden nit zunelmendem Alter die Höhlungen der Polypen von einander getrennt, indem das skelet die Offnung tiberdeckt, mittels welcher die jüngeren Polypen mit ilren ILtterpolypen in Verbindung stehen (GRAlit, 1. c. p. 4I 2, 4I5). 
mit der Höhlung der aus denselben hervorgehenden Stammabschnitte (Stolonen) niemals mittels einiger kleinen Löcher stattfindet, weist darauf hin, dass hier auch bei den lebenden Kolonien diese Verbindung mittels einer einzigen Öfnung stattfand und dass hier ein anderer Zustand vorhanden war, als wir bei den Telestidae finden, wo besondere Kanäle, die Solenia, diese Verbindung vermitteln.

4. Interessante Bildungen sind die septalen Längsleisten (septal striae, stries cloisonnaires) auf der Innenwand der Polypenkelche von Cladochomes. Mrlxe Edwarns und Harare, M'Cor und später DE KONINCK haben auf das Vorkommen derselben bei Cladochomus hingewiesen. DE KONInck und M'COy haben dieselben abgebildet und ich habe oben Copien ihrer Figuren gegeben (Textfig. 9 und 8, B, C, S. 20). An meinem Materiale von Clad. michelini fand ich dieselben nicht, aber Dr. Hinde zeigte sie mir an einer Cladochonus-Art von Keokuk, Iowa, in seiner Sammlung. Es kommen etwa 20 dieser sehr niedrigen Leisten in einem Polypenkelche vor.

Es wäre wichtig, wenn es sich ermitteln liess, dass diese Leisten ehemals in Mesenterien oder, wie die Sclerosepten der Hexacoralliae, regelmässig zwischen den Mesenterien gelegen haben müssen. Man würde dann für Cladochonus eine höhere Zahl der Mesenterien finden, als sie die Alcyonaria besitzen und es würde damit bewiesen sein, dass Cladochonus nicht zu den Alcyonaria gehört. Mir scheint es aber nicht möglich irgend einen Zusammenhang zwischen diesen Leisten, "septal striae”, und den Mesenterien nachzuweisen. Sie sind so niedrig, dass es zweifelhaft erscheint, ob sie ehemals in die Nagenhöhle der Polypen vorspringende Leisten bildeten.

Vielleicht lassen sie sich in Beziehung bringen zu den Stacheln (Trabeculae), welche bei Aulopora und Ceratopora im Inneren der Kelche auftreten können und welche bisweilen eine Anordnung in Längsreihen zeigen. Grabau hat diese regelmässige Anordnung von seiner Ceratopora dichotoma deutlich abgebildet ${ }^{1}$ ). Die Zahl der Langsreihen ist nach GraBAU bei Ceratopora I 6 bis 20, also etwa ebenso gross, wie die Zahl der Längsleisten bei Cladochonus michetini. Es können aber diese Stacheln auch unregelmässig zerstreut stehen, z.B. bei einer Aulopora (? A. tubaeformis Goldfuss) aus der Sammlung des Dr. Hinde; dies stimmt nicht iberein mit einer etwaigen Beziehung derselben zu Mesenterien. Dazu kommt, dass bei Monilopora die Stacheln oder Trabeculae, welche Grabau wohl mit Recht mit den Stacheln der Ceratopora homologisiert hat, sehr zahlreich sind und dicht gedrängt stehen, sodass für dieses Genus nicht angenommen werden kann, dass sie in den Mesenterien lagen. Aber dann fehlt auch fur die Stacheln von Ceratopora und einiger Aulopora-Arten jeder sichere Grund, dass sie in den Mesenterien lagen und wird dies für die Längsleisten oder "septal striae" auch ganz zweifelhaft.

Wir müssen also leider den Schluss ziehen, dass die Längsleisten und die Stachelreihen der Auloporidac und Moniloporidae uns nichts Sicheres lehren über die Zahl der Mesenterien bei den Polypen dieser Korallen. Eine Beziehung zu den Mesenterien erscheint in einigen Fällen möglich, aber mehr auch nicht. Es liegt hier wohl ein Unterschied zwischen den Auloporidae

I) Grabau, 1. c., Taf. 3, Fig. 14; vergl. auch ebenda S. 415. 
und den Tolestidae vor, welcher gegen eine engere Verwandtschaft beider Genera spricht, aber die genannten Bildungen der Auloporidae sind meines Erachtens nicht entscheidend für die Beantwortung der Frage nach der Zugehörigkeit der Auloporidae zu den Alcyonaria im verneinenden Sinne.

SII. Die mikroskopische Structur des Skeletes bei Cladochonus.

Pscudocladochonus besitzt, wie S. 6 ausführlicher beschrieben wurde, ein Skelet, welches aus Kalkspiculae aufgebaut ist. Dieselben verschmelzen grösstenteils zu einer festen Skeletmembran, in welcher kleinere Löcher den ehemals grösseren Lücken zwischen den Kalkkörperchen entsprechen. Die Grenzen der Scleriten sind zum Teil verwischt, aber an manchen Stellen doch noch sehr deutlich zu erkennen. (vergl. Textfig. 4-6, S. 7).

Über die mikroskopische Structur des. Skeletes bei Cladochonus und Aulopora liegen keine Angaben vor, welche auf der Untersuchung sehr diinner Schliffpräparate beruhen. Aus der Litteratur geht nicht hervor, ob das Skelet dieser Genera, wie bei Psendocladochonus, aus verschmolzenen Scleriten aufgebaut ist. Ich habe versucht, diesen letzteren Punkt durch eigene Untersuchung aufzuklären, mehr jedoch habe ich bei meiner Untersuchung nicht erstrebt. Es war nicht meine Absicht, der mikroskopischen Skeletstructur der Auloporidae eine eingehende Untersuchung zu widmen.

Ich verfügte für meine Untersuchung über einige Schliffe durch verkieseltes Material von Cladochonus michelini, über einen Schliff durch ein Kalksteinfragment mit zahlreichen Stamm-

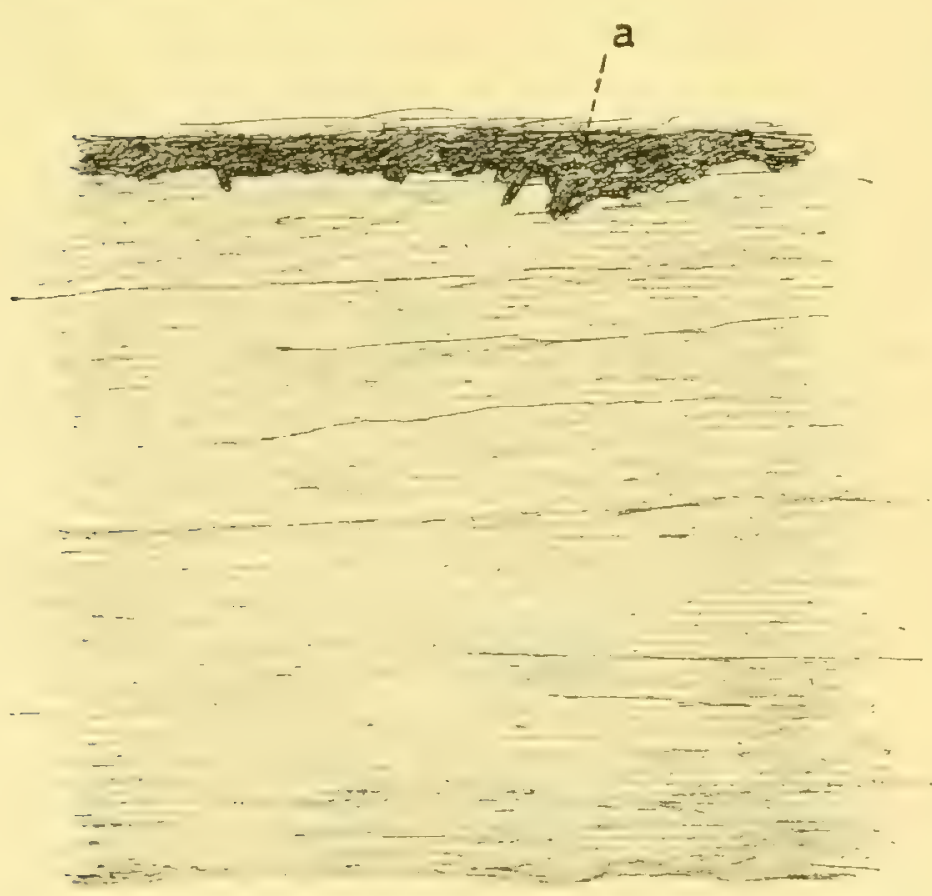

Iig. I4. Chadechonus species; medialer Längsschlift durch die Wandung eines Stämmchens; a dunkle Ausfüllungsmasse des Stamıkanales. X 300.

mehrere Fragmente einer Cladochonus-Art längs abschnitten einer Cladochonus-Art von Button Mould Knob, Kentucky (nicht verkieselt) und über einen Längsschliff durch einen Stammabschnitt (Stolo) von Aulopora repens (ebenfalls nicht verkieselt). Diese Schliffe wurden alle nach meinen Angaben vom Rheinischen Mineralien Contor, Dr. F. KRANTz in Bonn, angefertigt und erlauben auch die Untersuchung mittelst sehrr starker Vergrösserungen (Öl-Immersion 1/12). Wiewohl diese Schliffe für ein vergleichendes, detaillirtes Studium des Skeletes der Auloporidae nicht ausreichen (es sind deren zu wenige), erlauben sie uns doch einige Schlüsse bezuiglich des Aufbaues dieses Skeletes zu ziehen.

Sehr deutliche Bilder giebt der Dünnschliff durch das Gesteinsfragment von Button Mould Knob, Kentucky, in welchem Schliffe und schräg, nur vereinzelte mehr quer, 
geschnitten worden sind. Dieses Präparat zeigt, dass das Skelet aus sehr feinen, der Längsachse des Stammabschnittes parallelen Säulen aufgebaut ist (Textfig. I. ); dieselben sind auch auf tangentialen Längsschliffen deutlich, während die Stellen, wo die Wandung mehr quer geschnitten worden ist, beweisen, dass die Längsstrichelung nicht auf concentrische Schichtung des Kalkskeletes zurückgeführt werden kann.

Dasselbe Bild zeigt auch ein medialer Längsschliff durch das Stammskelet von Aulopora repens.

Etwas weniger deutlich und regelmässig ist der Säulenbau des Skeletes verkieselter Exemplare von Cladochomes michclini, sowohl bei medialem als tangentialem Längsschliffe. Man vergleiche hierzu Textfig. I 5 . Bei sehr starker Vergrösserung (C. ZEIss, Öl-Immersion

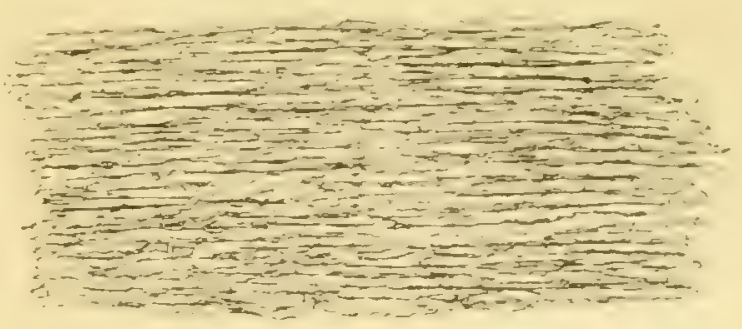

Fig. 15. Cladochonus michclini; medialer Längsschliff durch die Wandung eines Stämmchens. X 300. $1 / 12$ und Compensations-Ocular 8) sieht man, dass die Säulchen vielfach zerbrochen sind (Textfig. I6), sodass es stellenweise aussieht, als ob die Säulchen aus aneinander gereihten kleineren Kalkkörperchen beständen. Es giebt sogar Stellen in meinen Präparaten, wo das Skelet aus isolirten, parallel gelagerten, nadelförmigen Scleriten zu bestehen scheint, aber andere Stellen derselben Präparate zeigen immer deutlich die Säulenstructur. Veränderungen bei der Fossilisation dürften bei diesen Präparaten die geringere Deutlichkeit der Säulenstructur verursacht haben; das Material ist verkieselt, was bei

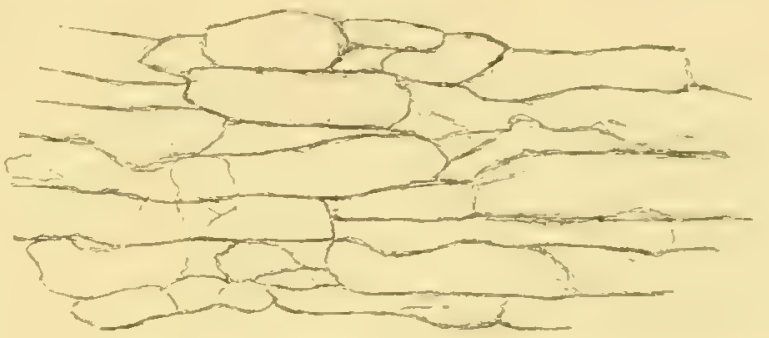

Fig. 16. Cludochonus michelini; medialer Längsschliff durch die WVandung eines Stammabschnittes. $\times 933$. den deutlich aus Säulen aufgebauten Skeleten von Aulopora und von der Cladochomus-Art von Button Nould Knob nicht der Fall ist.

Die Dünnschliffe zeigen also einen ganz anderen Bau des Skeletes als übereinstimmen wiirde mit einem Aufbau desselben aus isolirt auftretenden, später verschmolzenen, Kalkkörperchen, wie wir es bei Psezdocladochonus gefunden haben.

Selbst wenn wir die Kalkkörperchen-ähnlichen Abschnitte, in welche bei Cladochonus michelini die Skeletsäulen geteilt sind (Textfig. 15, 16), als Scleriten deuteten (welche Deutung ich aber zurückweisen muss), wirde die Ähnlichkeit mit dem Skelete des Psendocladochonus noch keine vollständige sein. Es würde dann bei Cladochonzs ein aus zahlreichen, dicht über einander geschichteten Scleriten zusammengesetztes Skelet vorliegen, dessen Dickenwachstum durch Zufügung neuer Scleriten an der Innenseite des schon vorhandenen Skeletes stattfinden miisste. Dagegen geschieht, wie oben S. 15 beschrieben wurde, das Dickenwachstum des Skeletes bei Pseudocladochonus durch Wachstum der grossen, in einer Schicht liegenden Scleriten, nicht durch Bildung neuer Kalkkörperchen.

Auch der geschichtete Bau des Skeletes bei den Moniloporidac und der feingeschichtete Bau desselben bei Syringopora ${ }^{2}$ ) sprechen gegen einen Aufbau des Skeletes der nahe verwandten Auloporidac aus mesodermalen Kalkkörperchen. Werssersuc erwähnt für Syringopora auch eine 
faserige Structur, welche der Säulenstructur des Skeletes der Auloporidae nahe kommen dürfte.

Weil nun aber die Skeletstruktur von Cladochonus und Psendocladochonus nicht auf einen ähnlichen Bau des Skeletes hinweist, kann ich keine enge Verwandtschaft zwischen diesen beiden Genera annehmen.

S I2. Ergebnisse der Vergleichung von Pseudociadochonus mit fossilen Formen und seine Verwandtschaft mit Cladochonus.

Wenn wir nach der Erörterung des Baues der Auloporidae in den Şऽ ro und I I mit Zusammenfassung des Thatsachenmateriales, uns eine Meinung über die Verwandtschaft von Pseudocladochomus mit Cladochonus bilden wollen, so sehen wir, dass einerseits eine sehr grosse Ähnlichkeit im Habitus fur eine enge Verwandtschaft spricht, dass aber andrerseits doch wesentliche Unterschiede im Baue beider Genera bestehen, welche gegen einer Verwandischaft sprechen. So zeigt Cladochonus in seinem Skelete am Abgang der Stammabschnitte seitwärts aus den Polypenkelchen nur eine grosse Öffnung, sodass von einer Verbindung der Polypenhöhlungen mit einander mittels Solenia (engerer Entoderm-Kanäle) in einer Art, wie sie für die Telestidae typisch ist, nichts angedeutet ist. Eine Vergleichung von Cladochonus mit Aulopora und Syringopora macht es warscheinlich, dass die Stammabschnitte des ersteren aus kriechenden Stolonen hervorgegangen sind, und nicht die basalen, umgebildeten Abschnitte der Polypen selbst sind, wie das bei Psendocladochonus der Fall ist; namentlich spricht hierfuir, dass bei Aulopora ein Polyp den verschmolzenen Enden von zwei oder drei der kriechenden Stammabschnitte terminal aufsitzen kann; denn eine Verschmelzung der Stolonen ist bei den Korallen (Alcyonaria und Zoanthidae) ziemlich verbreitet, während mir von einer Verschmelzung der terminalen Abschnitte von zwei bis drei Polypen zu einem einzigen Polypenkelche kein Beispiel bekannt ist.

In den Längsleisten (stries cloisonnaires oder septal striae) im Inneren der Polypenkelche, sowie in den Stacheln (Trabeculae) und den Tabulae liegen Bildungen der Auloporidae vor, welche zwar meines Erachtens nur mit grösster Reserve für Schlüsse auf den Bau der Weichteile verwendet werden können, die aber doch jedenfalls von den Telestidae nicht bekannt sind.

Auch der feinere Aufbau des Skeletes der Auloporidac erweist sich als verschieden von dem, was wir bei Pseudocladochonus finden. Ginge es hier lediglich um die Prifung einer möglichen nahen Verwandtschaft von Pseudocladochonus mit Cladochonzs, so könnte man dieselbe noch zu retten versuchen, indem man eine Änderung im Modus der Skeletbildung bei Cladochonus annähme. Der Übergang des spiculären Pseudocladochonus-Skeletes in ein aus Säulen aufgebautes bei Cladochonus wäre denkbar. Aber Aulopora, welches Genus, wenn man an eine Verwandtschaft mit Pseudocladochonus denkt, doch entschieden primitiver erscheint als letzteres Genus, hat auch schon ein nicht-spiculäres Skelet. Cladochonus stammt wohl höchst warscheinlich von Aulopora ab und zeigt denselben Skeletbau wie letzteres Genus. Eine Verwandtschaft des Pseudocladochonus mit den Auloporidae erscheint nur möglich, wenn dieses Genus denselben Aufbau des Skeletes besässe, wie letztere Familie, und das ist nicht der Fall. Auch die Annahme, es wäre ein in feinen Säulen abgesondertes oder ein geschichtetes Skelet der primitivere Typus, 
das spiculäre Skelet von Pseudocladochonus der abgeleitete Zustand ${ }^{1}$ ), ist hier nicht haltbar wegen der Verwandtschaft von Pseudocladochomes mit den Telestidae, wodurch eine Abstammung des Pseudocladochonus von primitiven Alcyonaria (Stolonifera Hickson) mit spiculärem Skelete nicht zweifelhaft ist.

So bleibt uns nur übrig, die äussere Ähnlichkeit von Cladochomıs mit Psezdocladochonus auf Convergenz zurück zu führen. Eine der grossen äusseren Ähnlichkeit entsprechende sehr enge Verwandtschaft kann hier wegen des verschiedenen Baues des Skeletes und anderer Unterschiede nicht angenommen werden. Dieser Ansicht über die Verwandtschaft beider Genera habe ich im Namen, den ich für die neue Siboga-Art gewählt habe, Ausdruck gegeben, indem ich dieselbe Psezdocladochonus genannt habe.

\section{Wichtigste Ergebnisse.}

In Psendocladochonus hicksoni liegt ein neuer, zu den Telestidae gehöriger Alcyonarior vor. Derselbe zeichnet sich vor allen andern Telestiden dadurch aus, dass bei ihm keine Differenzirung in lange stamm- resp. astbildende axiale Polypen und kurze Seitenpolypen statt gefunden hat. Alle Polypen sind hier vom gleichen Baue, alle gesondert in einen distalen Kelchabschnitt, den Polypen in engerem Sinne, und einen längeren basalen Stammabschnitt. In den Stamm. abschnitten findet eine Verschmelzung der Innentänder der Mesenterien statt, wodurch die Magenhöhle der Polypen dort in S Längskanäle gesondert wird.

Abgesehen von dem aus isolirten Scleriten bestehenden Skelete der retractilen Abschnitte der Polypen, besteht das Skelet aus verschmolzenen Scleriten; dieselben liegen in einer Schicht und verschmelzen zu einer dicken Skeletmembran. Dieses Skelet wird auch in den Scheidewänden der Stammkanäle gebildet und die acht radial geordneten Skeletlamellen treffen in der Achse der Stammabschnitte zu einer Skeletsäule zusammen. Mit diesem Baue der Stammabschnitte bildet Psendocladochonus einen neuen Bautypus des Stammes einer Kolonie von Alcronaria. Derselbe ist aber vor Allem interessant, weil er einen von STUder bei seiner Ableitung der Gorgoniden (Axifera) von den Telestiden schon postulirten Bautypus vorführt. Ob diese Ableitung sich auch jetzt, mit dieser neuen Stüze, genügend begründen lässt, dariiber habe ich S. 17 einige Bemerkungen aufgenommen; mir scheint das recht fraglich.

Das feste Skelet in den Scheidewänden der Stammabschnitte setzt sich nach oben zu continuirlich fort in die vier dorsalen Mesenterien der Polypen (im engeren Sinne). Es ist dies das erste Mal, dass ein festes Skelet in den Mesenterien eines Alcyonariers gefunden worden ist.

Pscudocladochoms zeigt eine sehr weitgehende Ähnlichkeit mit den fossilen Auloporidac, besonders mit dem Genus Cladochonus M'Coy; dieselbe beruht aber auf Convergenz und ist kein Zeichen einer engeren Verwandtschaft.

I) Eine solche Ablcitung des spiculïren Skeletes aus dem Skelete der 'Tabulaten hat SAkDesox befurworict (Neues Jahrluch fir Mineralogie, Geologic und Palaeontologie, bd ro, leilage Band, rS96, p. 249-476); WETsERMrL (Zeitschr. der I)eutschen (ieolog. Gescllschaft, 13d. 50, 1898 , p. 54-78) hat aber dieser Ableitung gewichtige (iründe cntgegengelmacht und auch ich kann nich det SARDESOX'schen Ansicht nicht anschliessen. 


\section{Nachweis der Litteratur.}

Bourne, Gilberl C. The Anthozoa; A Treatise on Zoology, edited by E. RAY LANKEsTer, Prt 2, The Porifera and Coelentera, London 1900.

Cimaman, F. On some fossils of Wenlock Age from Mulde, near Klinteberg, Gotland; Annals and Magazine of Natural History, ser. 9, vol. 7, 1901, p. I4I-I60.

DANA, J. D. Zoophytes; United States Exploring Expedition during the years I838, 3839,1840 , I 84 I, 1842 , under the command of CHARLES WILKES, U.S. N.; Philadelphia, I 848.

DELAgE, Yyes et Hérouard, Edgard. Traité de Zoologie Concrète, Tome 2, 2 ième Partie, Les Coelentérés, Paris, Igor.

EDWARd, Milje et Haime, Jules. Monographie des Polypiers Fossiles des Terrains Palaeozoiques; Archives du Muséum d'Histoire Naturelle, 'Tome 5, $185 \mathrm{I}$.

GRABAU, A. IV. Moniloporidae, a new Family of Palaeozoic Corals; Proceedings of the Boston Society of Natural History, vol. 28, N. I6, I 899, p. 409-424.

HaEckel, ERNST. Systematische Phylogenie. Berlin, I896.

Hickson, SrDNEy J. The Structure and Relations of Tubipora. Quarterly Journal of Microscopical Science, New Ser., vol. 23, I $88_{3}$, p. $556-577$.

_- Preliminary Notes on certain Zoological Observations made at Talisee Island, North Celebes; Proceedings Royal Society London, vol. 40, I886, p. 322-325.

- Alcyonaria Stolonifera; Transactions of tlie Zoological Society of London, vol. I 3, Prt 9, I894, p. 325-347.

_- The Anatomy of Alcyonium digitatum; Quarterly Journal of Microscopical Science, new ser., vol. 37 , I 895 , p. $343-385$.

_ - and Hiles, ISA L. The Stolonifera and Alcyonacea collected by Dr. WILLEY in New Britain, etc.; A. Willey's Zoological Results, Prt 4, Cambridge, I900, p. 493-508.

- - The Alcyonaria of the Maldives, Prt I; Fauna and Geography of the Maldive and Laccadive Archipelagoes, vol. 2, Prt I, I903, p. $473-502$.

- Coelentera; the Cambridge Natural History, edited by S. F. HARMer and A. E. SHIPLEY, vol. I, Protozoa, Porifera, Coelenterata, Ctenophora, Echinodermata, London, 1906.

Kocii, G. von. Anatomie der Orgelkoralle, Jena, I\$74.

_- Bemerkungen über das Skelet der Korallen; Morphologisches Jahrbuch, Bd 5, r879, p. 316-323.

_- Anatomie der Clavularia prolifera n. sp.; Morphologisches Jahrbuch, Bd 7, I882, p. 467-487.

_- Vorläufige Mittheilungen über die Gorgonien (Alcyonaria Axifera) von Neapel und über die Entwicklung der Gorgonia verrucosa; Mittheilungen aus der Zoologischen Station zu Neapel, Bd 3 , 1882 , p. $537-550$.

_- Die Gorgoniden; Fauna und Filora des Golfes von Neapel, Berlin, 1887 .

KüLlKKer, A. Vox. Bciträge zur Kenntniss der Polypen (Pseudogorgia Godeffroyi); Verhandlungen der physikalisch-medicinischen Gesellschaft in Wüzburg, neue Folge, Bd 2, IS72, p. II-2S. 
KOnINCK, M. L. G. DE. Nouvelles recherches sur les animaux fossiles du Terrain Carbonifére de la Bel. gique: Mémoires de l'Académie Royale des Sciences, des Lettres et des Beaux-Arts de Belgique, Tome $39,1872$.

KÜKEnTHaL, W. Alcyonacea; Wissenschaftliche Lìrgebnisse der deutschen Tiefsee-Expedition auf dem Dampfer Valdivia, Bd 13, 1906, p. I-III.

IIAC COy, F. On the Fossil Botany and Zoology of the Rocks associated with the Coal of Australia; the Annals and Magazine of Natural History, vol. 20, 1847, p. 227.

MAY, WaLther. Beiträge zur Systematik und Chorologie der Alcyonaceen; Jcnaische Zeitschrift fur Naturwissenschaft, Bd 33, neue Folge, Bd 26, 1900, p. I-ISo.

MENnEking, Friedrich. Über die Anordnung der Schuppen und das Kanalsystem bei Stachyodes ambigua (Stud.), Caligorgia flabellum (Ehrbg.), Calyptrophora Agassizii (Stud.), Amphilaphis abietina (Stud.) und Thouarella variabilis (Stud.); Archiv für Naturgeschichte, Jahrg. 7 I, Bd I, 1905, p. 245-266.

MÜllek, F. Über Balanus armatus etc.; Archiv für Naturgeschichte, Jahrg. 33, Bd I, I867, p. 330-332.

Nicholson, H. A. On the Structure and Affinities of the Tabulate Corals of the Palaeozoic Period; London, 1879.

- and Etheridge, R. On the microscopic structure of Three Species of the Genus Cladochonus; The Geological Magazine, new Series, Decade 2, vol. 6, is79, p. 289-296.

Sardeson, F. W. Über die Beziehungen der fossilen Tabulaten zu den Alcyonarien; Neues Jahrbuch für Mineralogie, Geologie und Palaeontologie, IO. Beilage Band, I896, p. 249-476.

Studer, Th. Versuch eines Systemes der Alcyonaria; Archiv für Naturgeschichte, Jahrg. 53, I3d I, 1887 , p. $1-74$.

Alcyonaires provenant des Campagnes de l'Hirondelle (I886-1888); Résultats des Campagnes Scientifiques accomplies sur son Yacht par Albert rer Prince Souverain de Monaco, Fascicule 20, 190 r.

Versluys, J. Primnoidae; Die Gorgoniden der Siboga-Expedition II; Uitkomsten op zoologisch, botanisch, oceanographisch en geologisch gebied, verzameld in Nederlandsch Oost-Indië I899-1900, Monugraphie XIII $a$, Leiden, 1906.

Weissermel, W. Sind die Tabulaten die Vorläufer der Alcyonarien?; Zeitschrift der Deutschen geologischen Gesellschaft, Bd 50, I898, p. 54-78.

Wright, E. P. and Studer, Th. Report on the Alcyonaria; Report on the scientific Results of the Voyage of H. M.S. Challenger, etc.; Zoology, vol. 31, 1889.

Zittel, Karl A. von. Handbuch der Palneontologie, Bd I, Protozoa, Coelenterata, Echinodermata, Molluscoiden; München und Leipzig, I876-I880.

- Grundzuige der Palaeontologie; München und Leipzig, I895. 


\section{Inhaltsverzeichnis.}

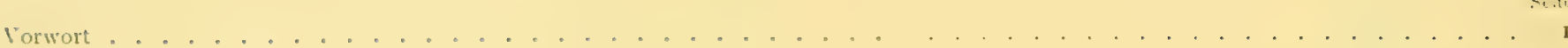

Pscudacladochonus hicksoni nov. gen. nov. species; Fundstellen und Material. .................... . . . . .

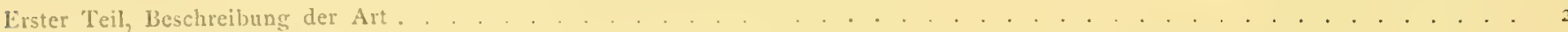

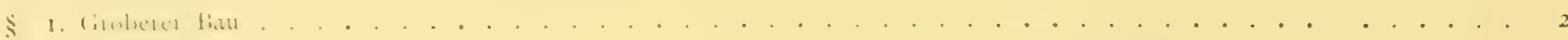

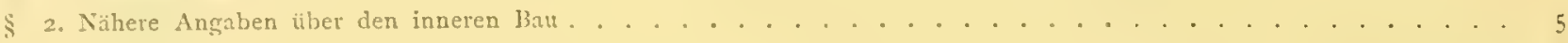

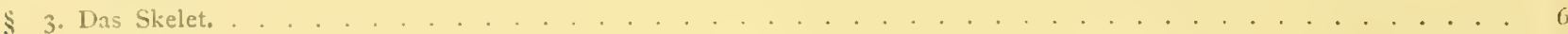

Zweiter Teil, die Verwandtschaft von Psendocladochonus mit lebenden Alcyonaria. . . . . . . . . . . . . . . . . . . I I

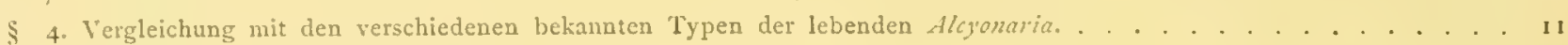

$\$$ 5. Vergleichung von Psendocladochonus mit den Telestiduc. . . . . . . . . . . . . . . . . . . . . . . . . I 3

$\$$ 6. Diagnose des neuen Genus Pscritochdochonus . . . . . . . . . . . . . . . . . . . . . . . I5

\$ 7. Beziehungen der Telestidae zu den Gorgonidin. . . . . . . . . . . . . . . . . . . . . . . . . . . I6

Dritter Teil, die Verwandtschaft von Psidochadochonus mit dem fossilen Genus Cladochonus M'Coy. . . . . . . . . . . . IS

s S. Einleitende Bemerkungen; Material. . . . . . . . . . . . . . . . . . . . . . . . . IS

\$ 9. Die wichtigsten Beschreibungen und Diagnosen des Genus Cladochonus M'Coy. . . . . . . . . . . . . . 20

\$ ro. Vergleichung des Banes von Pstudochadochonus und Cladochomus . . . . . . . . . . . . . . . . . . 2 I

$\$$ II. Die mikroskopische Structur des Skeletes bei Cladochonns . . . . . . . . . . . . . . . . . . . . . . 26

12. Ergebnisse der Vergleichung von Psiudocladochonus mit fossilen Formen und seine Verwandtschaft mit Cladochonus. 2S

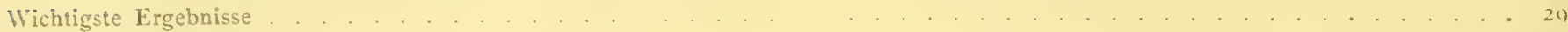

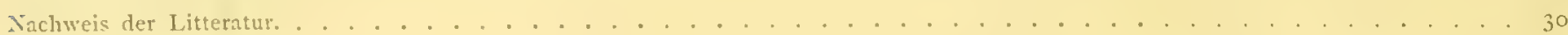


TAFELN 


\section{TAFEL II.}

Alle Figuren sind Abbildungen von Querschnitten durch einen Polypen von Pseudocladochonus; Vergrösserung $\times$ I40. Fig. I ist nach einem Schnitte aus einer andern Serie als Fig. 2-7 angefertigt. Die Reihenfolge der Schnitte ist von der Mundseite ab nach der Polypenbasis zu.

Die Figuren sind mit dem Prisma gezeichnet, aber etwas vereinfacht; die Lücken im Mesenchym (Mes, in Fig. I und 2) waren vom Skelete ausgefullt; durch das Entkalken ist der Umriss der Sclnitte etwas unregelmässig geworden.

Für alle Figuren gültige Bezeichnungen: Ent. Entoderm; ect. Fil. ectodermales Gastralfilament; int. Fil. entodermales Gastralfilament; Mes. in Fig. I und 2 Mesenchym, in Fig. 5 und 6 Mesenterium; Mes. doirs. dorsales Mesenterium; Musk. Muskelfahne; Si. Siphonoglyphe; St. Kan. Stammkanal; Stom. Stomodaeum.

Fig. 1. Querschnitt durch den tieferen Teil des Stomodaeum.

Fig. 2, 3, 4, 5. Querschnitte durch einen Polypen und den daraus hervorwachsenden Stammabschnitt.

Fig. 6 und 7. Querschnitte durch die Polypenbasis unterhalb des seitwärts abgehenden Stammabschnittes; Fig. 7 fällt unterhalb der Gastralfilamente. 


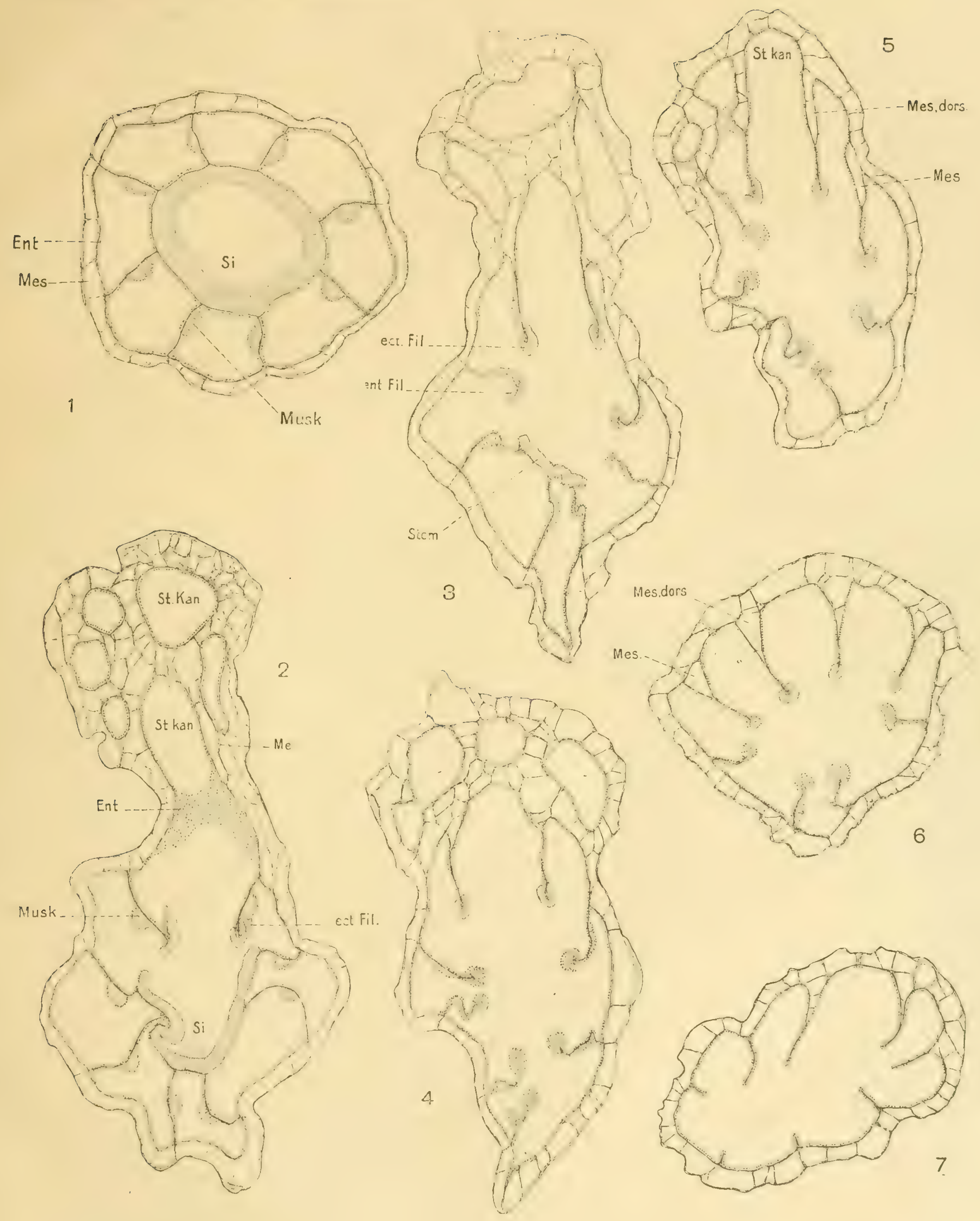

J. Verstuys del. 

$=$ 


\section{TAFEL III.}

Fin. 8 und 9. Fortsetzung der auf Tafel II gegebenen Serie von Abbildungen von Querschnitten durch einen Polypen. Vergrösserung $\times \mathbf{I} 40$.

Fis. S. Der Schnitt geht durch den Übergang des Polypen in den Stammabschnitt; 4 Mesenterien sind mit einander verschmolzen, 4 haben noch einen freien Innenrand.

Fig. 9. Querschnitt durch den oberen Teil des Stammabschnittes; man sieht die acht von Entoderm ausgekleideten Stammkanäle.

Iig. I0. Fragmente von Psendocladochonus hicksoni; A und C von Stat. I66, B von Stat. I44. Das in Fig. A abgebildete Fragment ist von einer Spongie überwachsen, welche aber nicht mit dargestellt ist (vergl. Textfig. I C, S. 2). Bei B ist ein besonders langer Stammabschnitt abgebildet. p. Polypenkelche; s. Stamm; z Seitenzweige; die schwachen Querringe im Skelete (Biegungsstellen), sind durch Punktirung angedeutet.

Fig. Ix. Schematische Darstellung des Baues von Pseudocladochonus; vergrössert. A ein der Länge nach und in der Verzweigungsebene der Kolonie gespaltenes Fragment; B Polyp und basaler Stammabschnitt, senkrecht zur Verzweigungsebene der Kolonie der Länge nach gespalten um die basalen Mündungen der Längskanäle eines Stammabschnittes in die Magenhöhle des Polypen zu zeigen (bei S.). Bezeichnungen : ect. Fil. ectodermales Gastralfilament; $K$. Polypenkelch mit darin zurückgezogenem distalem Polypenabschnitt; S. Solenia, die Öffnungen der Stammkanäle an ihrem unteren Ende in die Magenhöhlen der Polypen; Sch. Scheidewände zwischen den Stammkanälen; St. Stammabschnitte; St. Kan. Stammkanäle; Stom. Stomodaeum.

1.is. 12. Schematische Darstellung des Baues von Telesto; vergrössert. Die Figur stellt ein der Länge nach gespaltenes Fragment dar. Bezeichnungen wie in Fig. I I dazu: ent. Fil. die Mesenterien, welche ganz oben entodermale Gastralfilamente tragen; m.s. MIundscheibe des Polypen; T. Tentakel.

Ii1. 13. Psendocladochonns, Stamm quer; das Skelet ist dunkel schraffirt; $\times$ I22.

Iig. I4. Pseudocladochonus, Basis einer auf einer Muschelschale festgewachsenen Kolonie (Textfig. I D, S. 2); $s$. Stolonenartige Auswiuchse der Stammbasis; $p$. Polypenkelch; $\times$ I 8 . 
Sibga-Expcditic XIIlc. J. Versuurs. P'seudocladochonus.

III.
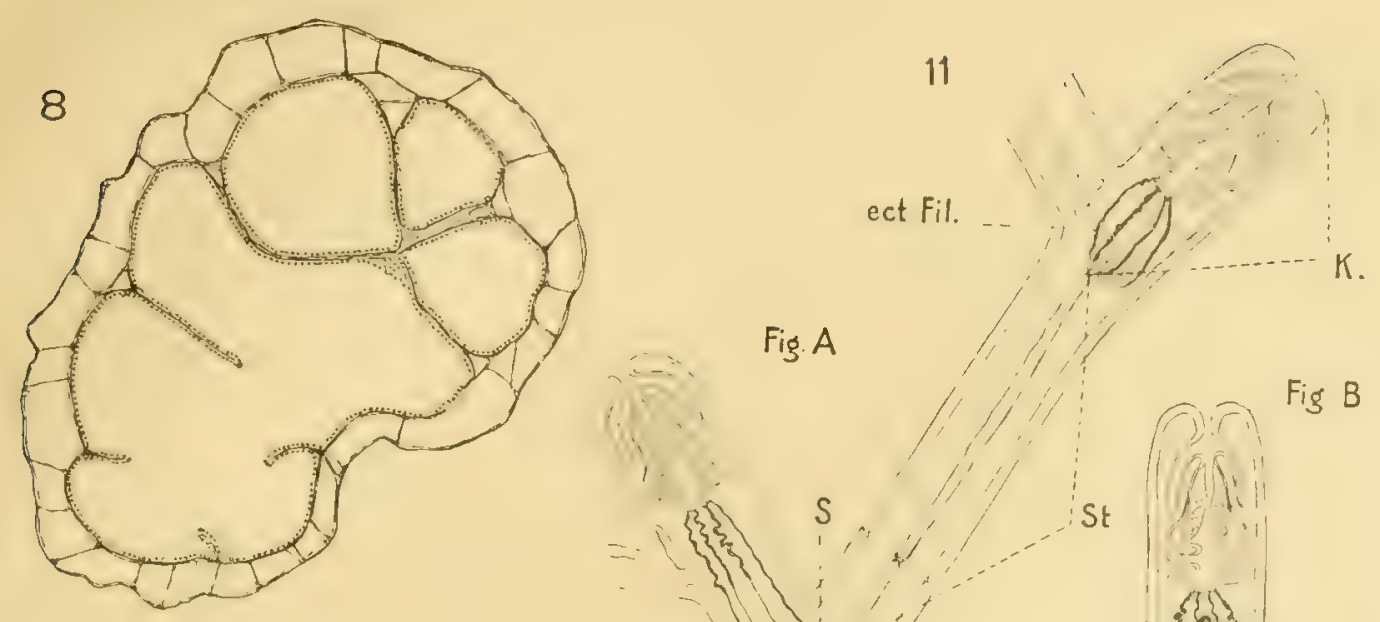

Fig. A
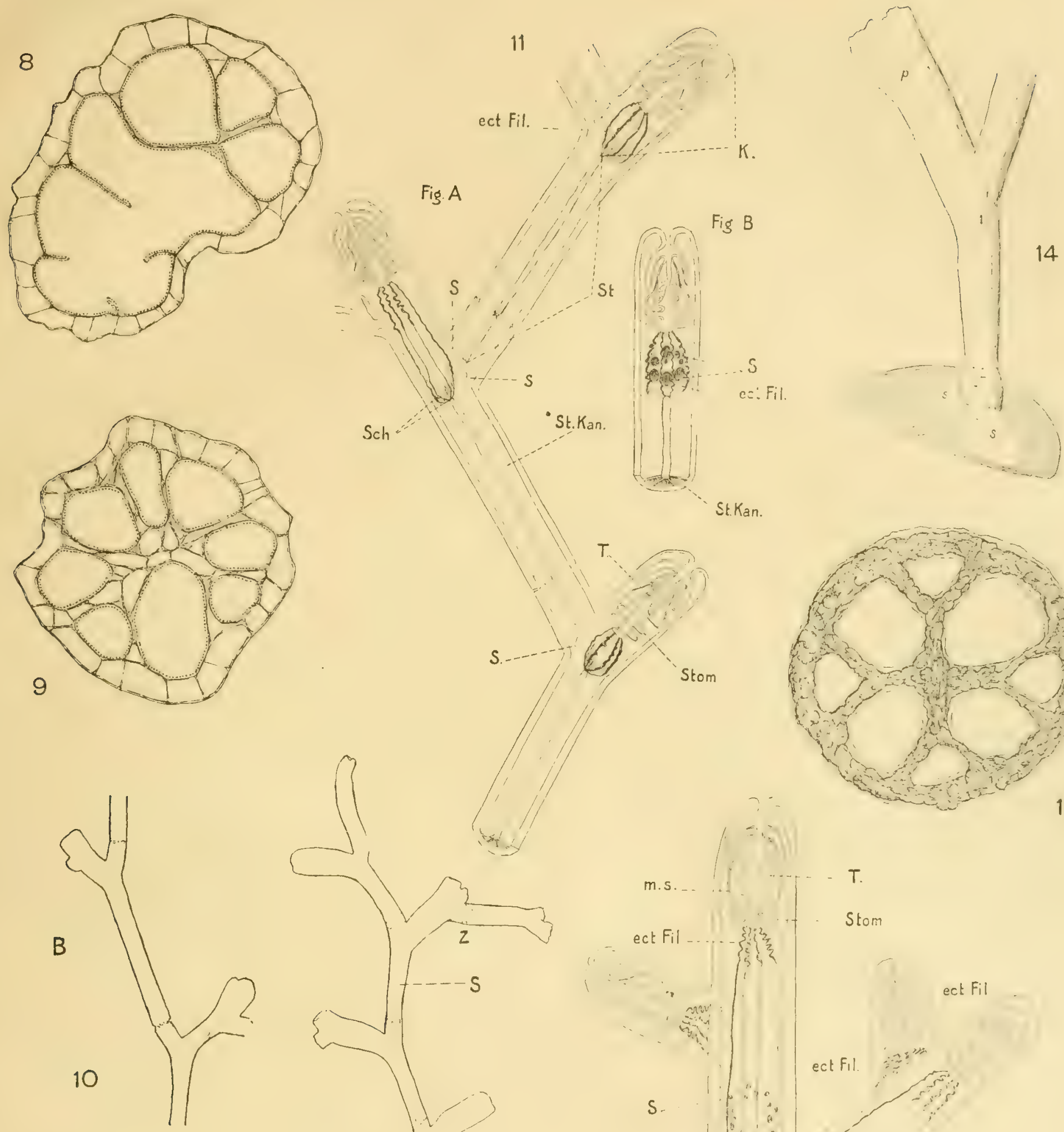

$$
\text { StKan. }
$$
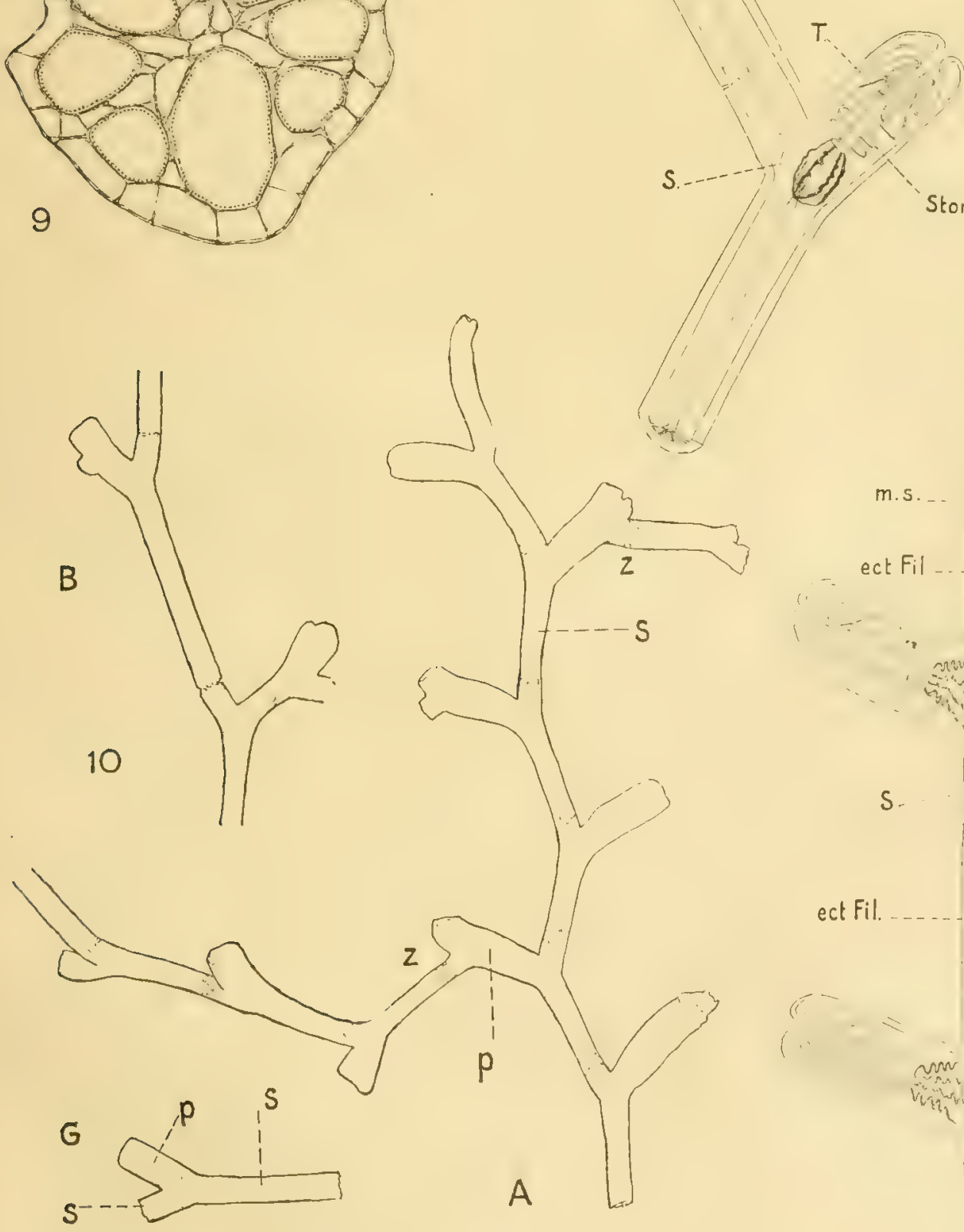

J. Verslurs del.

A
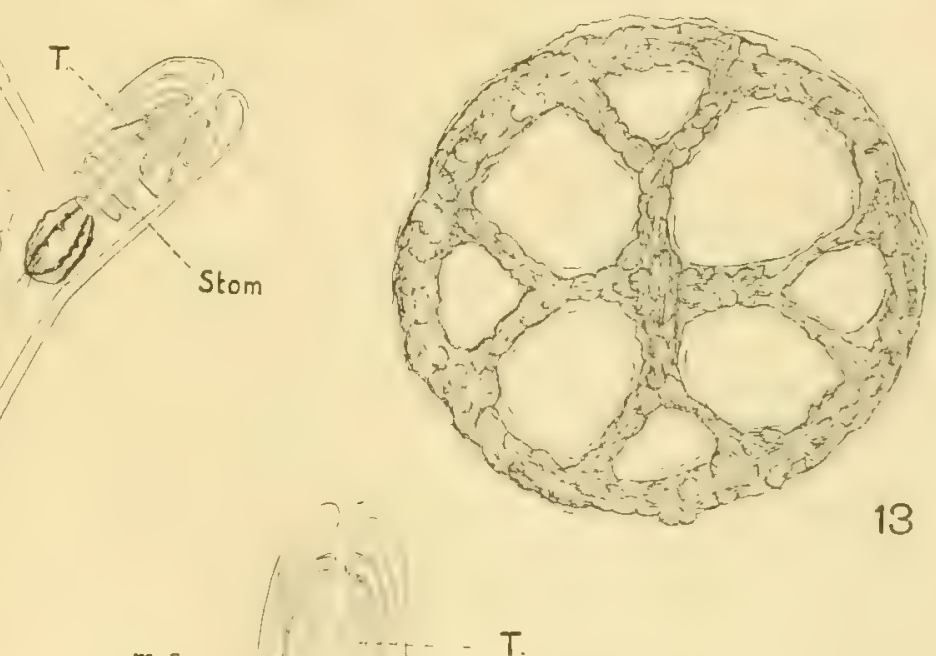

13

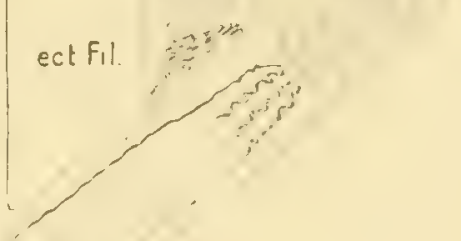





\section{CONDITIONS GÉNÉRALES DE VENTE.}

$\Upsilon^{3}$. L'ouvrage du "Siboga" se composera d'une série de monographies.

$2^{\circ}$. Ces monographies paraîtront au fur et à mesure qu'elles seront prêtes.

$3^{\circ}$. Le prix de chaque monographie sera différent, mais nous avons adopté comme base générale du prix de vente: pour une feuille d'impression sans fig. flor. 0.I 5 ; pour une feuille avec fig. flor. 0.20 à 0.25 ; pour une planche noire flor. 0.25 ; pour une planche coloriée flor. 0.40 ; pour une photogravure flor. 0.60 .

$4^{\circ}$. Il y aura deux modes de souscription:

a. La souscription à l'ouvrage complet.

b. La souscription à des monographies séparées en nombre restreint.

Danș ce dernier cas, le prix des monographies sera majoré de $25 \%$.

$5^{\circ}$. L'ouvrage sera réuni en volumes avec titres et index. Les souscripteurs à l'ouvrage complet recevront ces titres et index, au fur et à mesure que chaque volume sera complet. 


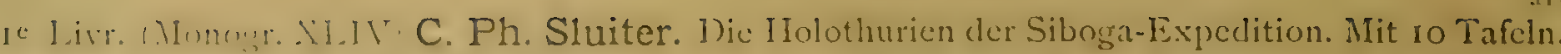

2: Livr. (Monogr. LX) E.S. Barton. The genus. Halimeda. With 4 plates.

$1.80,22.40$

3. Lin. Mfon: 1. I Max Weber. Introluction et description de l'expédition. Avec Liste des

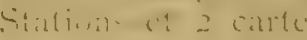

4. Liv. IJm, I. II, G. F. Tydeman. Description of the ship and appliances used for scientific cxploration. With 3 plates and illustrations.

$=$ L i.r. Munour. XI.VII) H. F. Nierstrasz. The Solenogastres of the Siboga-Exp. With 6 plates.

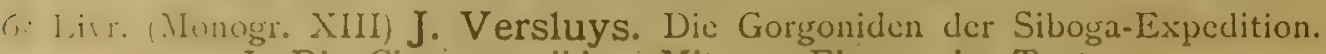

I. Dic Chrysogorgiidae. Mit I70 Figuren im Text.

7. Live (Monocr. XVIa) A. Alcock. Report on the Deep-Sea Madreporaria of the SibogaExpedition. With 5 plates.

Si Liv. (Mnnogr. XXV) C. Ph. Sluiter. Dic Sipunculiden und Echiuriden der Siboga-Exp. Mit 4 Tafeln und 3 Figuren im Text.

9. I.ivr. (Monngr. VIa) G. C. J. Vosmaer and J. H. Vernhout. The Porifera of the SibogaExpedition. I. The genus Placospongia. With 5 plates.

Io Livr. (Monogr. XI) Otto Maas. Die Scyphomedusen der Siboga-Expedition. Mit 2 Tafeln. I I Livr. (Monogr. XII) Fanny Moser. Die Ctenophoren der Siboga-Expedition. Mit 4 Tafeln. 12 Livr. (Monogr. XXXIV) P. Mayer. Die Caprellidae der Siboga-Expedition. Mit ro Tafeln.

I3" I.ir. (Afonomr. III) G. F. Tydeman. Hydrographic results of the Siboga-Lxpedition. With 24 charts and plans and 3 charts of depths

I f“ Lirr. (Monomi. NI,III) J. C. H. de Meijere. Dic Echinoidea der Siboga-Exp. Mit 23 Tafeln.

15" Livr. (Monomer. XI, Y a) René Koehler. Ophiures de l'Expédition du Siboga.

I Partie. Ophiures de Mer profonde. Avec 36 Planches.

\% 6.75 ๓9.-

$n 2 .-n 2.50$

"3.90 4.90

3., 3.75

$\pi 4.60,5.75$

"3.- $\times 3.75$

$72.40 \times 3 .-$

$" 7.50,9.50$

$72.80 \leadsto 3.50$

$7.80,9.75$

r 9.- $n 11.25$

"I 5.- ${ }^{2} \mathrm{IS} .75$

$\pi 16.50,20.50$

IG Liw. (Monogr. LII) J. J. Tesch. The Thecosomata and Gymnosomata of the SibogaExpedition. With 6 plates.

I $7^{2}$ Lirr. (Monogr. LVIa) C. Ph. Sluiter. Dic Tunicaten der Siboga-Expedition.

I. Abteilung. Die socialen und holosomen Ascidien. Mit I5 Tafeln

ISe Livr. (Monogr. LXI) A. Weber-van Bosse and M. Foslie. The Corallinaceae of the SibogaExpedition." With 16 plates and 34 textfigures

lge I.ivr. (Monogr. VIII) Sydney J. Hickson and Helen M. England. The Stylasterina of

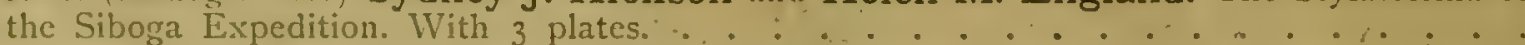

zoc Live (Monogr. XLVIIl) H. F. Nierstrasz. Die Chitonen der Siboga-Exp. Mit $\$$ Tafeln.

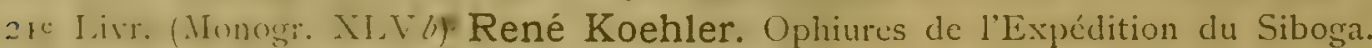

$2^{e}$ Partie. Ophiures littorales. Avec is Planches.

$\leadsto 3.75 \gg 4.70$

$6.75,9 .-$

$\eta 12.50 \cap 15.50$

$\rightarrow 1.50,1.90$

5. - 6.25

$710.25 \times 12.75$

$6.75,9 .-$

with an account of other species. With 14 plates and 2 text-figures.

$23^{c}$ Livr. (IIonogr. XXXVI) W. T. Calman. The Cumacea of the Siboga Expedition. With

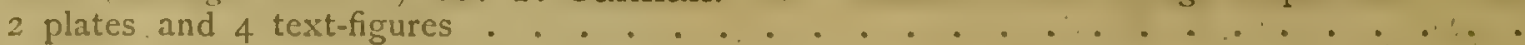

2.4c Livr. (Monogr. LVI $a$ ) C. Ph. Sluiter. Die Tunicaten der Siboga-Expedition.

Supplement zu der I. Abteilung. Dia socialen und holosomen Ascidien. Mit I Tafel.

25c Livr. (Monogr. L) Rud. Bergh. Die Opisthobranchiata der Siboga-Exped. Mit 20 Tafeln.

26c Livr. (Monogr. X) Otto Maas. Die Craspedoten Medusen der Siboga-Exp. Mit I4 Tafeln.

27 Livr. (Monogr. XIII a) J. Versluys. Die Gorgoniden der Siboga-Expedition.

II. Die Primnoidae. Mit 10 Tafeln, i78 Figuren im Text und einer Karte.

$1.80,-2.40$

$\rightarrow-75 \gg$ I.

๓11.25 „14.10

$\pi 9.25 \pi 12.50$

๓ $12.50,16.75$

2S: Lirr. Monogr. XXI) G. Herbert Fowler. The Chactognatha of the Siboga Expedition. With 3 plates and 6 charts.

Livr. Monosi. I.I) J. J. Tesch. Dic Ifteropoden der Siboga-Expedition. Mit I4 Tafeln.

Livr. (Monogr. XXX) G. W. Müller. Die Ostracoden der Siboga-Exped. Mit 9 Tafeln.

(IIonogr. IVhis) Franz Eilhard Schulze. Dic Xenophyophoren der Siboga-Exped. Mit 3 Tafeln

Mrmor. INI Maria Boissevain. The Scaphopoda of the Siboga Expedition. 6 plates and 39 textfigures

ThI J. W. Spengel. Studien uber dic Enteropneusten der Sibogr-Exp.

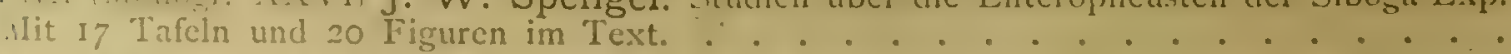

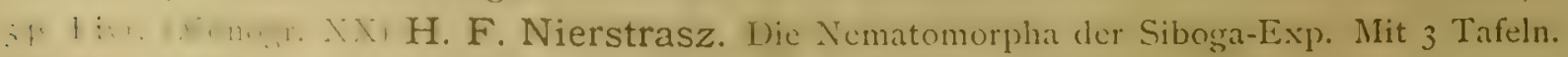
๓ $4.20 \pi .5 .25$

* $6.75 \approx 9 .-$

» $3.50,4.40$

ᄁ. $2.40 \quad 3 .-$

"4.80 $\leadsto 6 .-$

"14.- 17.50

$.2 .80,3.50$

Livr. (Monogr. XiII c) Sydney J. Hickson und J. Versluys. Die Alcyoniden der Siboga-

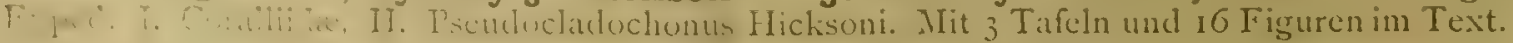

$\pi 2.20 \pi 2.75$ 






\section{(1)}

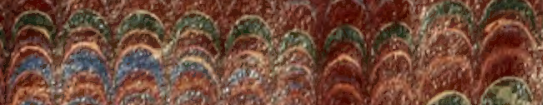

ahanân?

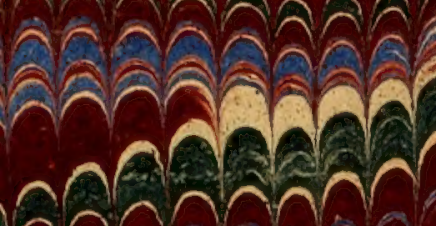

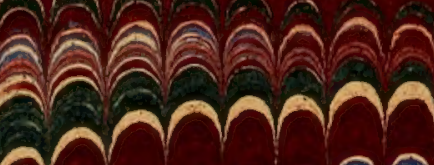

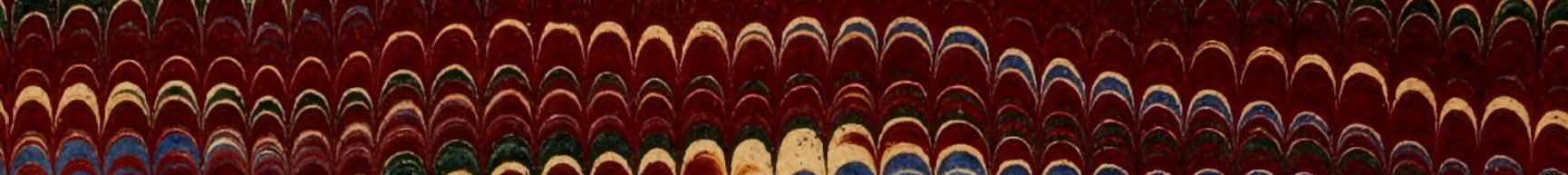

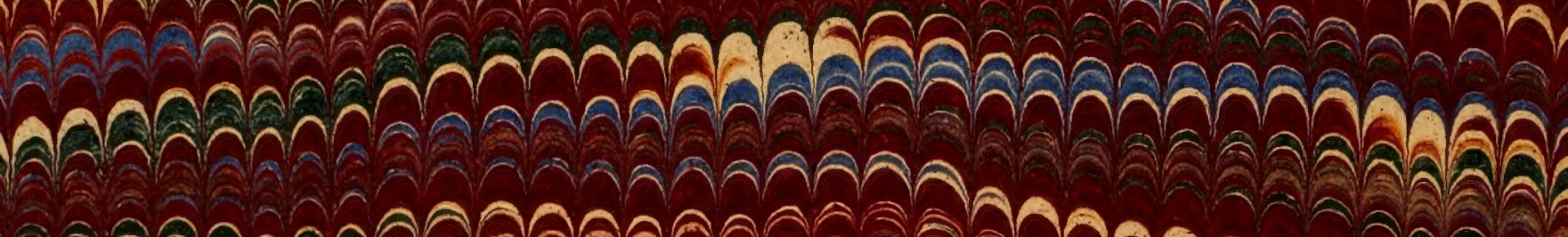

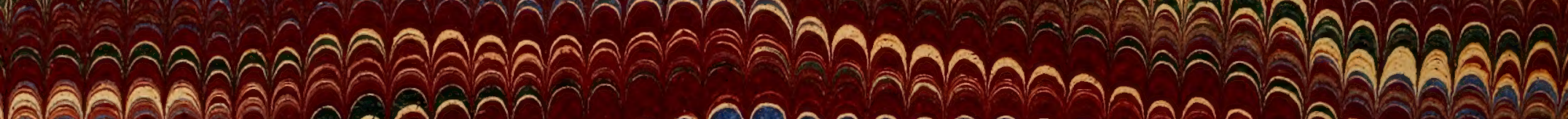

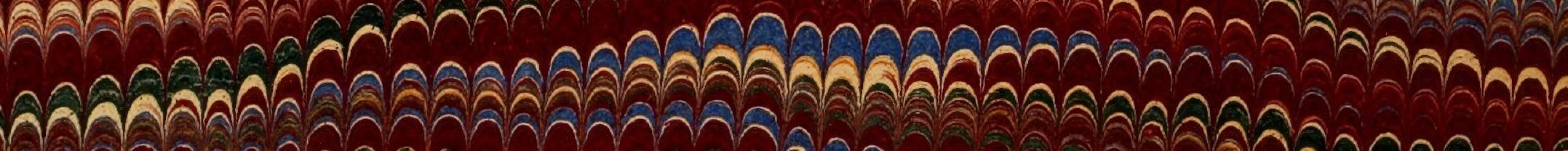

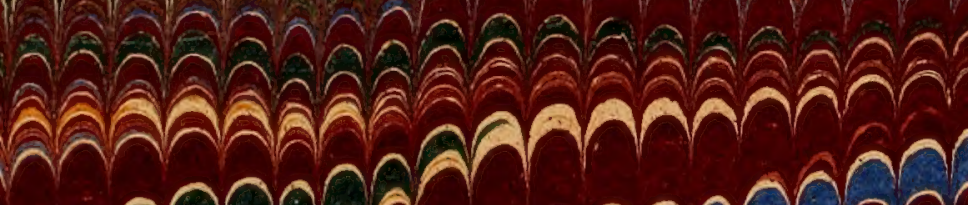

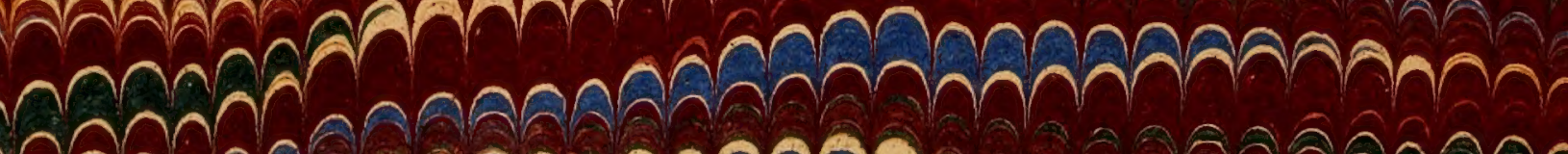

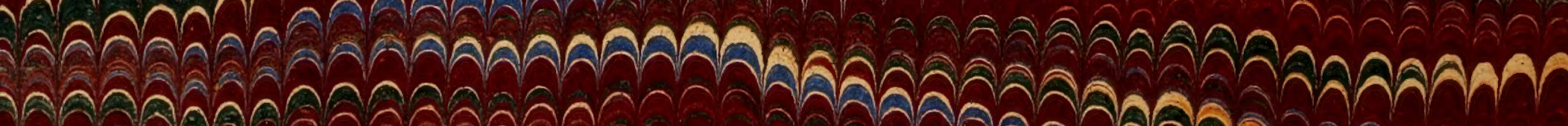

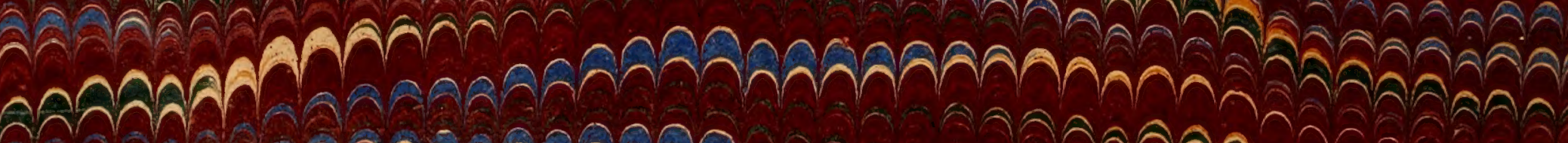

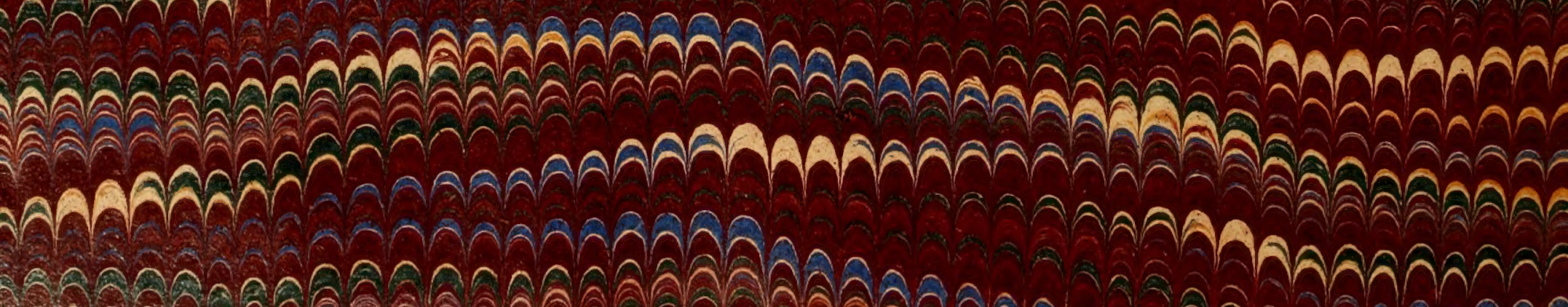
* - *a. 


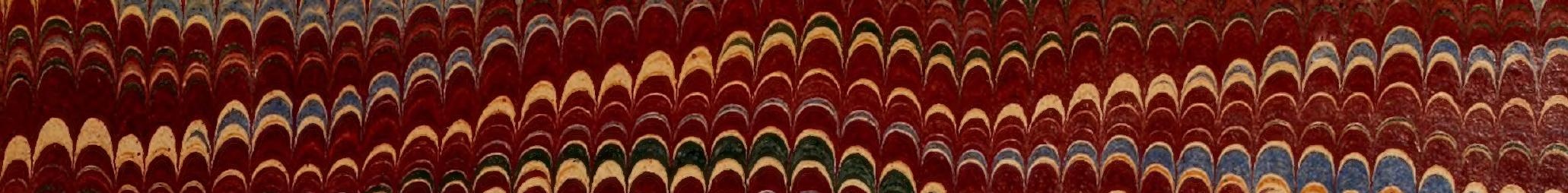

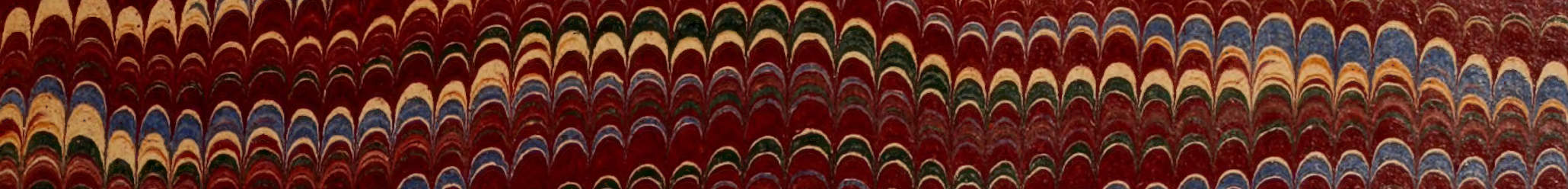

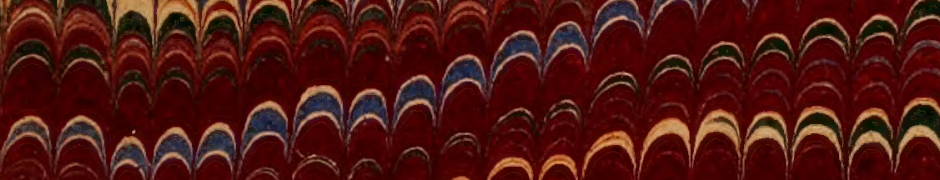

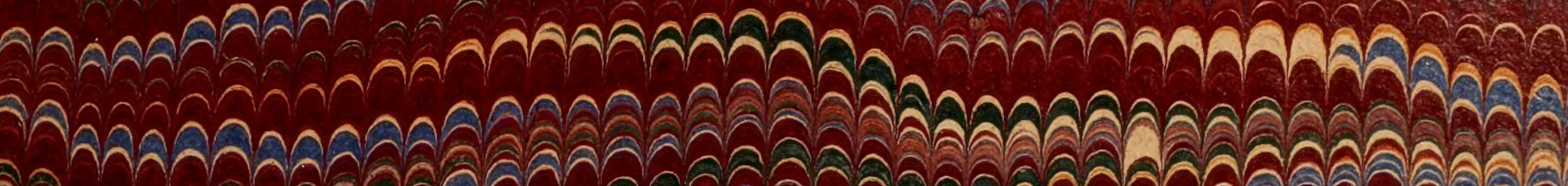

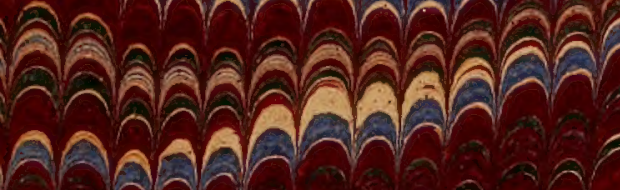

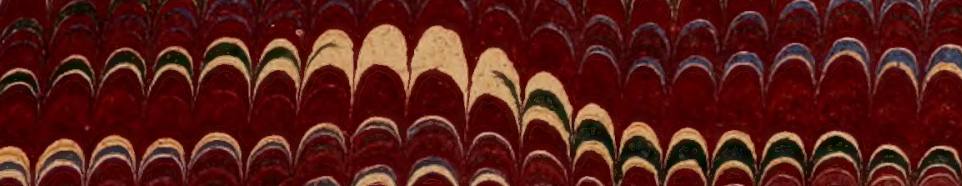
Arînaty TARAMAAARAAR

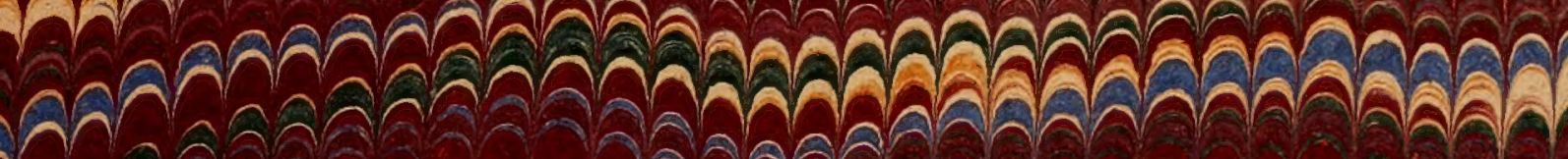
*añ

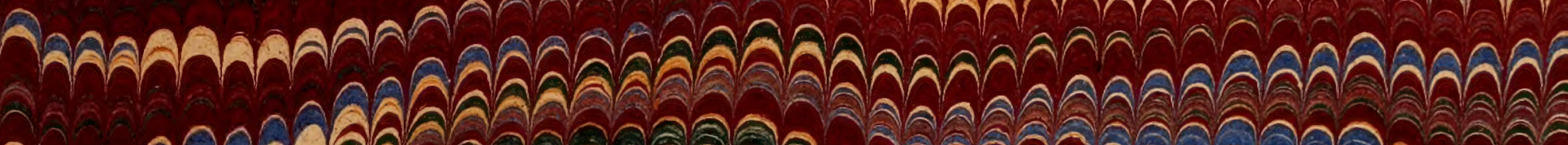

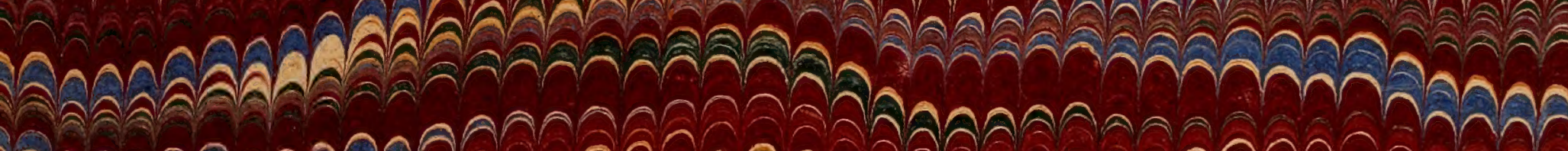
TaÂANA

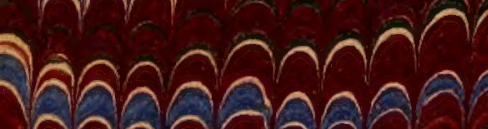
等

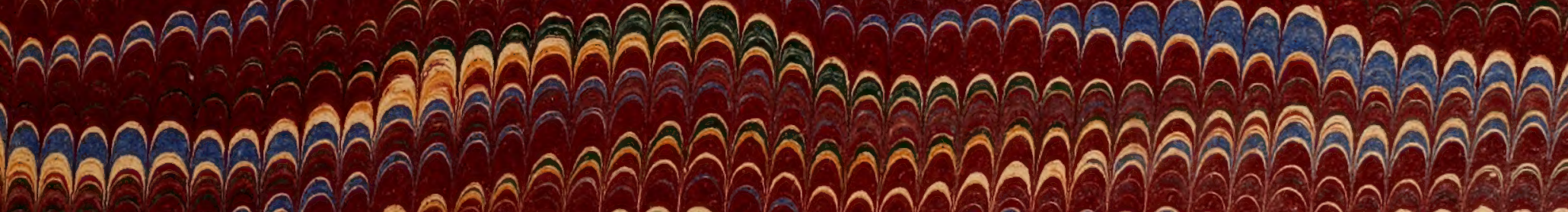

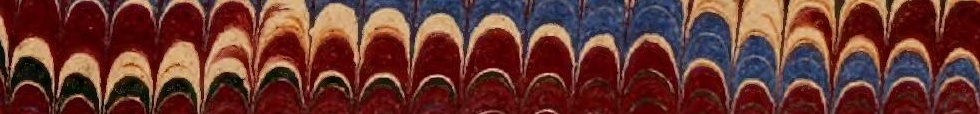

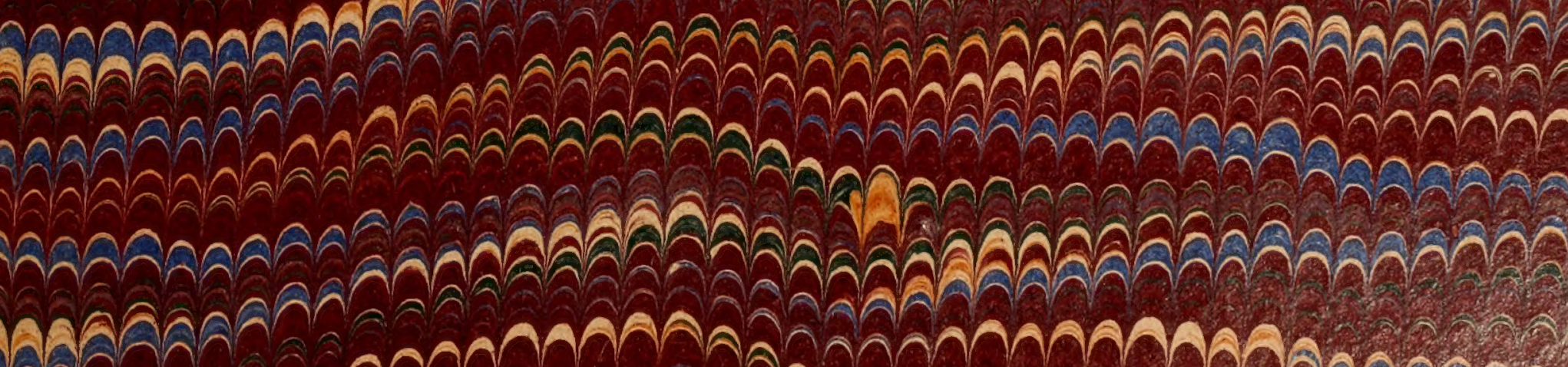

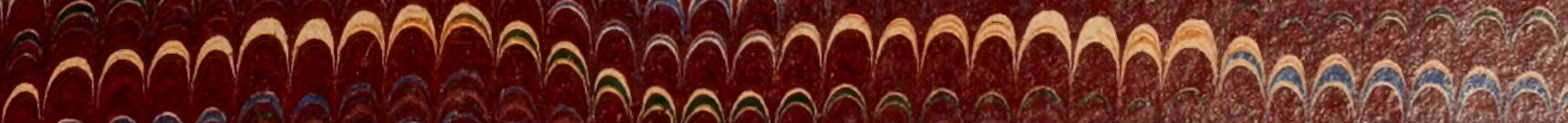

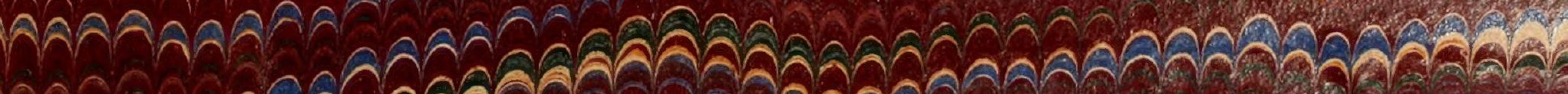




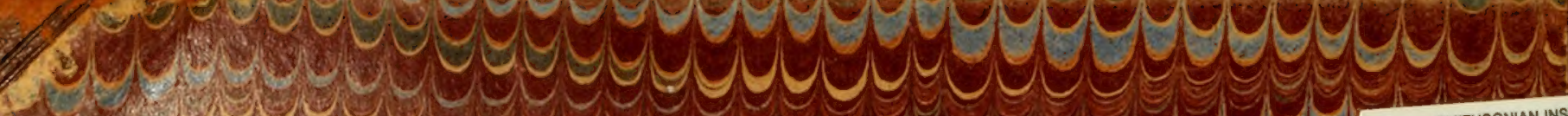
20.

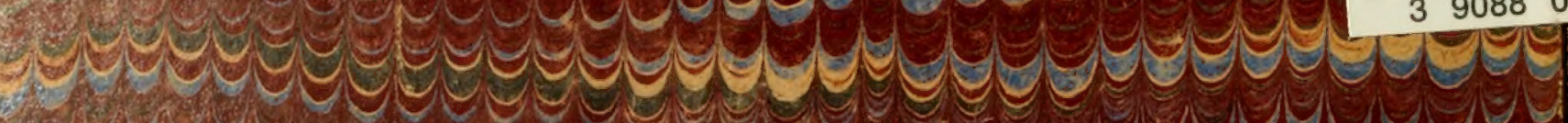

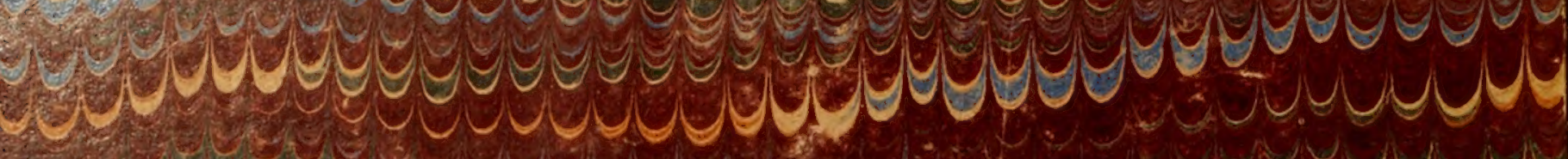
Cerar

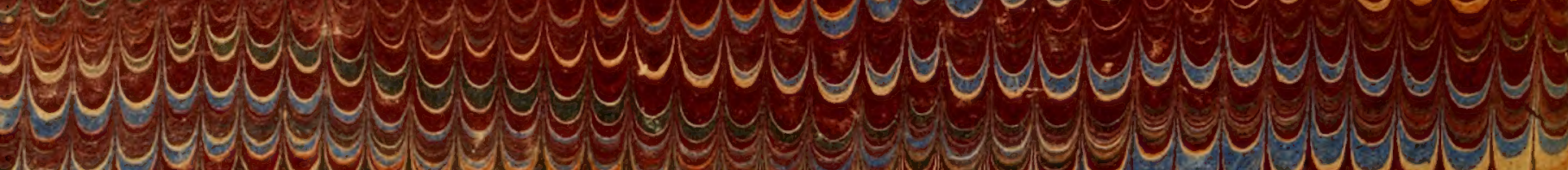

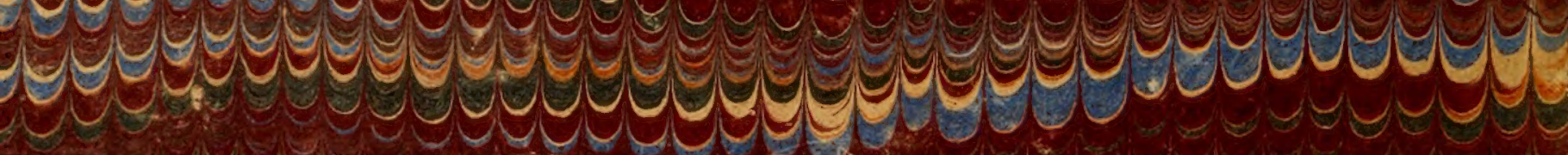

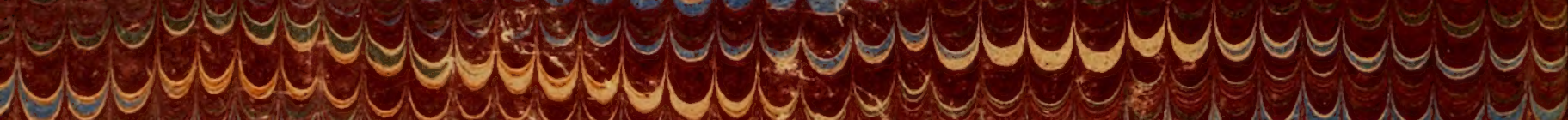
9 - _ yot a d a Y a c e c o

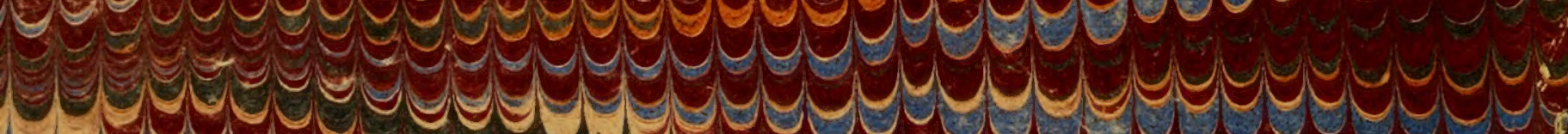

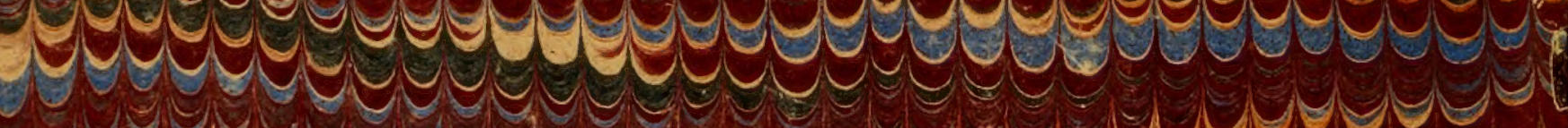

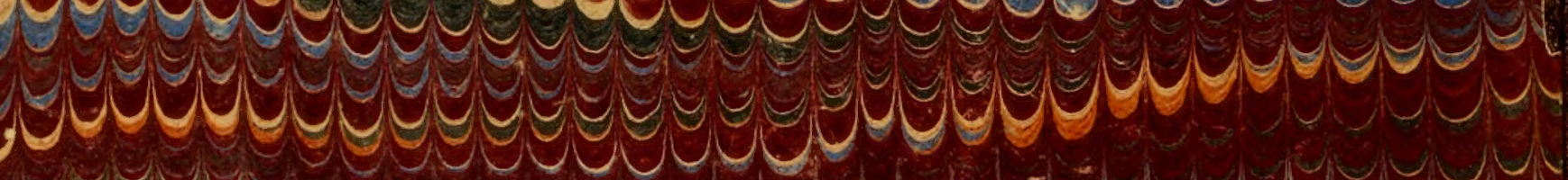

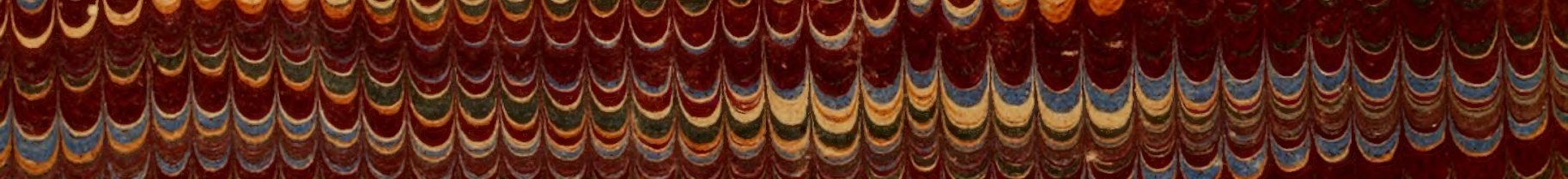
s yor. a

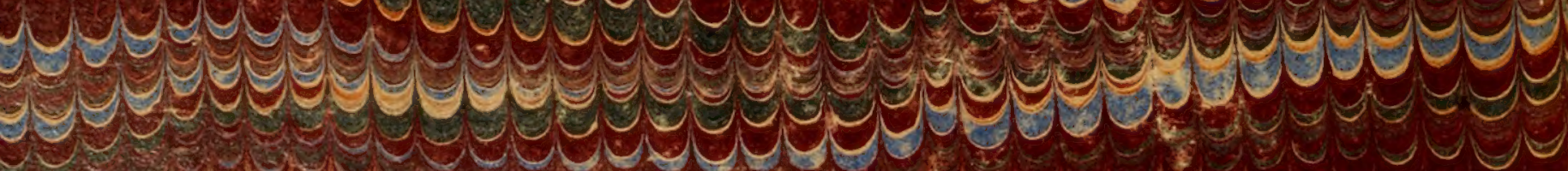

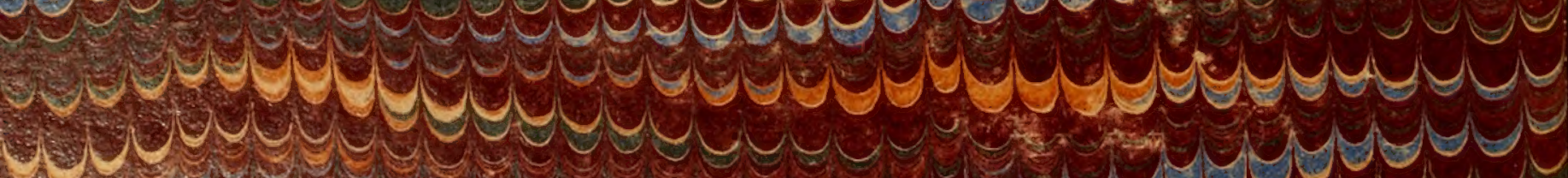
Y. S 4. 1. T-

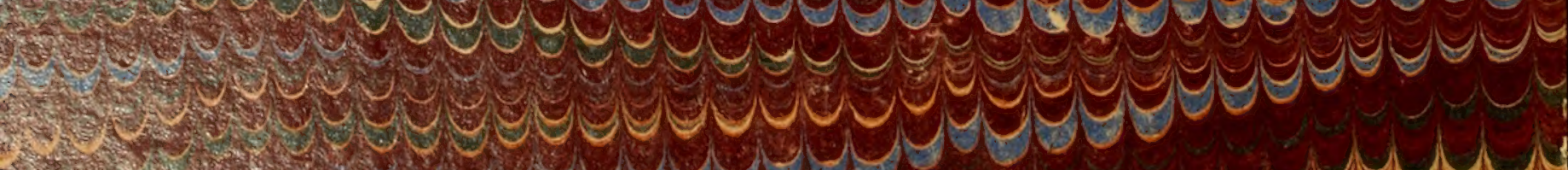

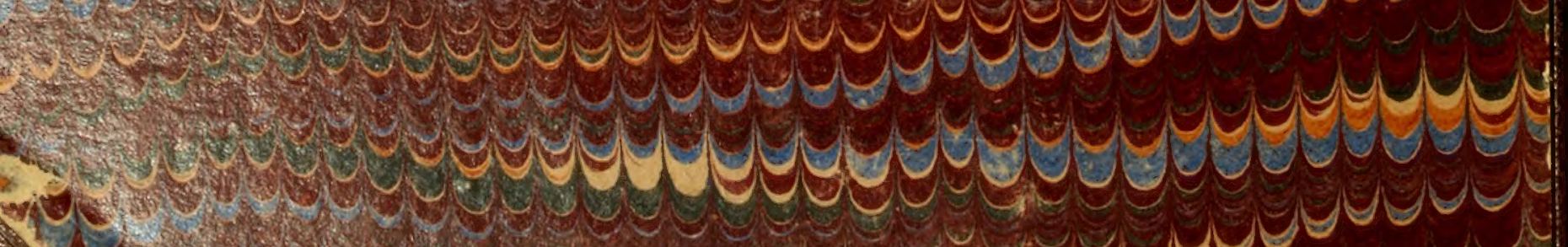

